\title{
RADIO-FREQUENCY SIZE EFFECT AND THE FERMI SURFACE OF ALUMINUM
}

John Sweeny Hartman

Ph. D. Thesis Submitted to Iowa State University

Ames, Laboratory, ERDA

Iowa State University

Ames, Iowa 50010

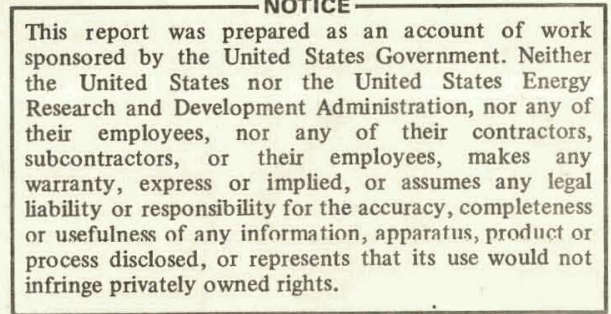

Date Transmitted: May 1975

PREPARED FOR THE U.S. ENERGY RESEARCH AND DEVELOPMENT

ADMINISTRATION UNDER CONTRACT NO. W-7405-eng-82 


\section{DISCLAIMER}

This report was prepared as an account of work sponsored by an agency of the United States Government. Neither the United States Government nor any agency Thereof, nor any of their employees, makes any warranty, express or implied, or assumes any legal liability or responsibility for the accuracy, completeness, or usefulness of any information, apparatus, product, or process disclosed, or represents that its use would not infringe privately owned rights. Reference herein to any specific commercial product, process, or service by trade name, trademark, manufacturer, or otherwise does not necessarily constitute or imply its endorsement, recommendation, or favoring by the United States Government or any agency thereof. The views and opinions of authors expressed herein do not necessarily state or reflect those of the United States Government or any agency thereof. 


\section{DISCLAIMER}

Portions of this document may be illegible in electronic image products. Images are produced from the best available original document. 
This report was prepared as an account of work sponsored by the United States Government. Neither the United States nor the United States Energy Research and Development Administration, nor any of their employees, nor any of their contractors, subcontractors, or their employees, makes any warranty, express or implied, or assumes any legal liability or responsibility for the accuracy, completeness, or usefulness of any information, apparatus, product or process disclosed, or represents that its use would not inf ringe privately owned rights.

Available from: National Technical Information Service U. S. Department of Commerce P.O. Box 1553 Springfield, VA 22161

Price: Microfiche $\$ 2.25$ 
Radio-frequency size effect and the Fermi surface of aluminum

by

John Sweeny Hartman

\author{
A Dissertation Submitted to the \\ Graduate Faculty in Partial Fulfillment of \\ The Requirements for the Degree of \\ DOCTOR OF PHILOSOPHY \\ Department: Physics \\ Major: Solid state Physics
}

Approved:

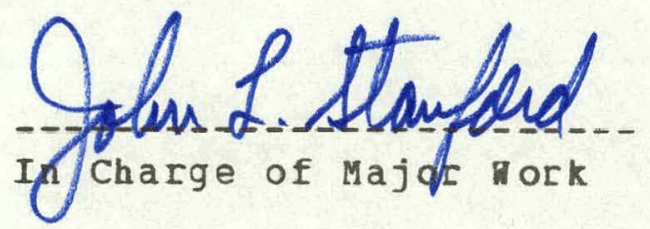

ROS Baring

For the Major Department

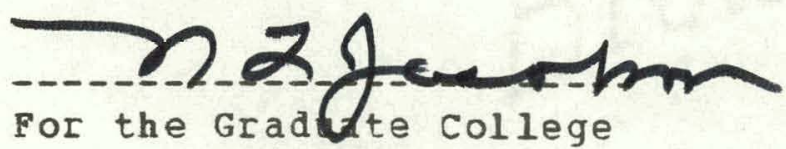

$$
\begin{gathered}
\text { Iowa State University } \\
\text { Ames Iowa }
\end{gathered}
$$

1975 
TABLE OF CONTENTS

\section{A.BSTRACT}

I. INT RODUCT ION

Page

II. THE FERMI SURFACE - . 2

A. The Basic Fermi Surface 2

B. The Permi surface of alaminum 4

III. THE RADIO-PREQUENCY SIZE EFPECT

A. The Radio-Frequencl Size Effect: Concepts 17

B. The Radio-frequency Size Effect:

F requency studies

IV. RADIO-FREQUENCY SIZE EFEECT SIGNALS IN ALOMINUM: COMPUTER PREDICTIONS 53

V: THE ALUMINOM EXPERIMENT

A. Apparatus $\quad 81$

B. Sample Holder assembly 92

C. Oscillator Problems $\quad 97$

D. Sample Preparation. 110

E. Sample Thickness 114.

VI. DATA AND DISCOSSION $\quad \begin{array}{ll}113 \\ \text { VIS }\end{array}$

A. Raw Angular Data 119

$\begin{array}{ll}\text { B. Frequency Studies } & 130\end{array}$

C. Fermi Surface zalipers and
orbit assignments

VII. CONCLOSIONS $\quad 175$

VIII. LITERATURE CITED

I X. ACKNOW LEDGIENTS 186 
Radio-frequency size effect and the. Fermi surface of aluminum

John Sweeny Hartman

Under the supervision of John L. Stanford

From the Department of Physics

Iova State oniversity

The radio-frequency size effect (RPSE) was used to determine Permi surface calipers in the (100), (110), and (111) planes for aluminam. The data vere recorded using frequency modulation detection and phase sensitive, techniques. The measurements were taken on flat, thin (100-180 microns) samples prepared from a large single crystal with a residual resistance ratio (RRR) of 13,000. Experimental angular data for each plane were compared with calipers taken from a computer calculation based on the nearly free electron (NFE) model of the second-zone hole surface for aluminum. The calculation yielded relative signal intensities expected for the second-zone signals from purely geometric considerations. These intensities were compared to the experinental results. Comparisons were also made with an adjusted NFE second-zone

1 USAEC Report IST-665. This rork was performed under Contract $-7405-e n g-82$ with the Atomic Energy Commission. 
model. wich included estimates of edge rounding based on previous stadies of aluminam.

The effects of non-uniform sample thickness on the frequency study technique for $f$ inding the appropriate RFSE magnetic field value were examined. Results of theoretical calculations were compared with data recorded in this investigation. Special consideration was given to the method proposed by cleveland for determining the frequency dependence of the BPSE line width and the resonant field value. It was found that the cleveland method gave accurate results only for perfectly flat samples. For samples with non-uniform. thickness, the resulting resonant field values were found to be lower than the appropriate value. This reduction was examined as a function of the thickness non-uniformity relative to the skin depth of the material. 
I. INTRODOCTION

Then undertaking an investigation of the electronic states (Fermi surface) of a metal many experimental techniques are available to the investigator. Methods such as the de Haas-van Alphen effect are well understood and the resulting data can be examined using strong mathematical tools. But the radio-frequency size effect is a relatively new experimental technique and several basic questions remain in interpreting the data.

This investigation was undertaken to use the radisfrequency size effect to exainine the well-known Fermi surfaze of aluminum. By choosing a well-known system the data analysis could also be directed at a better unjerstanding of the radio-frequency size effect itself.

This investigation was aimed specifizally at obtaining good mezsurements on tha Fermi surface of aluminum. It was also intended that the aluminum data be used to exanine the technique used for selecting the critical parameter from the recorded raw data. This parameter selection has let to uncertainties and discrepancies in past studies and must be evaluated if the radio-frequency size effect is to be utilized to maximum advantage. 


\section{FERMI SURFACE}

\section{A. The Basic Fermi Surface}

When the physical properties of a metal are examinad it is found that many of then (electrical resistance, specific heat, interaction with electromagnetic waves, etc.l depend at least in part on the behavior of tha conduction eleztrons in the metal. A concise and useful way to describe the states of the conduction eleztrons in the metal is to introduce the concept of a Permi surface (PS). Basic concepts behind Fermi surfaces will be discussed and then the FS of aluminum wil be examined specifically.

A single electron can be described by a position vector, $\bar{r}$, locating it in the crystal in raal space. Similarly it will have a momentum, $\bar{p}=\left(p_{x}, p_{y}, p_{z}\right)$, and it can be associated with the point $\left(p_{x}, p_{y}, p_{z}\right)$ in momentum space where the displacement along each axis is proportional to the particle's momentum in that jirection. For a classical particle and for free electrons the energy and momentum are related by Equation 1. The mass of the particle is represented by the symbol $\mathbb{m}$.

$$
E=p^{2} /(2 \text { m) }
$$

In the real orystal the electrons will ozcupy the lowest energy states compatible vith the pauli exelusion prineiple and the temperature of the sample. So for the free electron case where the energy is proportional to the square of the 
momentum, the states will be filled beginning at the origin of momentum space. As more electrons are assigned states. they will form spherical shells around the states already filled. For free electrons this leads to the states inside a sphere of radius $p_{F}$ being occupied and the states outside this sphere being empty. The momentum $p_{F}$ is salled the Fermi momentum. The surface dividing the occupied and unoccupied states is called the Fermi surface and is a constant energy surface. The energy on this surface is denoted by the symbol $E_{F}$ (the Fermi energy). For free electrons this pields Equation 2 .

$$
E_{F}=p_{F}^{2} /(2 m)
$$

As the temperature of the sample is increased, the sepzration between empty and occupied states becones less clear as the probability of occupancy for an electron state near the PS changes. Some states will be occupied above the FS at the expense of some states below the FS. The net effect of this is to cause the PS to become fuzzy rather than being a sharp dividing line between empty and occupiel states.

When real metals are examined, the periolicity of the crystal lattice and the interaction of conduction electrons with the fixed nuclei and localized electron zore states can greatly distort the free electron model of the FS. The lattice periodicity alone results in the introdustion of the concept of a Brillouin zone (BZ) and the assoiizted 
modifications in the FS as discussed by Kittel (1) (chapters 3 and 9 respectively). The FSs of most metals actually show little similarity to the free electron spherical FS.

Due to the smali mass and resulting small momenta of electrons in a metal, it is convenient to introduce the vavevector, $\bar{k}$, associated with an electron. The vaverector satisfies the following relations

$$
\begin{gathered}
h \bar{k}=\bar{p} \quad \text { or } \bar{k}=\bar{p} / \not \\
k=(2 \pi) / \lambda
\end{gathered}
$$

where $h=$ planck's constant (h) divided by $2 \pi$ and $\lambda$ is the de Broglie wavelength associated with the electron.

Equation 3 indicates the waverector is proportional to the momentum of the particle. Therefore the fS will be the same shape in $k$-space as it is in momentum space. It is standard practice to plot the FS in k-space. The conversion to k-space makes it much more convenient to deal with the numbers involved in describing the FS.

sany texts, including kittel $(1,2)$ and ziman (3), discuss in detail the additional factors that determine the actual shape of the FS for a given metal. They also discuss in detail how a knowledge of the FS can be used to calculate physical properties of the material.

B. The Permi Surface of Aluminum

The Fermi surface of a metal can be studied from tro different approaches. Theoretical calculations are carried 
out that include all the interactions between particles that the investigators feel are of importance for the metal. But the value of these calculations and their results cannot be evaluated without experimental evidence to substantiate or refute the results of the theoretical models. This exchange between theoretical results and experimental lata leads to $a$ better understanding of the physical processes that are actually important for determining the energy states of the conduction electrons in a metal. It also will lead to a better understanding of methods needed to solve difficult problems in the future.

Aluminum forms a face centered cubic (fes) lattice and the resulting $B Z$ in $k$-space is shown in Pigure 1. Due to the symmetry of the $\mathrm{BZ}$, information on $1 / 24$ of the entire zone is enough for a complete deszription of the zone and the FS. The portion of the $z$ one chosen is indicated on the figure and several points in the zone are labeled using the notation of Bouchaert et al. (4).

In 1957 Gunnarsen (5) used the de Haas-van Alphen (DHVA) effect to study aluminum. The DHVA effect yields information on cross sectional areas of the FS were external (maximum or minimum) cross sectional a reas occur (3, p. 274). Gunnersen's data at a given angle ere plottes on torque versus $(1 / H)$ plots. The curves showed oscillations that were periodic in $(1 / H)$. The periods of these oscillations are la- 


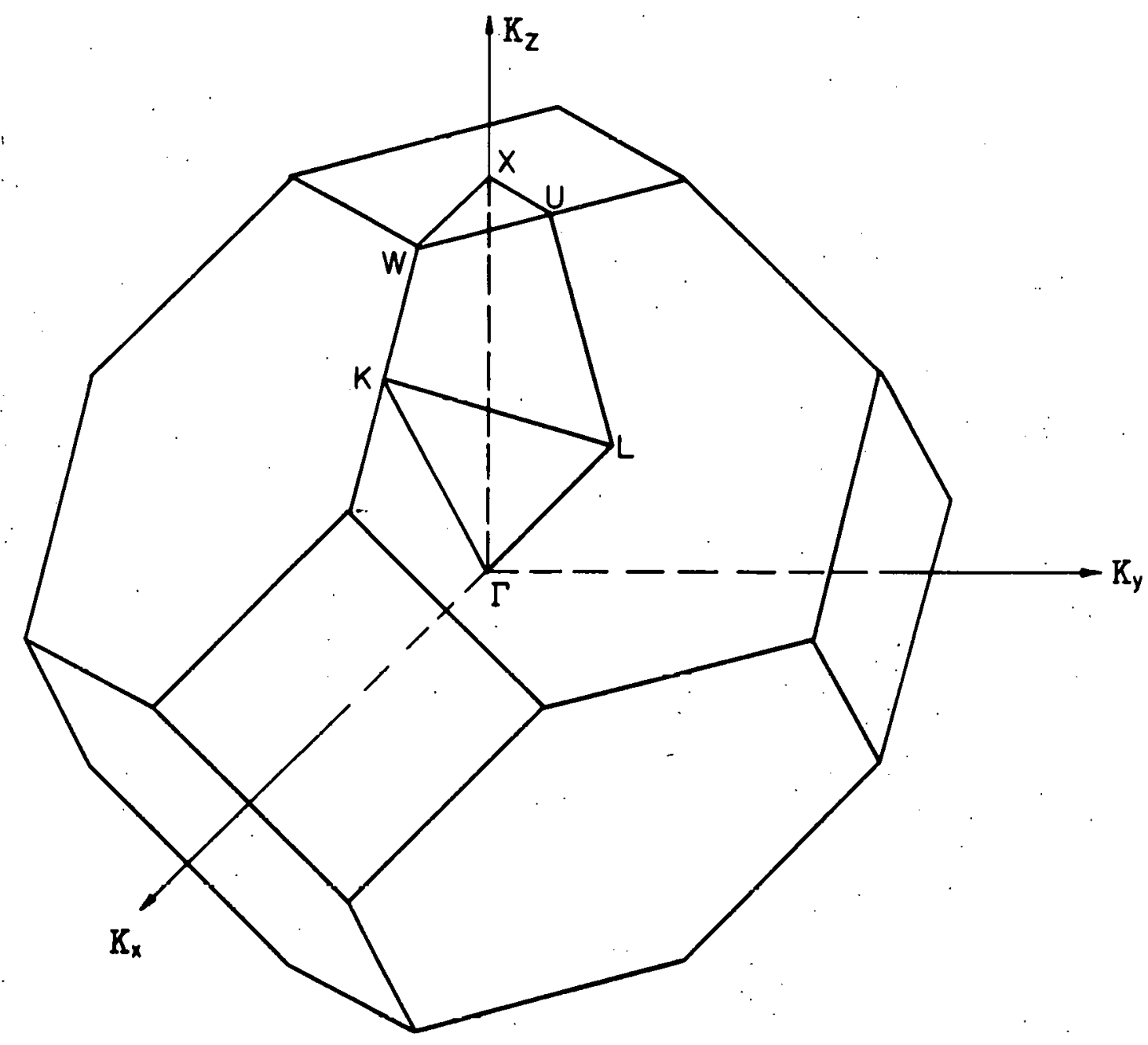

Figure 1. The Brillouin zone for a face centered cubic crystal structure with the important symmetry points labeled using the notation of Bouchaert et al. (4) 
beled $\Delta H$ and are related to the cross section area of the FS.

$$
\text { Period }=\Delta(1 / H)=(2 \pi e) /(\text { hCA })
$$

In Equation 5, A represents the extremal area of the FS giving rise to the signal. Heine (6) noted that the angular dependence of the data can be used to deduce the shape of the cross section only if the cross section results from a centrosymmetric piece of the FS. If the $\approx$ ross, section belongs to a non-centrosymmetric piece of the PS then we can only deduce a symetrical surface which has the same angular dependence for the area as the actual fS cross section but the deduced shape need not be correct.

Gunnersen obsecved signals in two distinzt frequency ranges that differed by an order of magnitude. These were referred to as high and lov frequency signals. Some authors have referred to them as short period and long period data respectively. Gannersen associated the high frequency oscillations with pockets of positive holes located at $\omega$ in the first BZ. This assignment followed his examination of Heine's (6) calculation on the FS of aluminum. Gunnerson's low frequency data were not of sufficient quality or quantity to allow determination of angular dependence of the FS area or an assignment to a sperific region of the PS. A study employing several alloys did lead to the conclusion that these data resulted from an electron surface. 
Heine $(6,7,8)$ carried out an orthogonalized-plane-wave (OPR) calculation and examiaed the resulting PS in light of Gunnersen's DHVA data and earlier aluminum data taken in studies of the anomalous skin effect (9) and the lowtemperature specific heat $(10)$. Heine found the shape of the FS away from the zone boundaries to be very siailar to the free electron model. The FS model resulting from this examination had portions of the first three zones occupied. The first zone was completely occupied by electrons except for small regions of holes around the corners of the $B 2$ at 1. The second zone consisted of fourteen spherical caps, one on each of the BZ faces. The third zone consisted of small electron surfaces near the corners. Heine's calculation and construction represented the first extensive effort to combine experimental data with energy band calculations to arrive at a FS nodel.

Fawcett (11) and Langen berg and noore (12) carried out cyclotron resonance (CR) studies of aluminum and found their results compatible with the theoretical calculations of Heine (6, 7, 8). Two distinct groups of carriers were indicated in each study and Langenberg et al. associated their low mass signals with the holes that Heine had placed at the corners of the first zone.

Harrison (13) then re-examined Gunnersen's data and found that only slight modifications of the nearly free elec- 
tron. (NPE) FS led to excellent agreement. The calculation began with a construction of the NFE PS by the method that is now known as Harrison's construction. The resulting model is seen in Figure 2. The first zone is full. The second zone contains a single hole surface. The third anj fourth zones contain regions of electrons. The NFE model was examined to determine if anticipatel DHVA signals would show the symetry seen in Gunnersen's data. Modifications were made were nezessary to arrive at areas compatible with the angular DHVA data. The modifications needed for general agreement occurred in the third and fourth zones. The electron surfaces in the fourth zone were deleted from the model. Tha shape of the third zone arms was changed but they maintained their original connectivity. The shape Harrison found compatible with the DHva data is shoun in Figure $3 a$. It was also noted that the sharp edges on the second zone hole surface would be rounded off but no attempt was made to determine how great the rounding would be. The previous interpretation of low mass CR signals would be in error ander Harrison's model. In 1960 Harrison (14) carried out further calculations on the energy bands and the resulting FS using a pseudopotential method. FS cross sections vere obtained for several planes passed through the BZ. The cross seotions found indicated the rounding expected at the sharp points of the NFE model of the FS dae to interactions omitted from the 

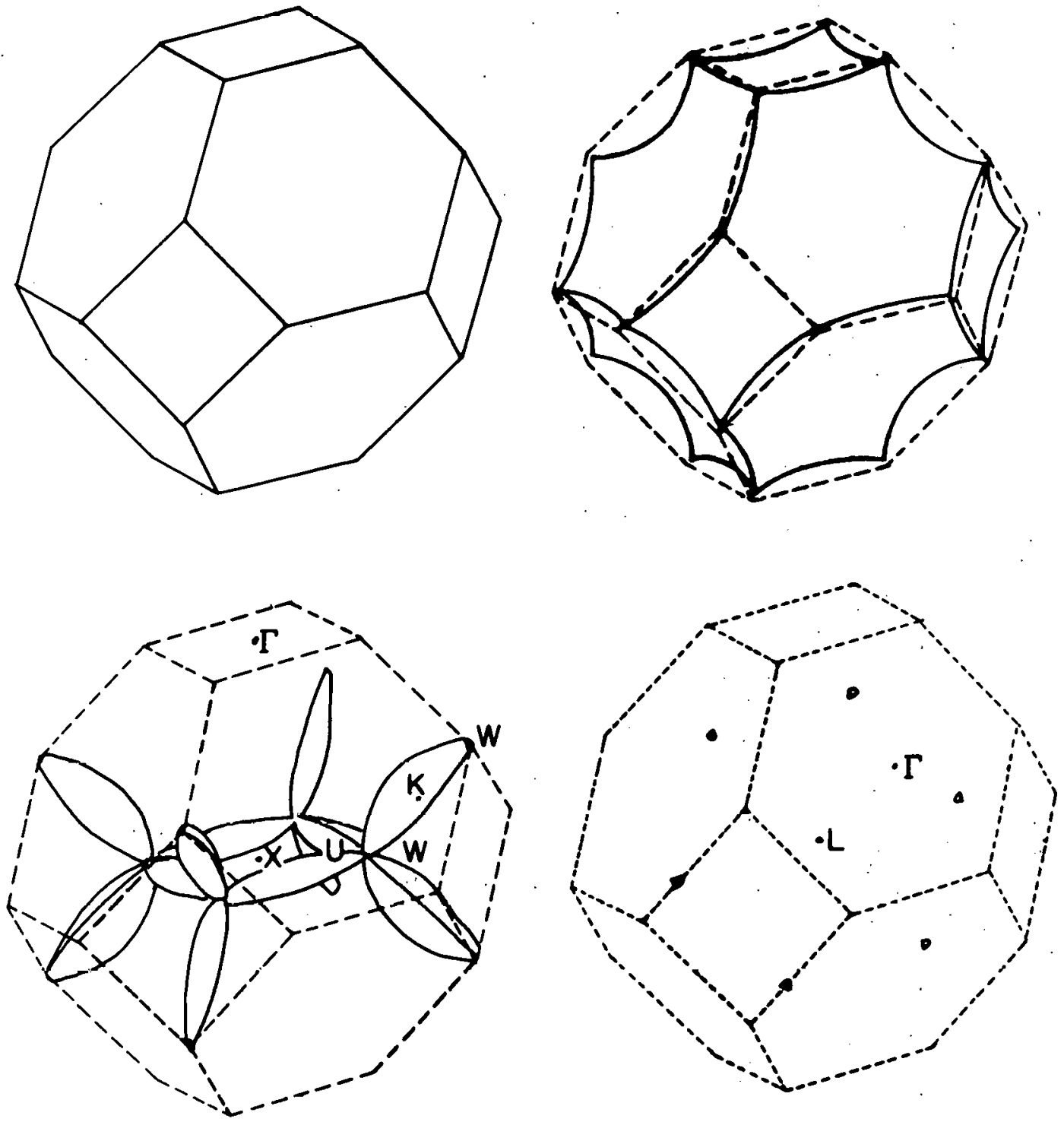

Figure 2. The NFE Fermi surface of aluminum after Harcison (13) showing the first, second, third, and fourth zones 

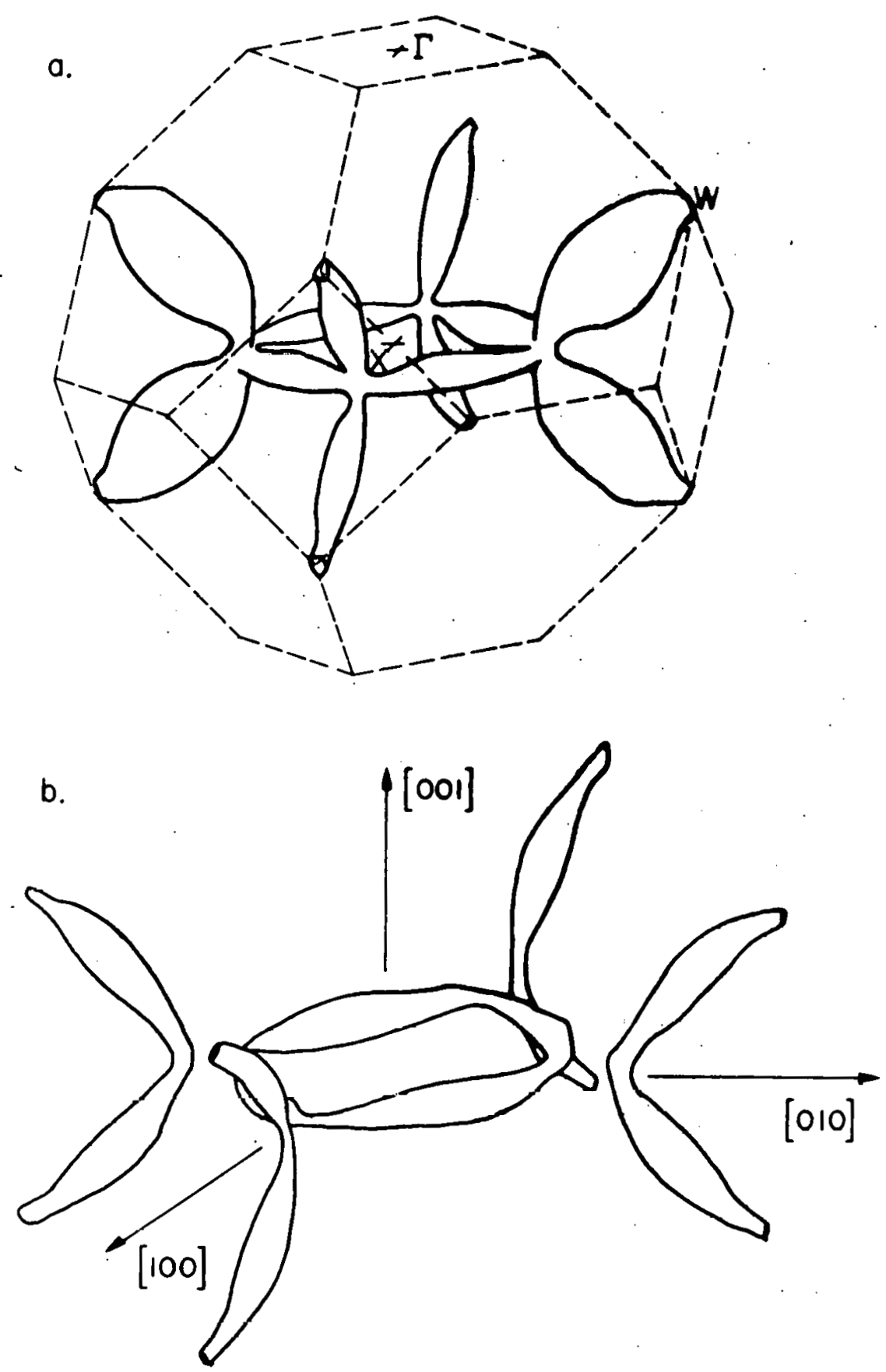

Figure 3. Third zone electron arms of the aluminum Fermi surface after a) Harrison (13) and b) Ashcroft (18) 
NFE calculation. The cross section shown for a plane containing one of the square faces at $X$ in the $B Z$ indicated the sensitivity of the third zone to small changes in the Fermi energy. Using the permi energy found in a single oph calculation, the third zone mojel was compatible with the earlier model shoun in Pigure $3 z$. But small changes $( \pm 2 \pi)$ in the Fermi energy would cause the arms to swell or be pinched off into small groups of isolated electrons centered at $k$ in the BZ. Due to the sensitirity of the resultant FS at $W$ Harrison could make no definite statement regarding the exazt form of the third zone PS based on this calculation. But using the single $O P W$ value of $E_{F}$ the model was in agreement with areas previously discussed from DHVA data. The model also gave semiquantitative agreement with CR jata of Fawcett (11). Moore and Spong (15), and Langenberg and Moore (12) with their low mass data now being attributed to orbits around the third zone arms.

segal (16) carried out an energy band calculation using the Green's function method. His results vera in basic agreement with Harrison's model but he noted that the semiquantitative agreement between the CR effective mass data and the model prediction should not be disturbing. He pointed out that actual masses will depend on electron-electron and electron-phonon interactions which have not been included in calculating the nasses from the PS model. It is believel 
that these effects would be appreciable and would correct the theoretical value in the jirection needed to agree with experimental data.

In 1962 Priestley (17) used the DHVA technique to examine aluminum. An effort was made to find very bigh frequency signals that would correspond to the second zone hole surface. No attempt was made to reproduce the lower frequency data of Gunnersen (5) and interpreted by Harrison (13). Signals were observed that a greed well with the model of the second zone piece. Also, signals vere seen due to electrons moving around the inside of the four arms on a square face. Thus the data gave good quantitative agreament with the NPE third zone model of Harrison.

The main question remaining after this calculation was the connectivity of the third zone arms. Harrison noted that the accuracy of his 1960 calculation was not sufficient to make a definite statement concerning the form of the third zone arms near

In 1963 the DHVA data of Gunnersen (5) and Priestley (17) were re-examined by Ashcroft (18) in the development of a ner phenomenological model for the PS. The resulting model showed one major difference from the NFE model. Ashcroft proposed that the third zone arms were connected in groups of four around each square face on the Bz but that adjacent groups of arms were not in contact at point $W$ of the $B Z$. The 
proposed third zone model is shown in Figure 3 b. Recall that point $W$ is precisely here Barrison (14) could make no definite statement about the fS connectivity iue to the extreme sensitivity of that region in his calaulation. In 1967 a definitive experiment for the third zone connectivity was performed by Larson and Gordon (19) using the DHVA method. These data were found to be in excellent agreement with Ashcroft's model.

Aditional theoretical and experimental results have been reported but they have not altered the model of the BS appreciably. Mang of these works are indicated below but they will not be discussed. Some of these works will be referred to when the data from this present experiment are examined in a later section. Articles marked with an asterisk (*) have already been cited in this work.

Energy band and FS calculations

*Heine $(6,7,8)$

* Harrison $(13,14)$

Harrison (20)

* Segal $(16,21)$

*Ashcroft (18)

S now (22)

Greizen (23) (Correction to snow's result.)

Connolly (24)

Faulkner (25) 
Hoffstein and Boudreaux (26)

de Haas-van Alphen experiments

* Gunnersen (5)

* Priestley (17)

* Larson and Gordon (19).

Anderson and Lane (27)

Shepherd et al. (28)

Cyclotron Resonance experiments

* Langenberg and Moore (12)

* Fawcett (1.1)

* Moore and spong (15)

Galkin et al. (29)

Naberazhnykh and Tolstoluzhskii (30)

Spong and Kip (31)

Magnetoacoustic effect experiments

Roberts (32)

Bezuglyi et al. (33)

Jones (34)

Fossheim and olsen (35)

Kamm and Bohm (36)

Kohn anomaly experiments

Stedman and Nilsson (37)

heymouth and stedman (38)

Galvanomagnetic effect experiments

Balcon be (39) 


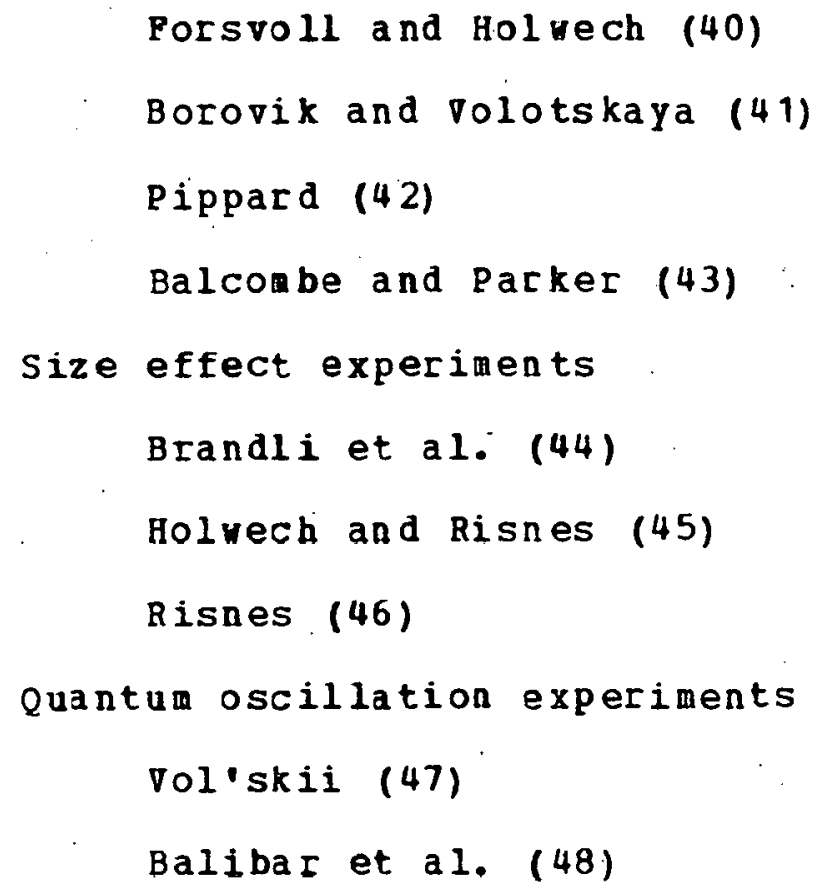




\section{THE RADIO-FREQUENCY SIZE EFFECT}

A. The Radio-Frequency Size Effezt: Concepts

The RFSE is based on the study of anomalies in the surface impedance of single crystal, thin, flat, metal samples in a static magnetic field applied parallel to the surface of the sample. The surface impedance is examined by studying the interaction of the sample with radio-frequency (rf) radiation incident on the sample's surfaze. Anomalies in the surface impedance are observed for magnetic field values that cause electrons executing cyclotron motion to span the sample thickness and pass within the skin depth on each surface of the sample (49). For high purity metals at low temperatures, the mean free path of the electrons should be long enough to allow the particle to visit both surfaces before it is scattered. For samples not meeting this requirement, the RFSE is not observal. It will be shown that the magnetic field values corresponding to anomalies in the surface impedance can be related directly to FS dimensions. The RFSE was used in this capacity to examine the FS of aluminum in this investigation.

Throughout the remainder of this work the motion of electrons in real space and in $k$-space will be examined. Several terms should be explained now to avoid possible confusion in the remainder of the text. The path followed by a particle in real space uill be referred to as the parti-. 
cle's real space trajectory or simply the trajectory. The particle's motion in $k$-space will be called a k-space orbit or simply an orbit.

As previously stated, the anomalies in the surface impedance are observed wen the magnetic field value causes cyclotron trajectories to span the sample thickness. In this situation the electron spends time in the skin depth on both sides of the sample and is able to absorb pover from the rf fields in each skin depth. The frequency of the incident. If field is chosen to be much less than the cyclotron frequency. This means that for electrons making more than one cyclotron rotation before scattering, they experience essentially the same field each time they return to the skin depth. Therefore, multiple passes through the skin depth enhance the power absorbed and the electron's effect on the surface impedance anomaly. This effect leads to stronger signals as the mean free paths of the electrons incraase. Since the frequencies of the rf field and the electron rotation are not matched, this is not a temporal resonance as in the case of cyclotron resonance. Rather it is the size of the trajectories and the thickness of the sample that are matchad so the RFSE is actually caused by a spatial resonanze.

The surface lapedance anomaly resulting from the cyclotron motion spanning the sample can be related to Fermi surface dimensions by considering an electron's motion in 
both $k$-space and real space. Begin by examining the motion in $k$-space. The electron is acted on by the standard Lorentz force,

$$
\overline{\mathrm{F}}=d \overline{\mathrm{p}} / \mathrm{d} t=\hbar \mathrm{d} \overline{\mathrm{k}} / \mathrm{d} t=(e / c)(\bar{v} \times \overline{\mathrm{B}})=(\mathrm{e} / \mathrm{c})(\mathrm{d} \overline{\mathrm{I}} / \mathrm{d} t \times \overline{\mathrm{B}})
$$

where e=charge of the electron, c=speed of light, $\bar{v}=v e l o c i t y$ of the electron, $\overline{\mathrm{H}}=$ the applied magnetic field, and $\bar{r}=$ position vector locating the electron in the crystal. The force is always perpendicular to the velocity and the magnetic field. The first fact results in the electron's energy remaining constant and the second results in the conponent of momentum parallel to $\bar{H}, k_{H}$. remaining constant. In k-space, this restricts the particle's motion to the FS (a constant energy surface) and to a plane perpendicular to the applied magnetic field.

The particle will move around its orbit at the cyclotron Erequency.

$$
w_{c}=e H / m * c
$$

where $m *=e f f e c t i v e$ mass of $t$ he electron.

Integrating Equation 6 with respect to time leads to Equation 8 .

$$
\hbar \Delta \bar{k}=(e / c)(\Delta \bar{r} \times \bar{H})
$$

From this it is seen that the electron's orbit in k-space will be the same shape as the projection of the real space trajectory on a plane perpendicular to the applied nagnetic field, but it will be rotated by $90^{\circ}$ about the direction of 
$\overline{\mathrm{H}}$. The projection of the trajectory must be examined because keeping $k_{H}$ constant in $k$-space still allows motion parallel to $\overline{\mathrm{H}}$ in real space. Therefore, in real space the trajectory may be confined to a plane or it may have a helical shape. It is also seen from Equation 8 that for a given value of $|\Delta \bar{k}|$, the magnitude of $|\Delta \bar{I}|$ is inversely proportional to the magnitude of the magnetic field. Therefore, as the magnetic field is increased, the projection of the trajectory decreases in size.

In the RFSE, the size limitation placed on the real space orbits is in the direction of the sample normal, $\bar{n}$, where the sample thickness vill be represented as $t$. In Equation 8 we can then write $\Delta \bar{r}=t \bar{n}$. Hith $\bar{n}$ and $\bar{H}$ in the previously assigned directions, $\Delta \bar{k}$ wst then be parallel to the sample's surface and perpendicular to $\overline{\mathrm{H}}$. The magnitude of $|\Delta \bar{k}|$ will then be

$$
|\Delta \bar{k}|=(e / h c) t H
$$

where $|\Delta \bar{k}|$ now represents the FS caliper in a direction perpendicular to $\bar{n}$ and $\overline{\mathrm{H}}$ for the electron orbit under consideration. It is easily seen from this that an accurate determination of the sample thickness and the magnetic field value for the surface impedance anomaly can yield accurate FS calipers. Figure 4 illustrates the relationships between real and $k$-space orbits and the FS caliper determined by such a measurement. Consider a light beam aimed down the normal 
a) $\quad \kappa$-SPACE

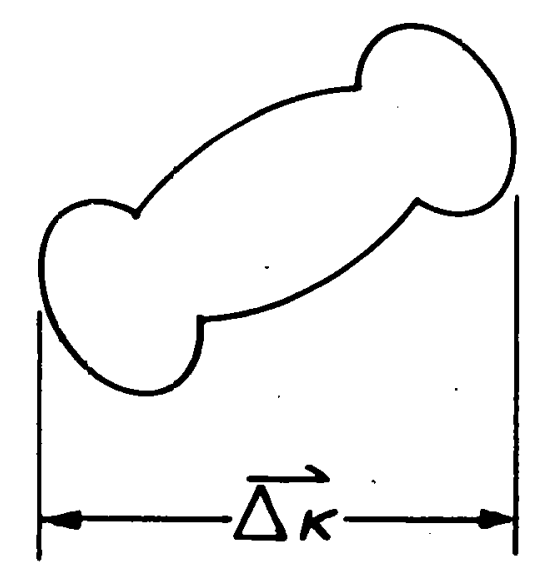

"b) REAL SPACE

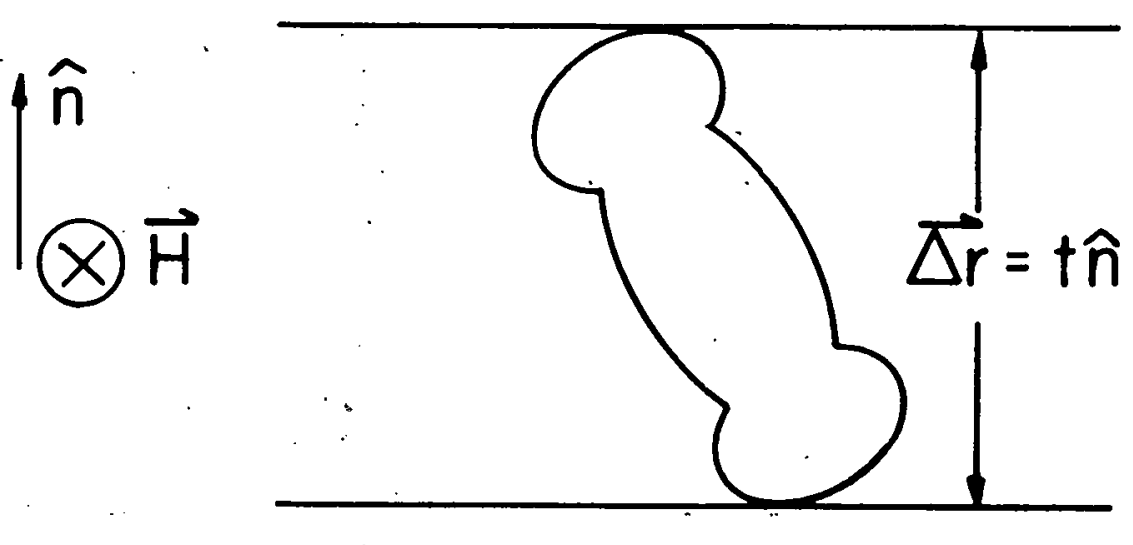


direction, $\bar{n}$, toward the FS. It is seen that $|\Delta \bar{k}|$

corresponds to the length of the FS shador on the line common to the plane perpendicular to $\bar{n}$ and the plane perpendicular to $\bar{H}$. For this reason it is posisible to take a sample with $z$ [klm] normal axis and use the RFSE to measure the calipers of the FS shadow on the (klm) plane. As the direction of $\bar{H}$ is changed in the plane perpendicular to $\bar{n}$, the zalipers for the entire shadow can be measurad. It is seen that $\Delta \bar{k}$ may not correspond to a FS dimension in a plane parallel to the sample surface if the uidest points on the FS lio in different planes perpendicular to $\bar{n}$. This possibility must be kept in mind when data are interpreted.

The use of an If field incident on the surface of the sample provides a very important contribution by causing the surface impedance anomaly to be relatively narrow. As stated earlier, in order to make a significant contribution to the signal the electron should spend some time in each skin depth. But this can occur for a variety of raal space trajectory diameters as illustrated in Figure 5. This illustration is based on a circular electron trajectory to simplify the equations. For a circular orbit, the magnetic field $H$ which leads to a trajectory of diameter $D$ is given by $H=(2 m v c) /(e D)$

where vihe electron's relocity. For the example in the figure this leads to 


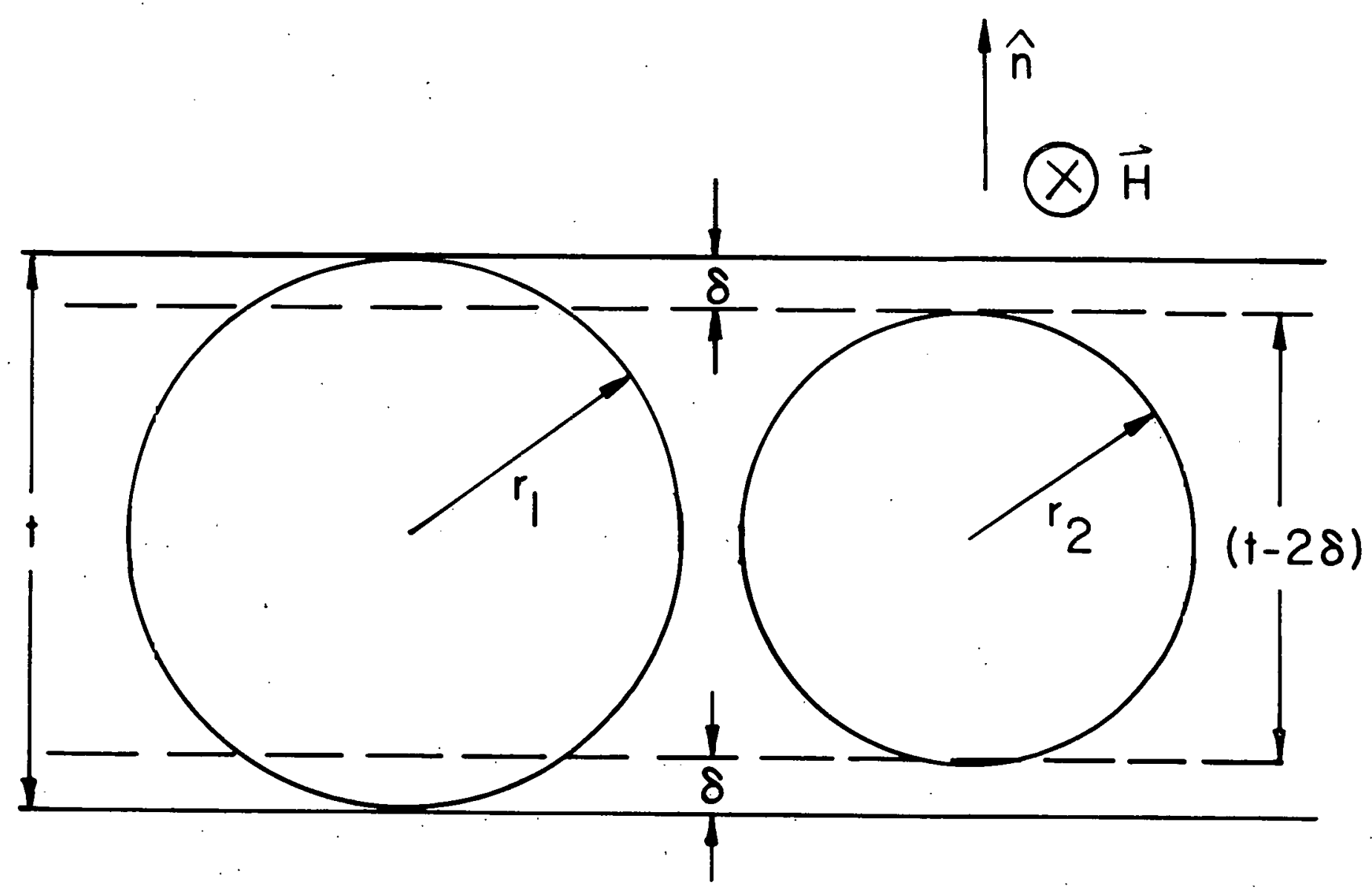

Figure 5. Field broadening of the RFSE resonance results from the penetration of rf fields into the skin depth. Tha real space trajectories shown illustrate the limits of trajectories contributing to the RFSE resonance 


$$
\mathrm{H}_{1}=(2 \mathrm{~m} \nabla c) /(\mathrm{et}) \text { and } \mathrm{H}_{2}=(2 \mathrm{mvc}) / \mathrm{e}(t-2 \delta)
$$

where $\mathrm{H}_{1}$ will be smaller than $\mathrm{H}_{2}$. Consider increasing the magnetic field from a value below $H_{1}$. Mhen $H_{1}$ is reached the anomaly in the surface impedance will appear as it is now possible for a complete cyclotron trajectory to occur with the electron visiting both skin depths. As the field is increased further, this trajectory continues to contribute to the anomaly until it is no longer possible for the electrons to enter the skin depth at each surface. This termination of the anomaly occurs at $\mathrm{H}_{2}$. Thus the anomaly is spread over 1 range of magnetic field $\Delta H$ given by

$$
\Delta \mathrm{H}=\mathrm{H}_{2}-\mathrm{H}_{1} \text {. }
$$

The fractional width of the signal can be expressed as $\left(\Delta H / H_{1}\right)$ where $H_{1}$ corresponds to the actual onset of the a noma1y.

$$
(\Delta H) / H_{1} \cong(2 \delta) / t
$$

From this it is easily seen that the anomaly zan be sharpened by decreasing the skin depth wich can be done by increasing the frequency of the incident if radiation. For good samples. at low temperatures this can lead to a fractional width of the signals of from one to five per cent.

The idea of sharpening the anomaly by increasing the Erequency was first noted by Krylov and Gantmakher (50). It is seen from Equation 11 that the low field onset of the anomaly does not wove as the skin depth decreases but all 
other features of the anomaly signal will converge toward $H_{1}$ as $(\Delta H / H$,$) decreases. This narroying of the anomaly has been$ utilized on several occasions to determine the field at the onset of the resonance condition, $H_{f}$, and is referred to as the frequency study technique.

To carry out a frequency study, RPSE data is taken at several frequencies for the same surface impedance anomaly. In this investigation the data will be plotted on an $X-Y$ recorder where the vertical axis represents the derivative of the surface reactance with respect to the magnetic field and the horizontal axis represents the value of the magnetic field. Figure 6 a shous a typical trace from zleveland's study of molybdenum. The individual features of the signal are labeled. Additional data are taken for the same magnet orientation and range of field values but at several different frequencies on the rf oscillator. The features on the new traces are labeled in the same manner as the original trace and the field values of corcesponding features are then plotted on a graph of magnetic field versus frequency to the negative one-third power. The ralue of the exponent for the frequency follows frow the assumption that the skin depth in Equation 13 will be given by the anomalous skin depth. A sample plot is shown in Figure $6 b$ and it is seen that the lines do converge toward a comon field value in the limit of extremely large frequency values where the skin depth rould 

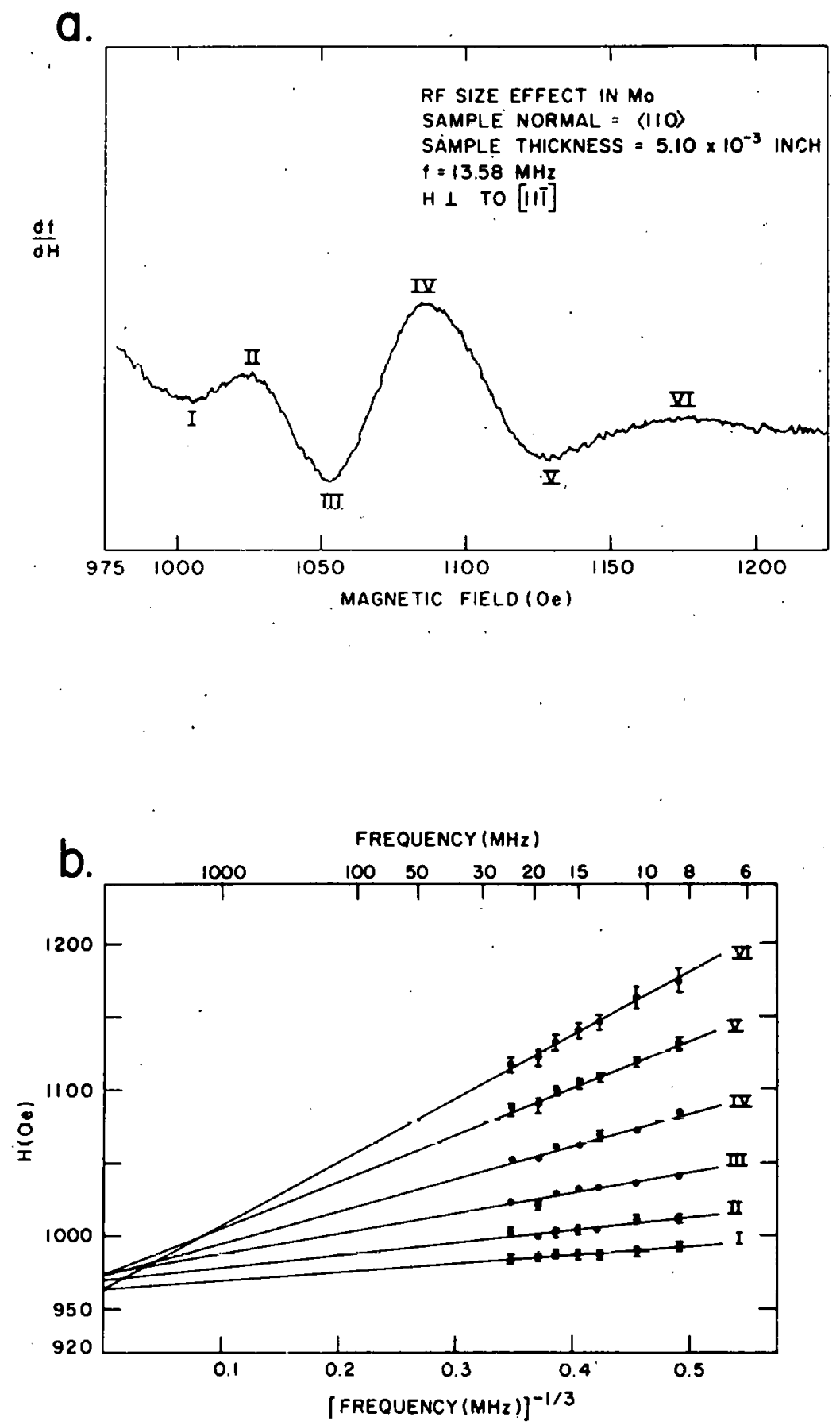

Figure 6. A RFE data trace (a) and the resulting frequency study (b) are shown from cleveland's data on molybdenum (51). Numerals correlate RFSE signal featuras and frequency study lines 
approach zero. This method has been used in several investigations to determine magnetic field values corresponding to the onset of the resonance condition $(52,53,50,54$, $55,56)$

Throughout this last discussion, the detailed behavior of the electric field in the skin depth has been ignored. The ideas discussed in this semi-classical approach to the problem lead to a good picture of the physical processes involved but do not give information relating to the actual form of the anomaly or the actual rf fields found inside the surface of the sample. Theoretical calculations by Raner (57) and Juras (58) examine the forms of the rf fields inside the sample and the resulting forms for the surface impedance anomaIY.

Another aspect of the RSE that has been experimentally observed is the coupling of several electron trajectories in the sample to yield a new surface impedance anomaly at a combined magnetic field value. Consider Pigure 7 for this discussion. Begin with two fS orbits (\#1 and \#2) whose anomalies had been observed at field values of $\mathrm{H}_{1}$ and $\mathrm{H}_{2}$ respectively. As the field value is increased both of these trajectories shrink in real space, but some trajectories represented by 1 will still pass through the skin depth at the surface of the sample. The trajectories now pass parallel to the surface again at a depth in the sample given by 


\section{REAL SPACE TRAJECTORIES}

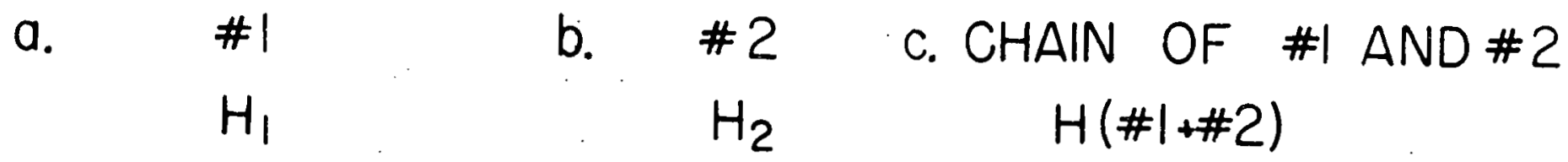

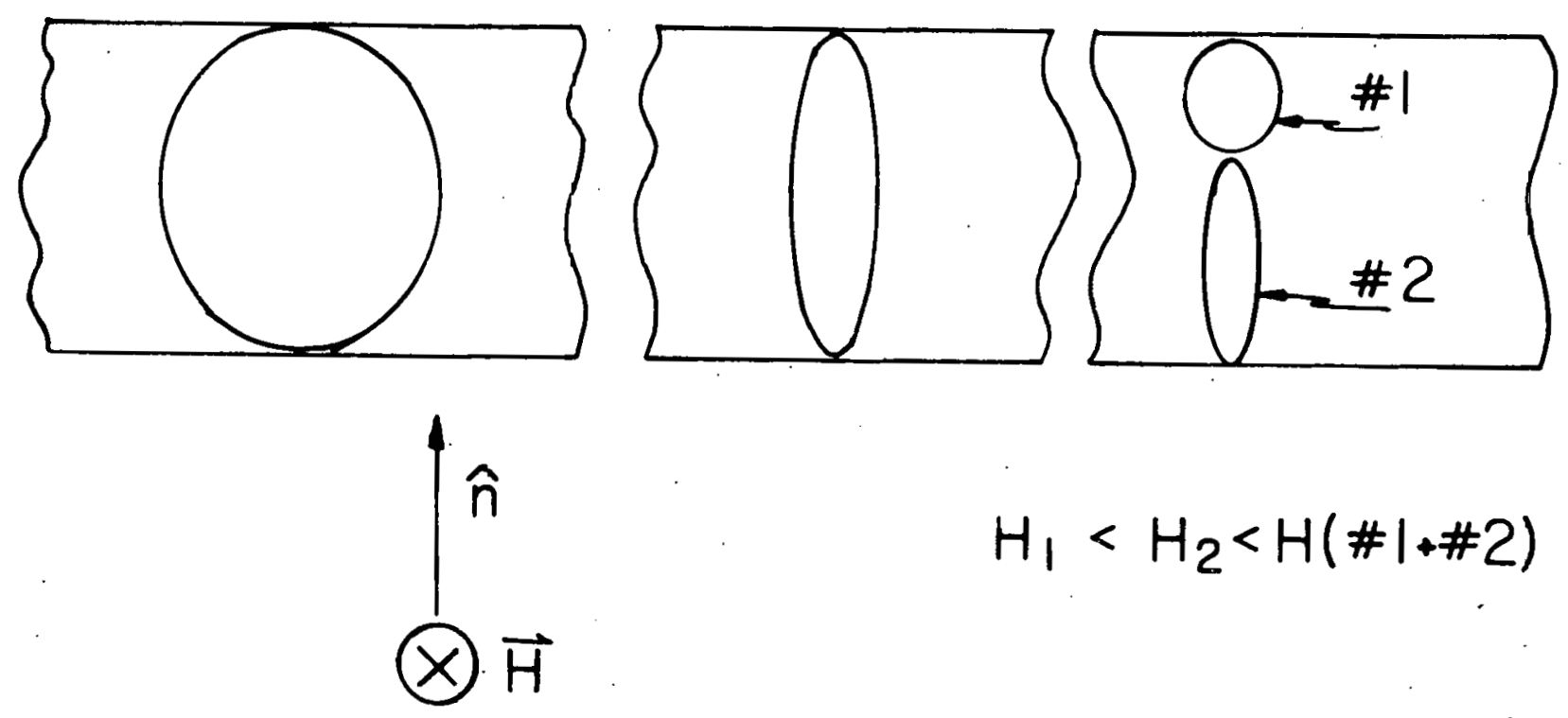

Figure 7. The geometry of coupled orbits is shown. Orbits $\# 1$ and \#2 occur individually of magnetic field values indicated in (a) and (b). The chain occurs at $H(\# 1+\# 2)=H+H$ as seen in (c) 


$$
D_{1}=t\left(H_{1} / H\right)
$$

where $H=c u r r e n t$ value of the magnetic field. This gives rise to a thin layer of current inside the bulk of the sample which is parallel to the sample's surface (roughly as thick as the skin depth) and contains information about the rf fields at the surface. These current splashes have been found and discussed in the theoretical calculation by Juras (58). This internal current splash can now couple to other trajectories to span the remainder of the sample. For such coupled orbits it is easily shown that

$$
\mathrm{H}(\# 1+\# 2)=\mathrm{H}_{1}+\mathrm{H}_{2}
$$

where $H(\# 1+\# 2)$ is the field value for the anonaly due to the coupled trajectories and $\mathrm{H}_{1}$ and $\mathrm{H}_{2}$ are the field values for the single trajectories respectively. If a trajectory couples to an identical trajectory, then joubling, tripling, etc. will occur. Takle (59) observed chains for one orbit containing up to seventeen identical trajectories linked together.

The RFSE was first observed in tin by Gantmakher (49). The evolution of the ideas from cutoff effects in cyclotron resonance experiments to the RFSE is discussed by Takle (59). Early works using the RPSE were primarily aimed at gaining better FS calipers for a wide fange of metals. But as the number of metals left for stud decreased, the RFSE technique was turned toward new information. Several good review 
articles concerning the RPSE technique have been witten $(60$, 61. 62). BFSE studies have been carried out on the following metals:

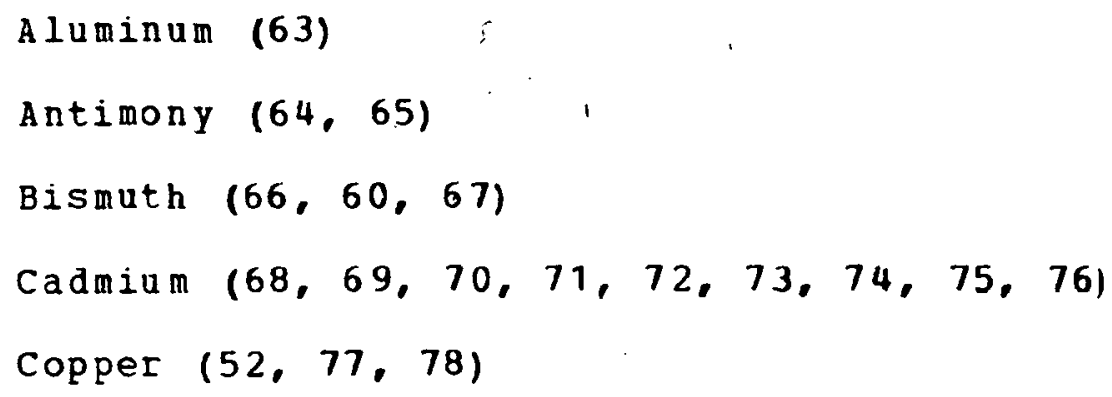


onance 1 ines $(72,116,58,133,134,135,136,87,92,57)$, and orbit chains $(94,60,73,113,118,58,57)$.

Recently the RPSE has been turned toward studies other than FS topology. Among these studies have been determinations of electron relaxation times (114), skin depths $(82,50,87)$, and electron mean. free paths $(120,74$, $88,50,100,104,123,130,126,76,67,93,65,137,138$, 106). Many of these studies were not aimed solely at these parameters but gained the information in addition to the quantities of primary interest to the investigators.

B. The Radio-Frequenay Size Effect: Prequency studies The concepts involved in the frequency study technique for determining the correct value of magnetic field for a given RFSE resonance have been introduced previously. The technique is based on the narrowing of a RFSE resonance as the skin depth decreases with increasing frequency. This behavior was first noted by Rrylor and Gantmakher (50). Since then it has been used by many $172,52,53,50,54,55,98$, $103,113,114,56,119,108,57$ ) in an effort to determine the correct magnetic field values to use in Equation 9.

Several recent RPSE studies have been performed on molybdenum $(95,96,54,55,51,139)$ with differing results. Tho PS calipers obtained in these studies show the same angular dependence except they differ in magnitude by about five per cent. A recent DHVA study of molybdenum (140) has 
found good agreement with Boiko et al. $(95,55,139)$. Boiko et al. In their early rock chose the magnetic field value corresponding to the loy field end of the resonance feature. Cleveland (51) and Cleveland and Stanford (96, 54) aade extensive use of the frequency study technique to determine field values for the different resonances observed. The use of the frequency studies apparently led to values of the magnetic field that are five per cent too small. Figure 8 shous a Cleveland data trace (51, Figure 35) and inzludes tro arrows marking the values of magnetic field for the resonances as found in the frequency study technique (Hres) and also arrows indicating the first departure of the RFSE signal from the background signal (H'): Notize that these resonance field values fall at lover field values than the first departure of the RFSE signals from the background signal. Based on the results of these molybdenum studies it. would be useful to re-examine the way in which the frequency studies were carried out by cleveland.

RFSE data were taken with all experimental conditions the same except the frequency of the rf oscillator. From these data traces, individual features were labeled as in Figure $6 \mathrm{a}$. Then the field value at each feature and the uncertainty in that field value were determined by measuring the trace relative to the known end points on the field sweep. The values of magnetic field (H), uncertainty in 


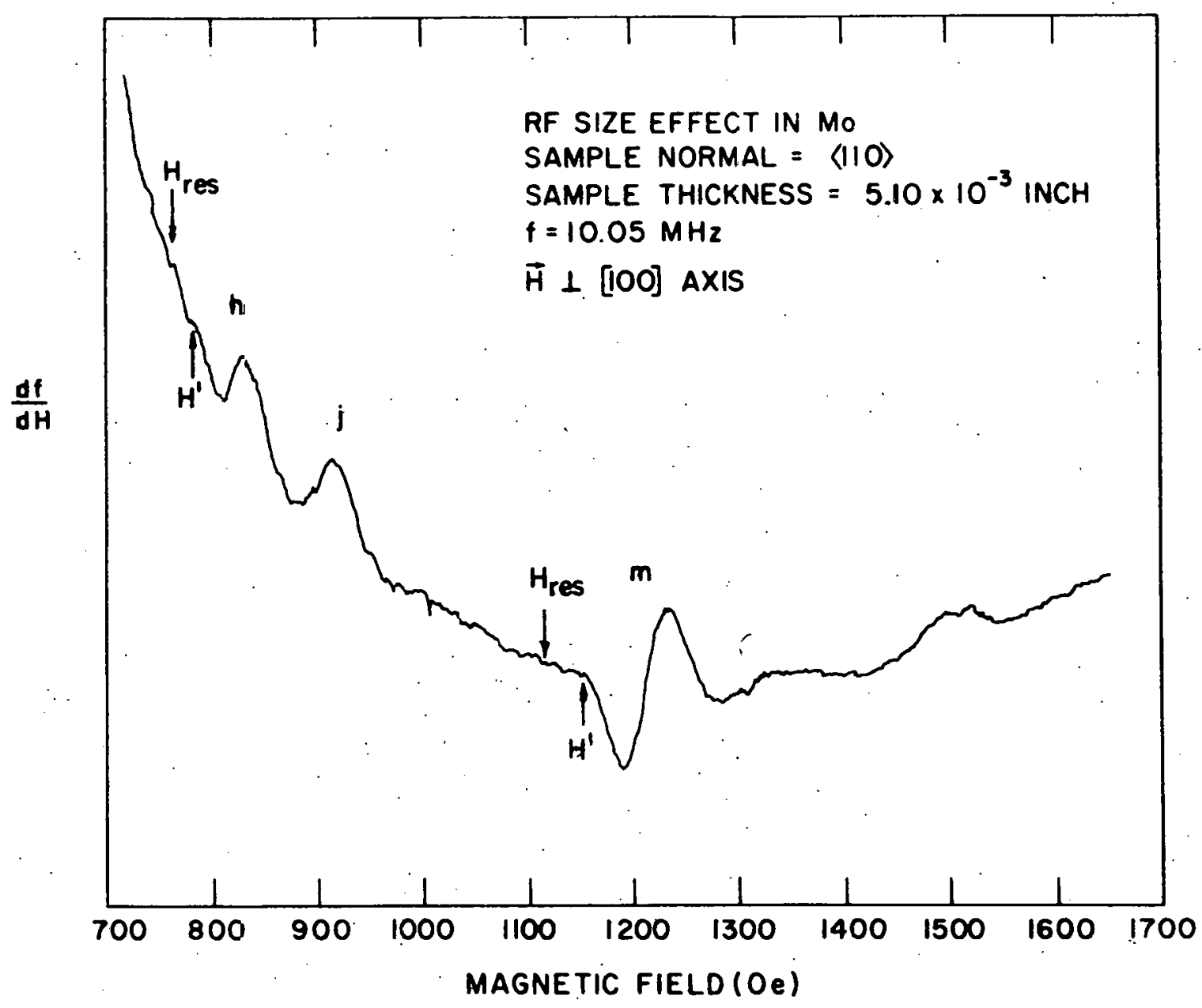

$\underset{\omega}{\omega}$

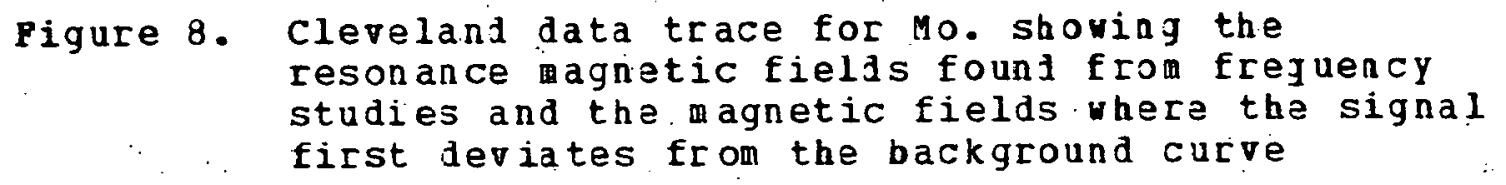


field $(\Delta H)$, and freguency (f) were then known for each feature measured. The information for the same feature at all different frequencies was grouped together as these points will contribute to a single line on the frequency study as seen in Fiqure $6 \mathrm{~b}$.

The data for each line were then used to carry out a linear least-squares fit to a line given by

$$
H=A+B f-\infty
$$

where $A=i n t e r c e p t$ of the line at $f=$ infinity, $B=s$ lope of the line, and $(-\alpha)=t$ he exponent used for the frequency. For this linear least-square fit each point was given $a$ weight inversely proportional to the square of the uncertainty in the field value for the point $(141)$. This process led to values of $A$ and the uncertainty in $A$ for each line at a given value of $(-\alpha)$. For all lines in a group (such as figure 6b) the values of the intercepts were statistically combinad to yield an average intercept $A(a v e)$ and the uncertainty in that intercept $\sigma_{A}$.

This procedure was repated as $\alpha$ was incremented over a range of values that included the frequency exponent for the classical skin depth $(1 / 2)$ and the anomalous skin depth $(1 / 3)$ for a free electron gas. The results were plotted on graphs of $\sigma_{A}$ versus $\alpha$. The value of $\sigma_{A}$ was taken to be the size of the error bar for the value of the average intercept with a $90 \%$ confidence level. For the frequency study on the six 
lines of Figure 9a. (Cleveland Figure 30) the resulting plot of $\sigma_{A}$ versus $\alpha$, Figure $(9 \mathrm{~b})$, shoved a minimum at $\alpha=0.33$. This was taken as confirmation of the agreement with the anomalous skin depth frequency dependence. The values of A(ave) for the remaining frequency studies were then determined for $\alpha=0.33$.

several points should be mentioned concerning these linear least-squares fit calculations.

1) The values of chi-squared (141) for the individual lines are very insensitive to changes in $\alpha$ for the data taken. The chi-squared values were often smaller than unity for large ranges of $\alpha$ and therefore were not a meaningful indicator statistically for choosing a preferred value of $\alpha$. This results from a relatively small range of frequency data, a long distance of extrapolation, and relatively large error bars on the values of magnetic field. Based simply on the value of chi-squared it would be impossible to choose a preferred value of $\alpha$ to give a "best" fit. for a given line.

2) The values of a corresponding to minima in the $\sigma_{\Delta}$ versus $\alpha$ curves do not all have the same magnitude. They are spread over the range from 0.25 to 0.44 . The wide range of values is not encouraging and some of these values (from 0.33 to 0.25 ) fall outside the 

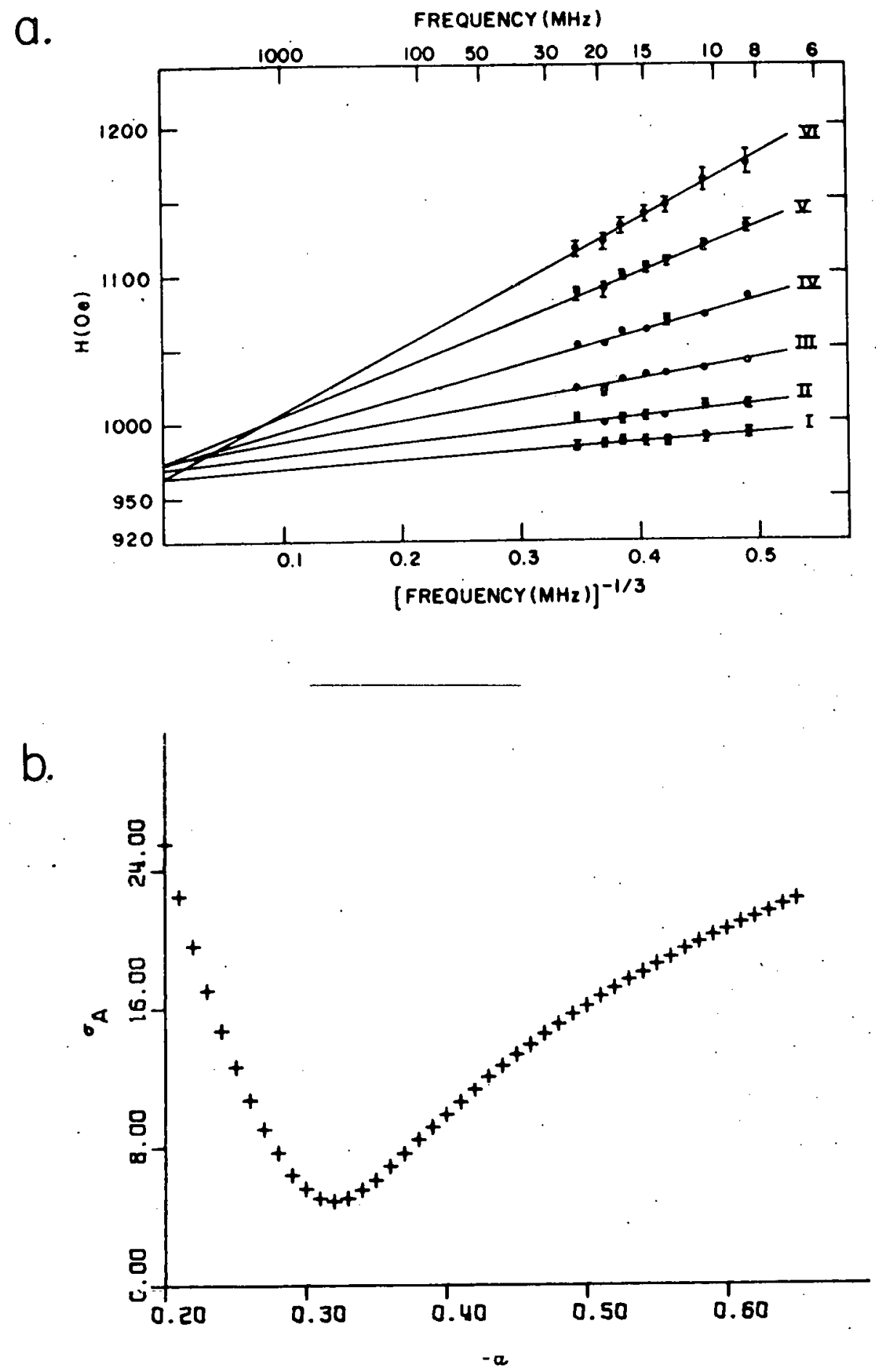

Figure 9. Prequency study (a) and the resulting confidence limits for the mean value of the intercept as a function of the exponent for the frequency dependence of the RESE line broadening (b). Original data after cleveland 
range determined by the classical and anomalous skin depths. The groups of lines examined with three or more lines per group exhibited a smaller range of $\alpha$ from 0.32 to 0.35 .

3) Even if all values of A (ave) were chosen for $\propto 0.33$ the field corrections would not be large enough to account for the five per cent difference with the other molybdenum studies. In most cases the change nould be less than one per cent.

From this brief examination it is evident that the concept of the frequency study and cleveland's method for determining the proper frequency dependence should be examined more closely. One experimental parameter that has not been examined in relation to the frequency study is the uniformity of the sample's thickness.

Consider a wedge-shaped sample whose thickness varies. between $t$ and $t+\Delta$. Examine motion for an electron making a circular trajectory in the sample which is subjected to an If. field at the surfaces. A cross sectional view of the sample and the possible orbits is shown in Figure 10. As was seen in the earlier discussion of RFSE signal broadening due to the skin depth, it is again possible for a variety of trajectory diameters to expose the electron to the rf fields in the skin depth at both surfaces without striking either surface. The two orbits illustrated represent the maximum 


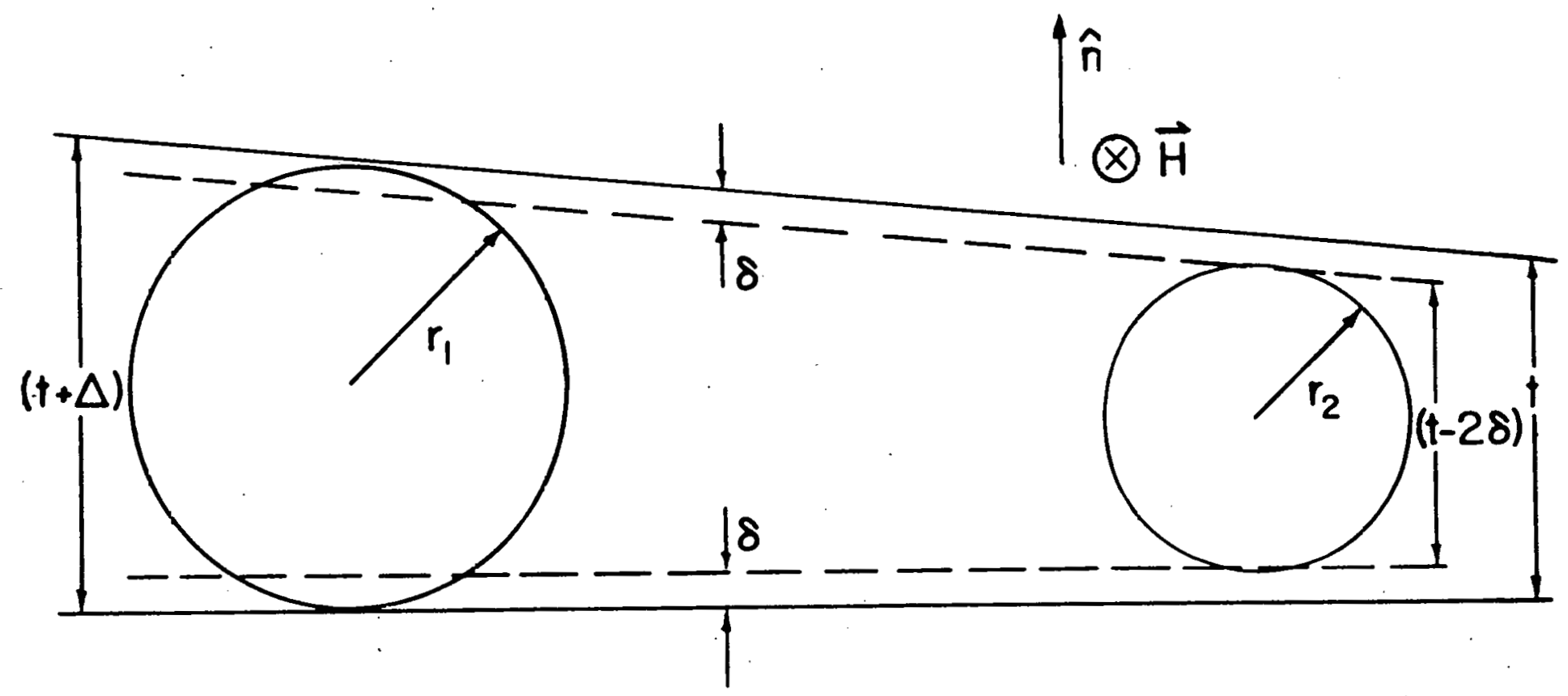

Figure 10. The effects of variations in sample thickness anc the skin depth on the variety of electron trajectories that can contribute to the RFSE are illustrated 
and minimum diameter orbits that can do this and they satisfy the conditions

$$
2 r_{1}=(t+\Delta) \text { and } 2 r_{2}=(t-2 \delta)
$$

Pollowing steps analogous to those used in the earlier discussion of line broadening, this leads to the conclusion that this electron orbit can contribute to a BFSE resonance over a range of magnetic fields. The fractional width of the RPSE resonance can be written as

$$
\Delta H / H_{1} \cong(\Delta+2 \delta) / t \text {. }
$$

The effect of this broadened contribution to the RFSE resonance must be examined to determine the effect expected on the signals observed experimentally.

For the electron trajectory considered, the electron will be able to interact with the rf fields and contribute to surface currents and surface impedance equally well for all values of magnetic field between $H_{1}$ and $H_{2}$. The resulting effect on the surface impedance is schematically represented in Figure 11a. But the RFSE signals are proportional to the field derivative of the surface impedance and may be expected to show some similarity to the curve shown in Figure $11 \mathrm{~b}$. Notice the splitting into two signals and the inversion of the two signals on the derivative plot relative to each other. This is exactly the kind of behavior mentioned by Gantmakher (60, Figure 19) for a wedge-shaped sample. This simplified, classical argument has again neglected the actual 
a.

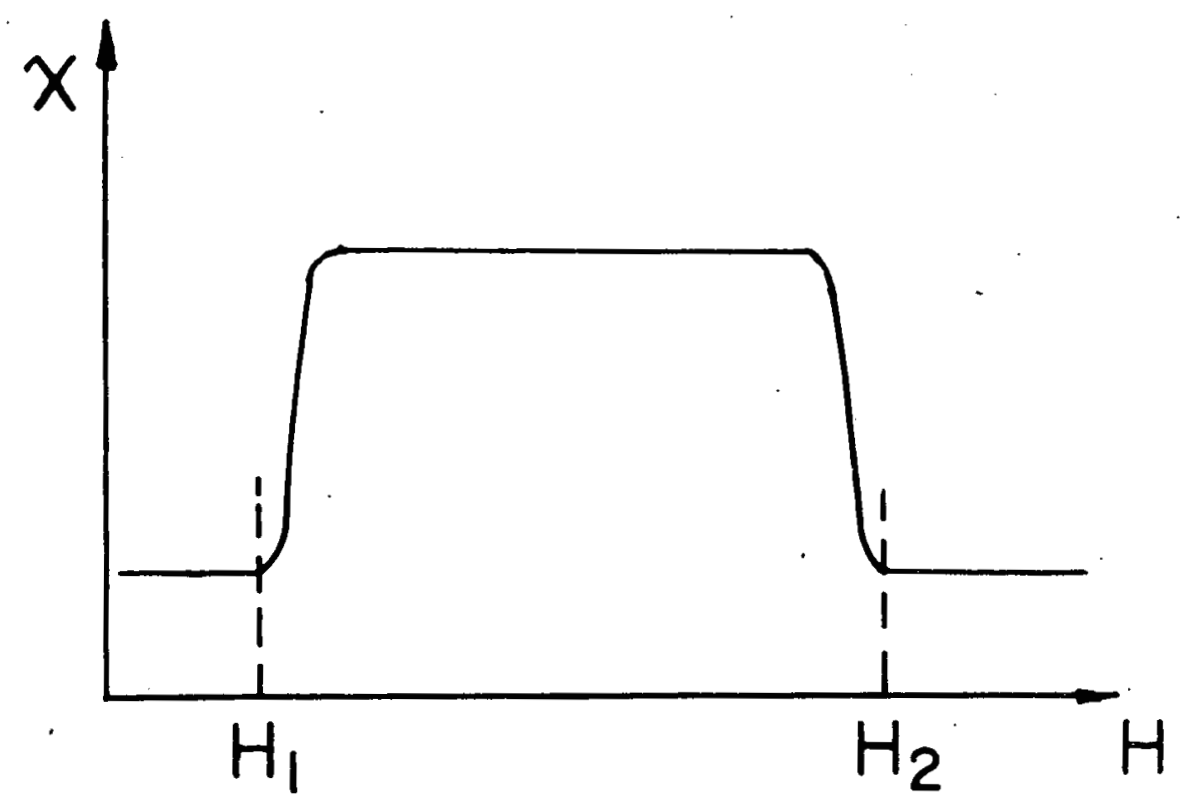

b.

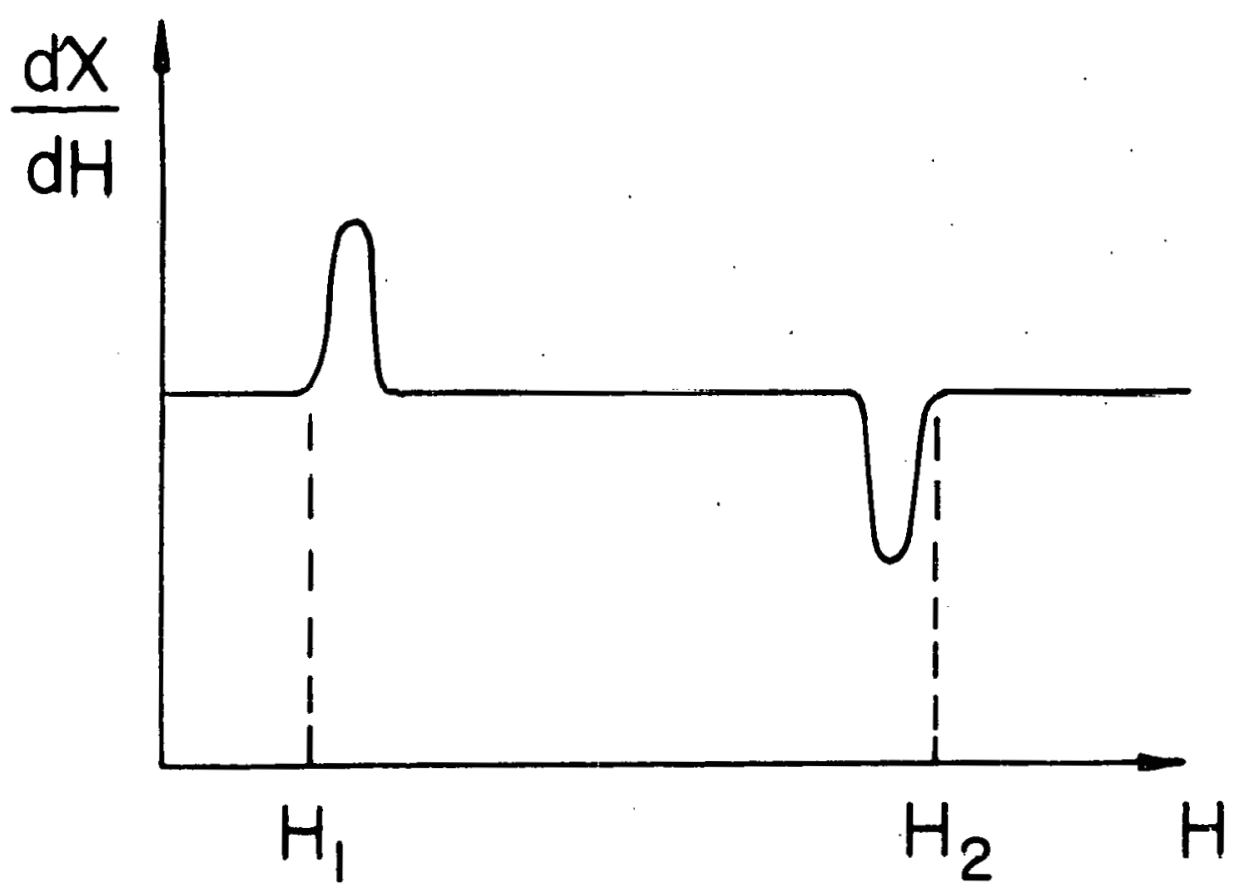

Figure 11. A schematic representation of the effect of a redge-shaped sample on the surface impedance is shorn (a). The resulting RFSE signal detected in this investigation is shown (b) 
form of the rf fields in the sample and should be used only to gain insight into the type of beharior expected. Exact agreement ith experimental line shape should not be expected.

Several authors $(60,74,50,119,108,135)$ have noted that a non-uniform sample thickness can lead to signal broadening rather than signal splitting as seen above. Consider the case where the sample surfaces are undulated rather than simply flat but non-parallel. Then for different values of aganetic field between $H_{1}$ and $H_{2}$ different numbers of electron trajectories may contribute to the surface currents and surface impedance. one possible form of the resulting surface impedance is shown in pigure 12 a and the resulting field derivative is shoun in Figure 12b. The exact forms of these curves would depend on the actual pattern of thickness variations in the sample.

These two cases have indicated that non-uniforn sample thickness will generally lead to broadening of the RFSE signal and distortion of the line shape from the form that would be observed in a perfectly flat sample. For the special case of a perfectly wed ge-shaped sample, RPSE signal splitting will occur.

From Equation 18 it is seen that the non-uniforaity in sample thickness and the skin depth affect the RFSE resonance width in the same functional manner. The relative magnitude 
a.

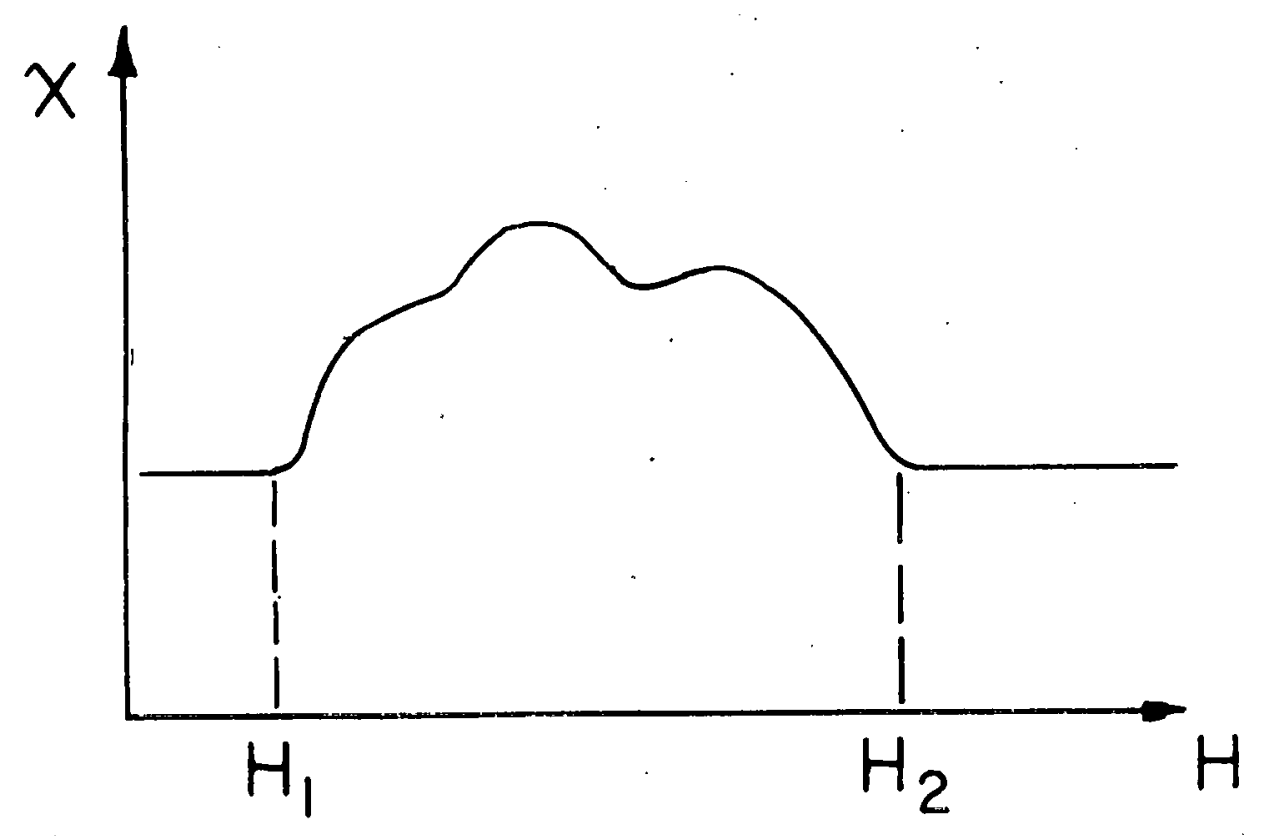

b.

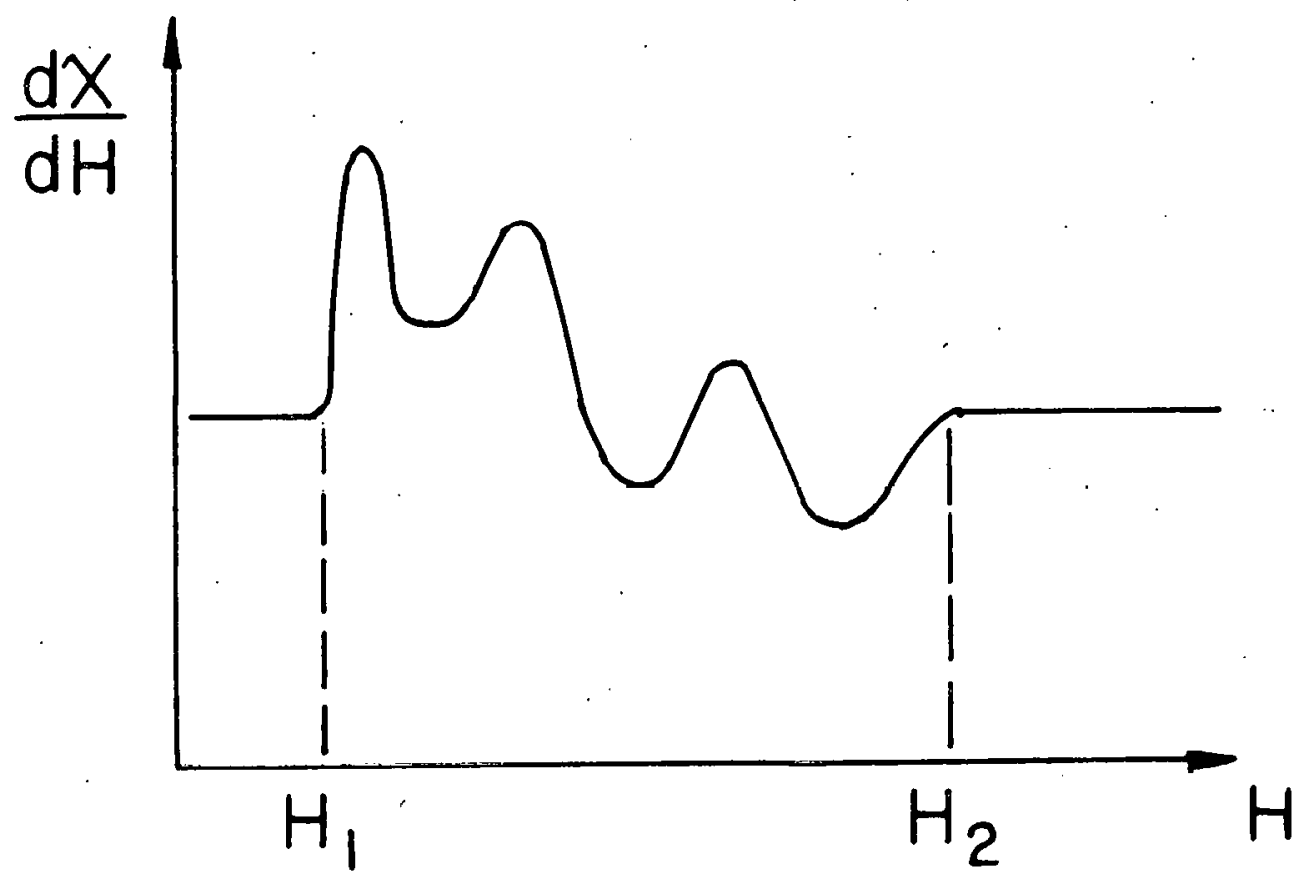

Figure 12. a) A schematic representation of the effect of random variations in the sample thickness on the surface impedance is shown. b) The resulting RFSE signal datected in this investigation is shoun 
of the two factors can be evaluated by examining a frequency study plot and Equation 18. For infinite frequency, the RFSE line width is governed by the non-uniformity of the sample thickness.

$$
\left((\Delta H) / H_{1}\right)_{\infty}=\Delta / t
$$

Then at a finite frequency, the line width is the sum of the frequency independent term given in Equation 19 and the standard broadening due to the skin depth as first seen in Equation 13. If the skin depth is taken to be

$$
\delta=\operatorname{af}^{-\infty} \text {. }
$$

then the skin depth broadening is directly proportional to the coefficient "a" in Equation 20. Thus the slope of the steepest line on a frequency study is directly proportional to the coefficient seen in this simplified exprassion for the skin depth.

The line width at any frequency can be broken into the width due to the skin depth and the width due to thickness variations. The line vidth due to the skin depth is proportional to the difference in the slope of the steepest and flattest lines in a given frequency study. For example:in Figure 9a, the slopes would be measured for lines I and VI. The difference in these slopes is then an indication of the importance of the skin depth in broadening the RPSE sigaal. The line width due to saple thickness rariations is found by examining the frequency study lines for infinite frequency. 
The spread in their intercepts is an indication of the importance of thickness variations in broadening the RFSE signal. The division of the largest difference in the intercepts by the largest difference in slopes results in a number that indicates the relative importance of the thickness broadening and skin depth broadening. The quantity resulting from this division has been called RATIo and will be used on several figures. As Rar Io decreases the thickness broadening becomes less important relative to the broadening due to the skin depth. RATIo is equal to zero for a perfectly flat saple and the skin depth is the only source of line broadening. As Ratio increases, the sanple quality is decreasing as the sample behaves less and less like the thin, flat sample wanted idea $11 \mathrm{y}$.

The effect of this broadening on cleveland's method for determining the proper frequency dependence for the line width and the correct field ralue has been examined. This was done by generating data points that fell on lines showing the frequency dependence seen in Equation 16. Systematic variations were included in the intercepts of the indiridual lines and in their slopes. These variations thus incorporated changes in the flatness of the sample and the magnitude ofthe rf skin depth. Data ere generated for five frequencies between $2.4 \Delta \mathrm{Hz}$ and $18.6 \mathrm{MHz}$. 
The generated data sets were examined using the Cleveland linear least-square fit method for the individual lines. The value of the frequency exponent, $\alpha(m i n)$, was found that gave the least uncertainty. $\sigma_{A}$, in the common intercept, $A(a v e)$. This corresponds to the $\alpha$ at the minimum on a $\sigma_{A}$ versus $\alpha$ plot as seen in Figure 9b. Figure 13 illustrates the dependence of $\alpha(m i n) / \alpha($ real) on Ratio, the relative importance of the thickness broadening compared to the skin depth broadening. Data are shown for $\alpha($ real 1$)=1 / 3$ and 1/2. Both data sets show a very similar dependence on RATIo with only small differences in the value of $\alpha($ min) $/ \alpha($ real) for a given value of Ratro. From this it is seen that for perfectly flat samples, RATIo=0, Cleveland's technique yields the proper value of the exponent seen in Equation 16. The value of $\alpha$ indicated by the cleveland method decreases as the sample becomes less flat. Any variztion in sample thickness yil cause the resulting $\propto$ to be lower than the actual value.

This calculation showed that $\alpha(m i n) / \alpha($ real) depended only on the relative importance of the two sources of broadening and not their absolute magnitude.

The effect of a non-uniform sample thickness on the common intercept resulting trom the cleveland technique, A (ave), has also been examined. The common intercept is examined relative to the lowest intercept of the frequency 


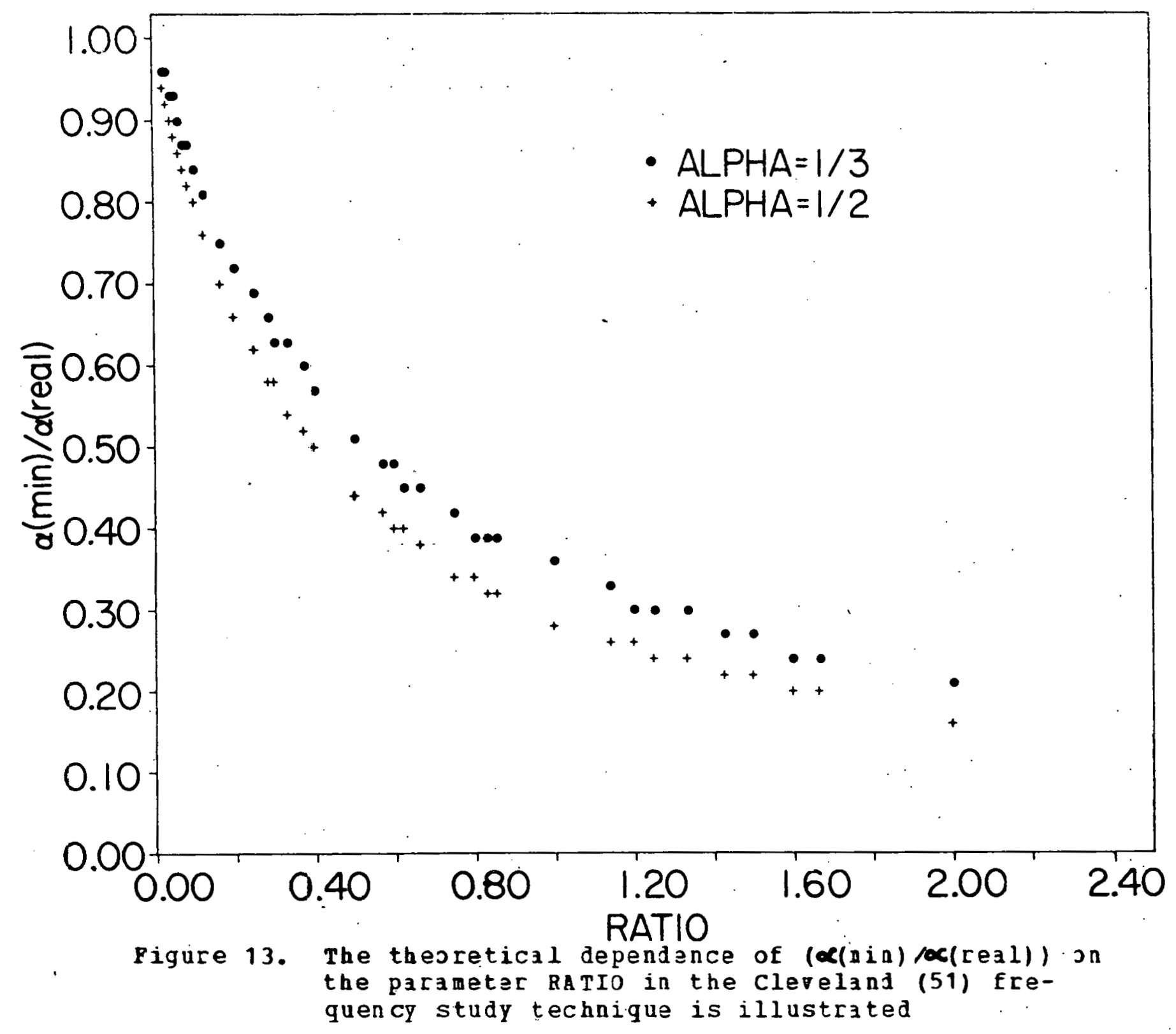


study lines when they are examined using Equation 16 with the frequency exponent taken-as $\alpha(r e a l)$. This lowest real intercept will be denoted A(min). Pigure 14 illustrates the dependence of (A (min)-A(ave)) versus the paraneter BaTro. Positive ralues on this vertical scale represent amounts that A (ave) is reduced belor a(min). Fron the figure it is seen that the reduction depends only on spread in the intercepts of the lines in the frequency study. It can also be noted from figure 14 that the value of (A(min)-A(ave)) is roughly the same size as the spread between the intersections for the lines in the frequency study. The points are grouped about horizontal lines so they show no functional dependence on RATIO. Hence the reductions do not depend on the skin depth or its frequency dependence.

This behavior can easily be understood by considering the form of the data. Pirst consider the data plotted on a graph of magnetic field versus $f^{-\alpha}$ where the actual exponent for the data is used. Assume the case where two frequency study lines exist and they do not have a comon intersection. If the value of $\propto$ for the plotted lines is increased without changing the horizontal scale, the lines will bend upward in the region near the vertical axis. The upper line will bend up more than the lower line because its slope will be larger than that for the lower line. Thus the intersections vill diverge as the axponent is increased. This is a direct 


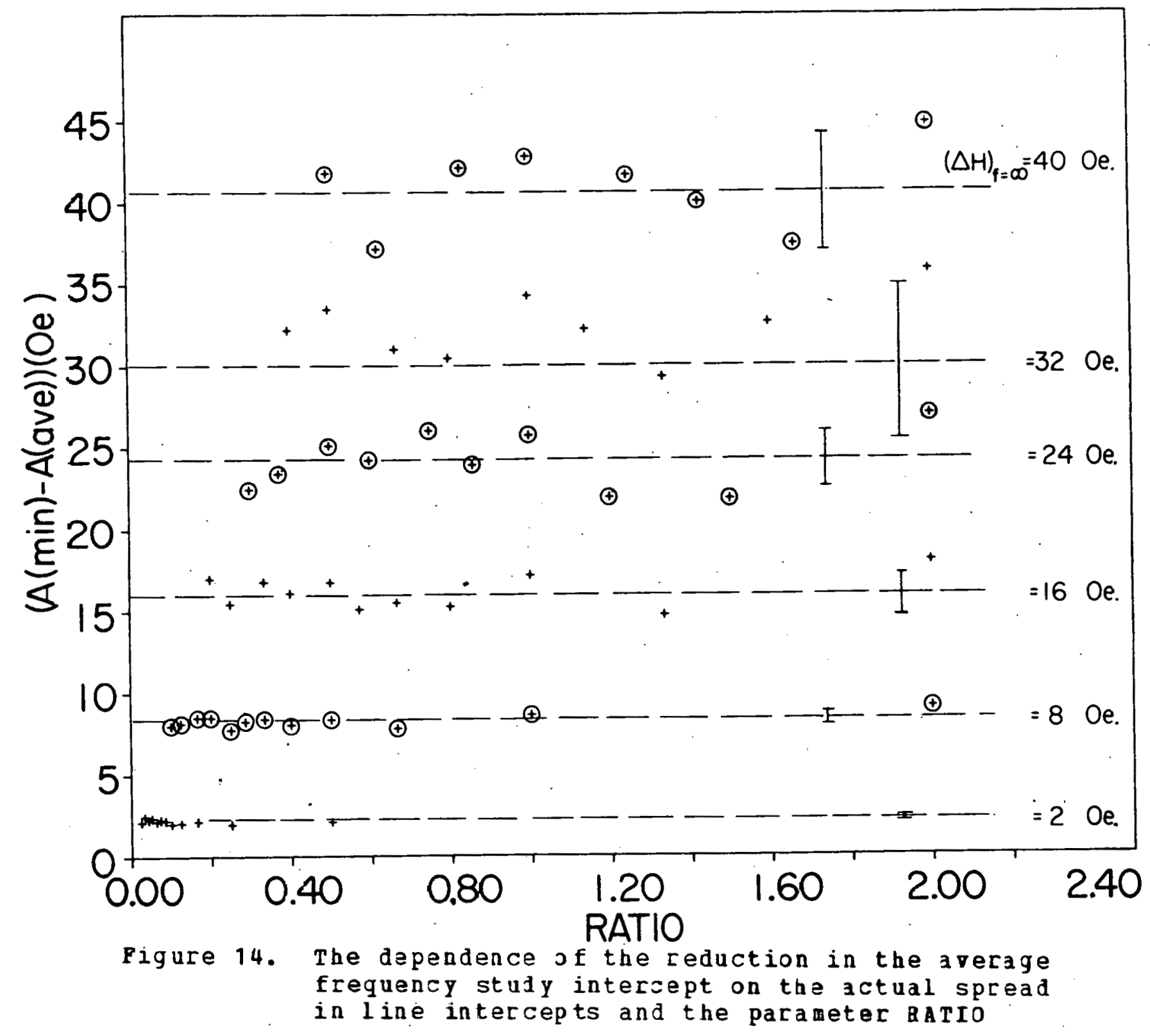


result of the fact that for the data being considered, $f-\alpha$ is always less than unity.

Next consider decreasing the value of $\alpha$ for the plotted 1ines. Again leave the horizontal axis unchanged. Now the two curves bend downuard in the region near the vertical axis. The upper line is again bent more because of its larger slope. Thus the intersections for the two curres converge and for some $\alpha($ min) <a(real) the spread between the intersections will be minimized. This corresponds to the value of $\alpha(m i n)$ selected by the cleveland techinique. It is seen that any spread between the intersections will cause the indicated $\alpha(m i n)$ to be less than $\alpha($ real). It is also seen that the common intercept at $\alpha($ min) will be smaller than the actual intercept found for $\alpha($ reail).

The effect of a non-uniform thickness has been examined on both the exponent and the intercept resulting from cleveland's frequency study method. Deviations in sample thickness have been found to cause the resulting values of $\alpha(m i n)$ and a (ave) to be lower than the correct values. Throughout this discussion the If field has been assumed to have only a single frequency component. The affects of possible frequency harmonics present in the rf fields have been ignored.

The presence of harmonics in the rf field would further complicate the frequency study technique. Suppose the rf 
fields at a sample's surface have components at $f_{0}$ and $3 f_{0}$. By examining a frequency study plot it is seea that the $3 f_{0}$ RFSE resonance will occur at a lower magnetic field value than the fo resonance. The two resonances would combine in some manner. The resulting resonance could be expected to begin at the lon field end of the $3 f_{0}$ resonance and extend to the high field end of the $f_{0}$ resonance as seen in Pigure 15, but the frequency counter would indicate $f_{0}$. The result of this would be a frequency study plot where the data points would be located at magnetic field values that vould be artificially low. The confusion could be compounded if the harmonic content of the rf signal depended on the oscillator frequency as seen for tube oscillator $A$. In this case the amount of shift toward lover field values vould be proportional to the amount of harmonic content in the rf signal. From the preceding discussions, several conclusions can be reached about the frequency study technique for determining the magnetic field were the RSE resonance begins.

1) Efforts must be made to ensure that the rf signal applied to the sample is a pure sine wave with virtually no harmonic content.

2) Variations in the sample's thickness can leat to signal broadening and a finite RPSE line width for 1afinite frequency. To aroid this, efforts must be directed at minimizing the deviation of the sample from 
o.

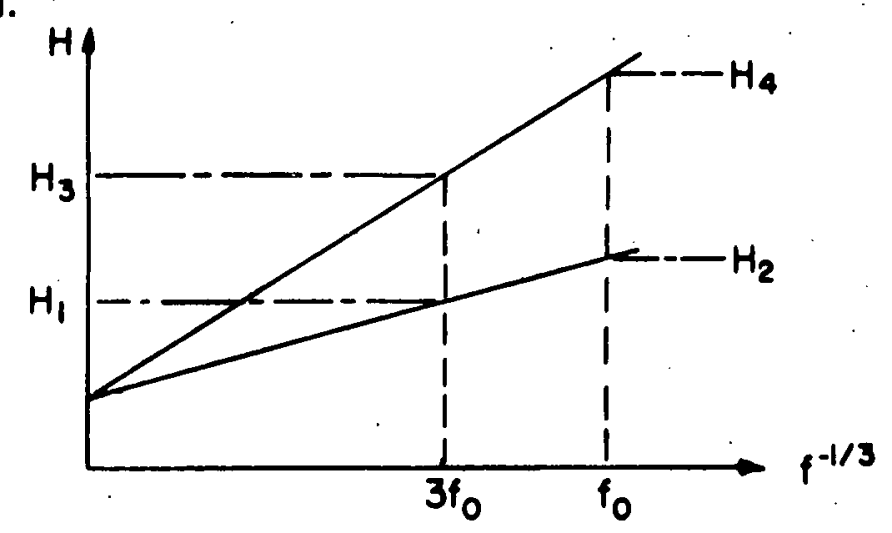

b.

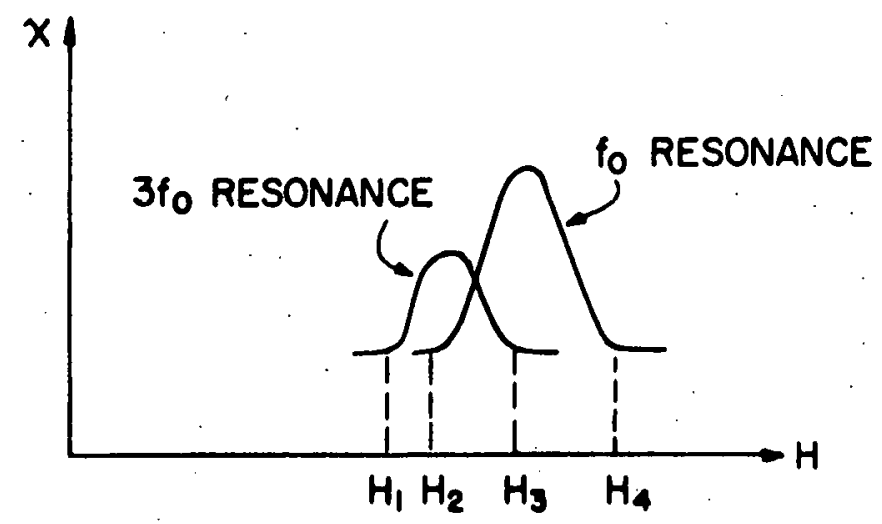

c.

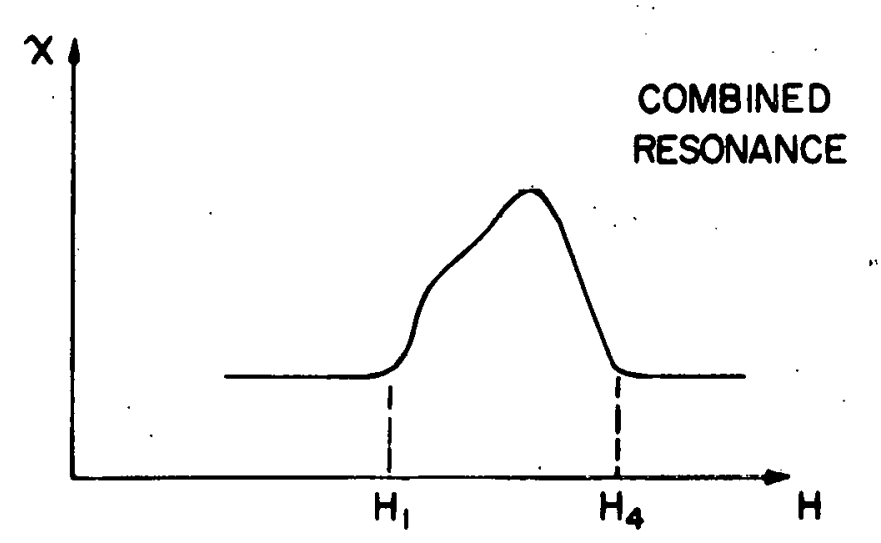

Figure 15. The effect on the surface impedanze is illastrated for a ReSE resonance exposed to a signal containing frequencies $f_{0}$ and $3 f_{0}$ 
a flat plate. Osing small area samples ay be helpful for this. If the two surfaces of a sample are slight-. 1y misaligned, the variation in thickness is then proportional to the length of the flat surfaces. A $0.5^{\circ}$ misalignuent between the two surfaces can result in $a$ difference of 70 aicrons in the thickness at opposite ends of an 8 ma sample. Therefore smaller sample area leads to smaller variations in the sample thickness.

3) Efforts nust be directed torard minimizing the uncertainty in the field values of individual line features. This yould allow a better determination of the frequency dependence of the individual lines in $a$ frequency study group. Snaller ercor bars on the magnetic field would ake the chi-squared values more aseful for the individual lines. This could be done by taking duplicate data traces and determining the average magnetic field for a feature and its standard deriation.

4) Extending the frequency range stadied would a lso help make the chi-square values more useful for determining the frequency dependence of the individual lines. 
IV. RADIO-PREQUENCY SIZE EFFBCT SIGNALS IN ALUMINOM: COMPOTER PREDICTIONS

A serious problea can exist in RFSE experiments when one attempts to associate individual signals with the appropriate Fermi surface calipers. Roach (94) eaphasized this in his discussion of the RPSE technique and the PS of magnesium. Many signals were seen and orbit assignments would have been very difficult without an accurate FS model. This problem is compounded by the fact that orbits can form chains to give signals at magnetic field values that do not correspond to $z$ single FS caliper. This effect can give rise to doubling for tripling, etc.) if the orbits are the same or to totally new shapes on the plot of caliper versus magnet angle if tro different orbits couple to yield one signal. Roach even noted an orbit that did not give $r$ ise to a signal by itself but did appear coupled to another orbit that had been identified.

To avoid some of these problems in this experiment, Harrison's model (13) for the second zone hole surface of the FS of aluminum ras used to calculate the expected calipers for different sample normals and magnetic field angles. The experimental data for a given sample normal yield signals of varying strengths that can be analyzed to obtain plots of caliper versus majnet angle for the given sample. Thus it was desirable for the calculation to yield plots of caliper versus magnet angle plus information about the anticipated 
signal strengths at the different angles considered.

To carry out this calculation it is necessary to analyze Harrison's second zone model (Figure 16). The surface is completely determined by fourteen spheres of equal radii and their intersections. So the problem. is reduced to a relatively straightf or wari geometrical exercise. The calculation can be broken into four major areas.

1) Initially the coordinate system $(X, Y, z)$ is chosen so the axes are parallel to cubic axes of the crystal. The coordinate system is then rotated using Euler's angles (142, p. 458) such that the new $z$ axis (z') is parallel to the desired sample normal. This rotation simplified many of the algebraic equations used later in the program to calculate sphere intersections and to allow determination of actual caliper dimensions. In the most complicated part of the program, the rotation reduced many calculations from three dimensions to two dimensions. The new coordinate axes will be labeled $x^{\prime}, y^{\prime}, z^{\prime}$

2) Then fifty-seven (57) equally spaced planes perpendicular to the $z^{\prime}$ axis (the sample normal) are passed through the Bz with the central plane always passing through the center of the B\%. The spacing hetyeen planes was selected to be large enough that for any possible sample normal the second zone fS would not 


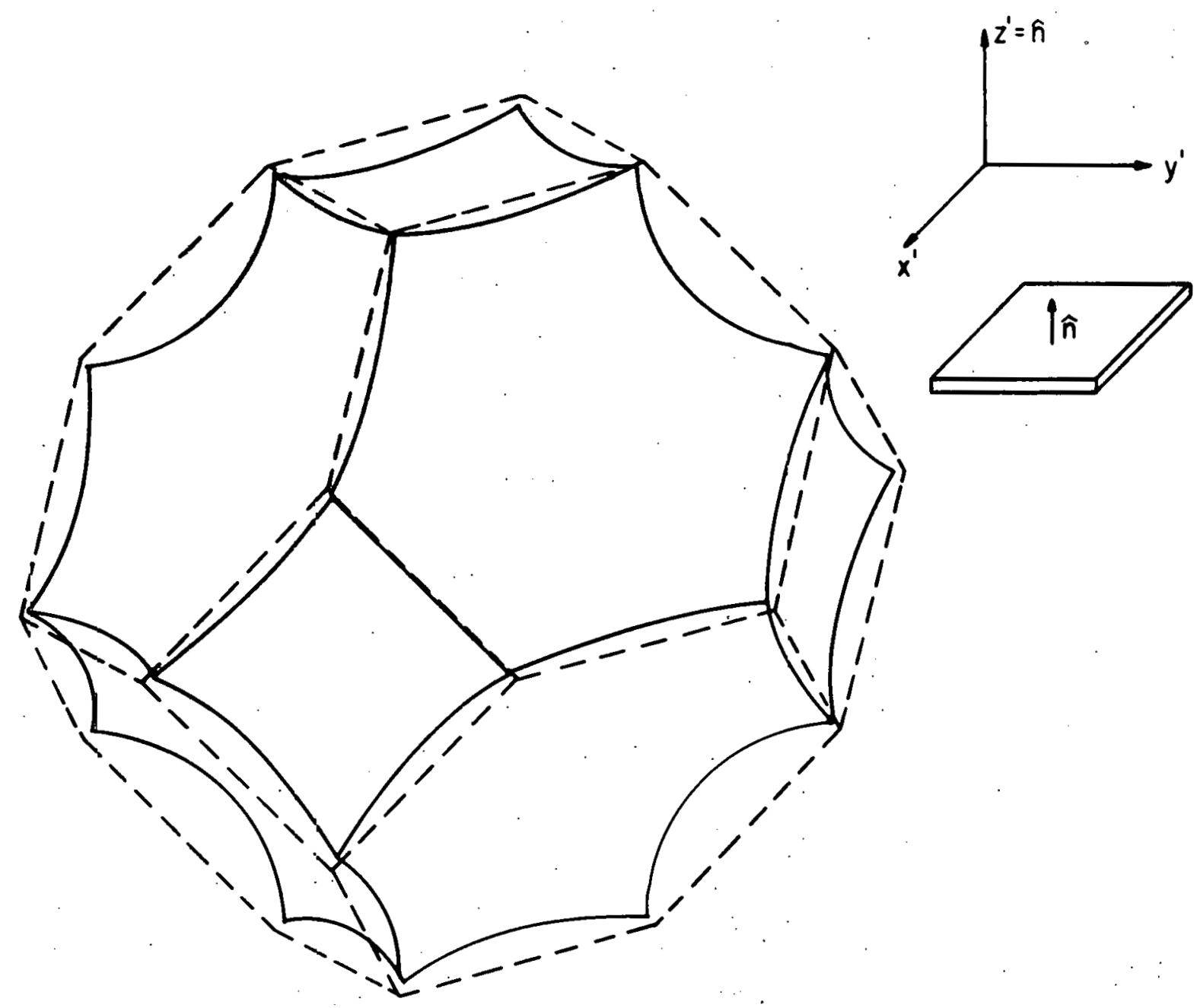

Pigare 16. The aluminum NFE second. zone PS shown with the sample geometry. The coordinate system indicates the coordinaties used in the computer calculation following the initial coordinate rotation 
extend beyond either of the outside planes. Each plane is then examined for intersections with the sezond zone PS.

Clarification of several terms should make the remaining discussion easier to understand. As seen earlier, when a constant magnetic field is applied to a material, the electrons move in k-space on constant energy surfaces in planes perpendicular to the magnetic field. The path in $k$-space followed by such an electron around the FS (a constant energy surface) uill be called an orbit. In the present geometry with the magnetic field applied perpendicular to the sample normal ( $z^{\prime}$ axis), this means that electron orbits will be in planes parallel to the $z^{\prime}$ axes and perpendicular. to the magnetic field.

Now consider the intersections just found for the second zone PS and the 57 planes of constant $z^{\prime}$. These intersections are in planes parallel to the magnetic field direction so they do not contain actual paths traversed by the electrons. These intersections simply give the cross section of the fS for a specific value of $z^{\prime}$ and will be referred to as cross sections. Figure 17 shows three sample $z$ ' planes and their resulting cross sections with the second zone FS. 


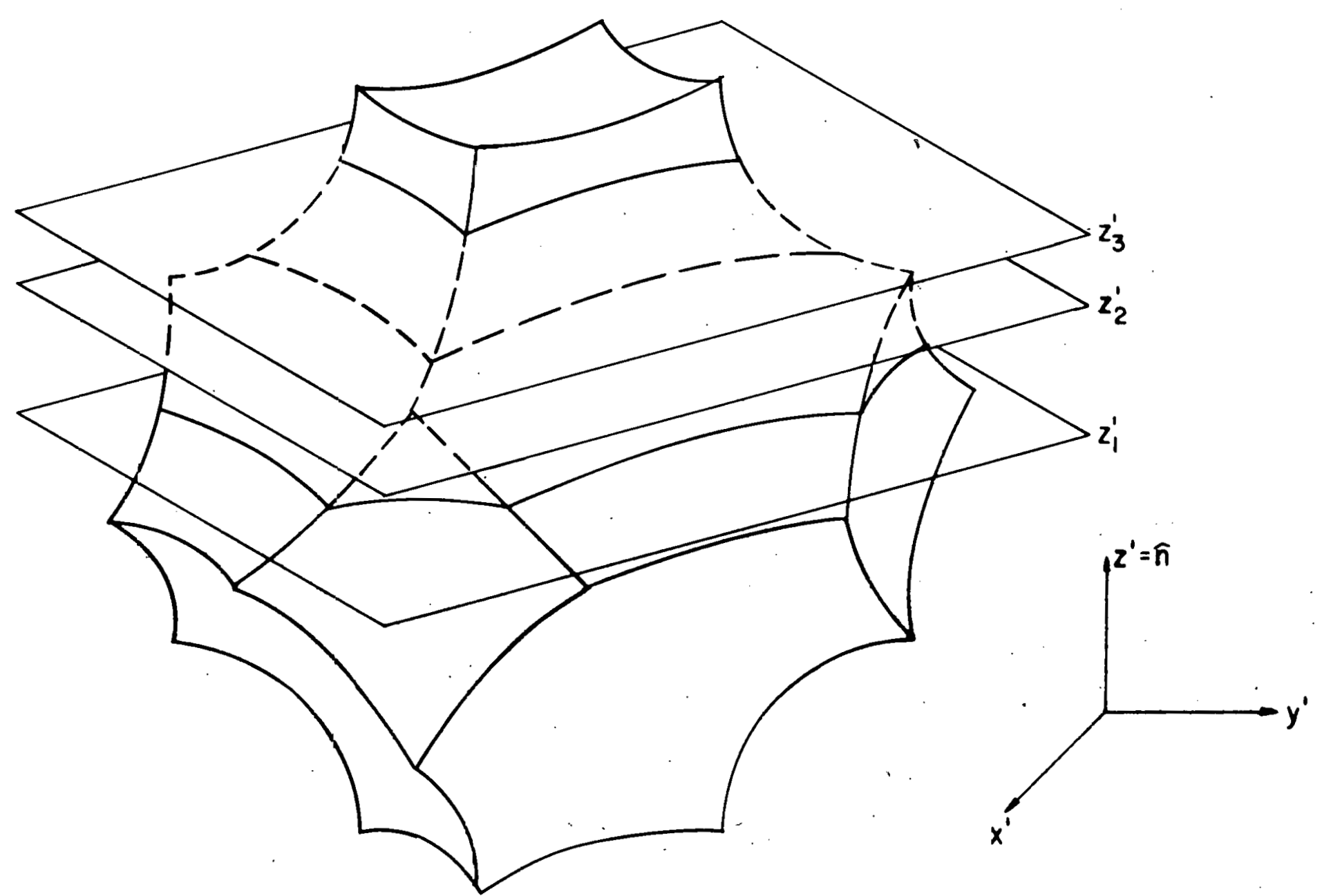

Figure 17. The aluminu NPE second zone FS shown with

three typical planes perpendicular to the sample normal as used in step 2 of the computer calculation. The intersections of these planes and the PS result in cross section outlines 
Following the examination of all 57 slices, the information pertaining to each cross section was stored for use later in the program. The number ofcross sections found on a single slice varies from zero to six. The information can also be used to plot the cross sections found on each.z' slice. For examples see Figure 18, which shows cross section plots for a [100] normal sample for $z:=0.00$, $z^{\prime}= \pm 1.3750 \AA^{-1}$, and $z^{\prime}= \pm 1.4375 \AA^{-1}$, respectively.

For $z^{\prime}=0.00$ the figure is the central cross section of second zone FS. The four curves that the cubic axes pass through are diagonals of the foursided faces. Each of the remaining four sides represent the common side between two hexagonal faces. For $z^{\prime}= \pm 1.3750 A^{-1}$ the plane cutting the FS is very near one of the four-sided faces. Note that each of these faces is actually a spherical depression. The plane is chosen to pass between the bottom of the depression and the $z^{\prime}$ value where the cross section would break down into four small peaks.

For $z^{\prime}= \pm 1.4375 \AA^{-1}$ the $z^{\prime}$ plane is far enough out the $z^{\prime}$ axis that it catches only the four peaks on the second zone PS.

The cross section plots can also be overlaid to give a contour map effect of the second zone hole 

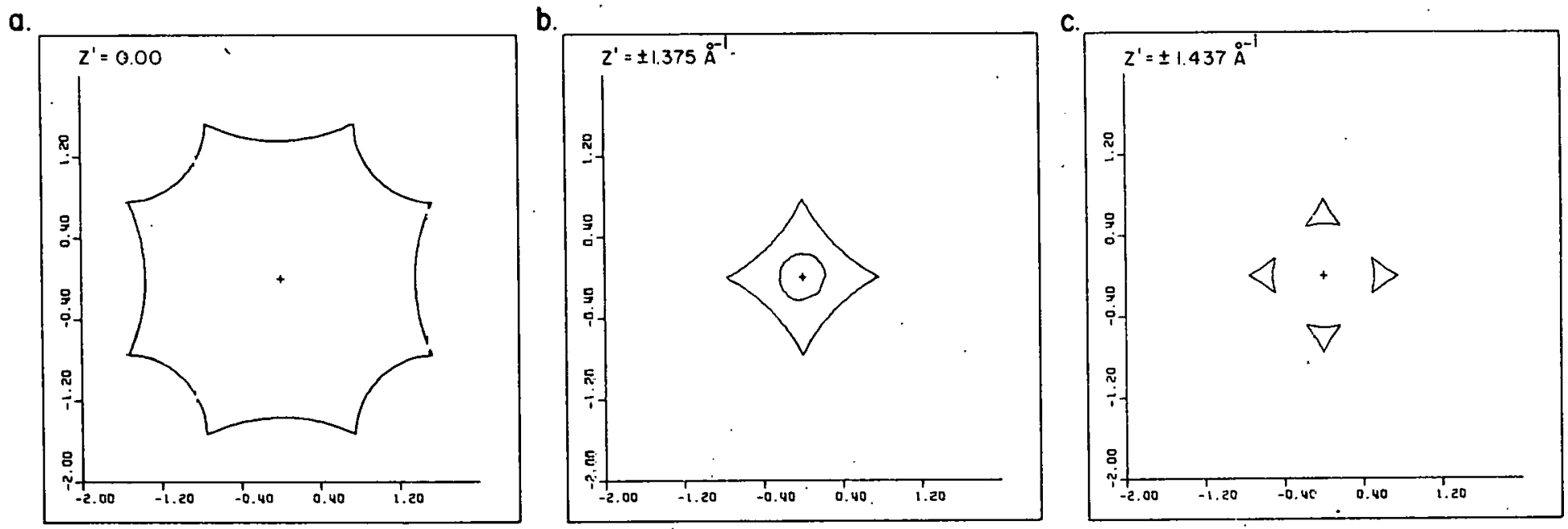

Figure 18. Three typical FS cross section outlines for the NFE aluminum second zone FS shown for a sample

with a [100] normal. The distances fron the zone center are a) $z^{\prime}=0.0$, b) $z^{\prime}= \pm 1.3750 \AA^{-1}$, and.c)

$z^{\prime}= \pm 1.4375 \AA-1$ 
surface as seen looking down the $z^{\prime}$ axis. Sample overlays are shown in Figures 19, 20, and 21 where the sample normals have been chosen as [100], [110], and [ 111$]$ respectively.

3) Now the cross section information for all the $z^{\prime}$ slices must be used to obtain caliper information for actual electron orbits. As noted earlier, electron orbits in $k$-space in a magnetic field are on surfaces of constant energy and in planes perpendicular to the magnetic field. For the problem under consijeration, the information on the constant energy surface has al ready been calculated. Now the direction of the magnetic field must be taken into account. Since information is desired for zalipers as a function of magnetic field angle for ranges of $45^{\circ}$ (for a [100] sample normal), $90^{\circ}$ (Eor a [110] sample normal), and $30^{\circ}$ (for a [111] sample normal) it is desirable to use an approach that will easily allow the angle of the magnetic field to be changed. This is most easily handled by a simple rotation of the coordinate system around the $z$ ' axis so the new $Y$ axis (Y") is parallel to the magnetic field. The new coordinate systel will be $x^{\prime \prime}, y^{\prime \prime}, z^{\prime \prime}$ where $z^{\prime \prime}=z^{\prime}=$ sample normal, $y^{\prime \prime}=$ direction of the magnetic field, and $x^{\prime \prime}$ takes its required position. In this new coordinate system, 


\section{AL SECOND ZONE FERMI SURFACE CROSS SECTION OVERLAY PLOT COMPUTER OUTPUT}

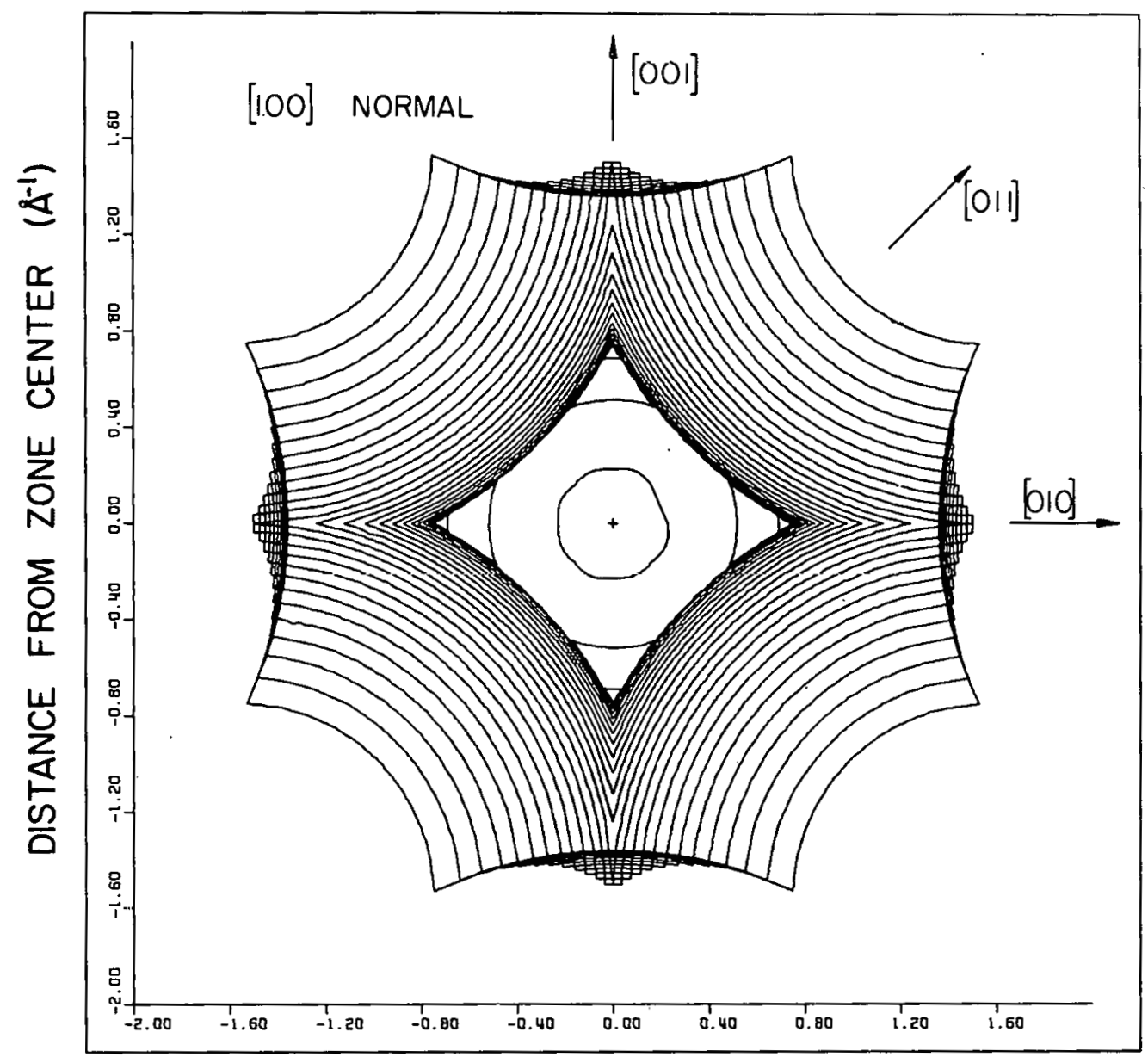

DISTANCE FROM ZONE CENTER $\left(\AA^{-1}\right)$

Figure 19. Cross section overlay plot for the aluminum NFE second zone FS for a [100] sample normal. Fross sections are shown for the plane through the center of the $B Z$ and for every increment of $z^{\prime}=0.0625 \AA^{-1}$ along the sample nornal direction. Major symetry directions are indicated 


\section{AL SECOND ZONE FERMI SURFACE CROSS SECTION OVERLAY PLOT COMPUTER OUTPUT}

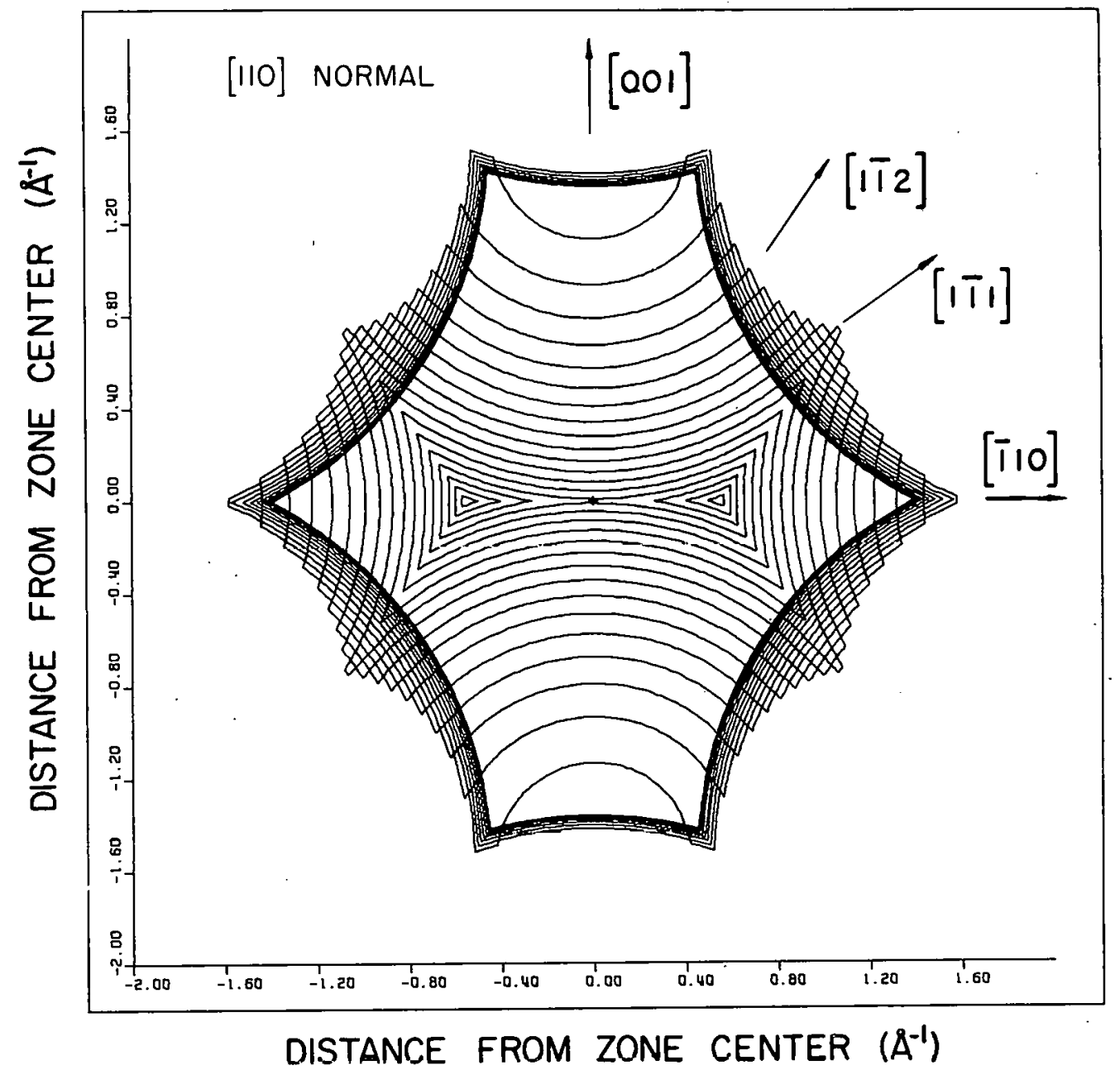

Figure 20. Cross section overlay plot for tha aluninun NFE second zone FS for a [110] sample normal. cross sections are shown for the plane through the center of the $\mathrm{Bz}$ and for every increment of $z^{\prime}=0.0625 \AA^{-1}$ along the sample nornal aicection. Major symetry directions are indicated 


\section{AL SECOND ZONE FERMI SURFACE CROSS SECTION OVERLAY PLOT COMPUTER OUTPUT}

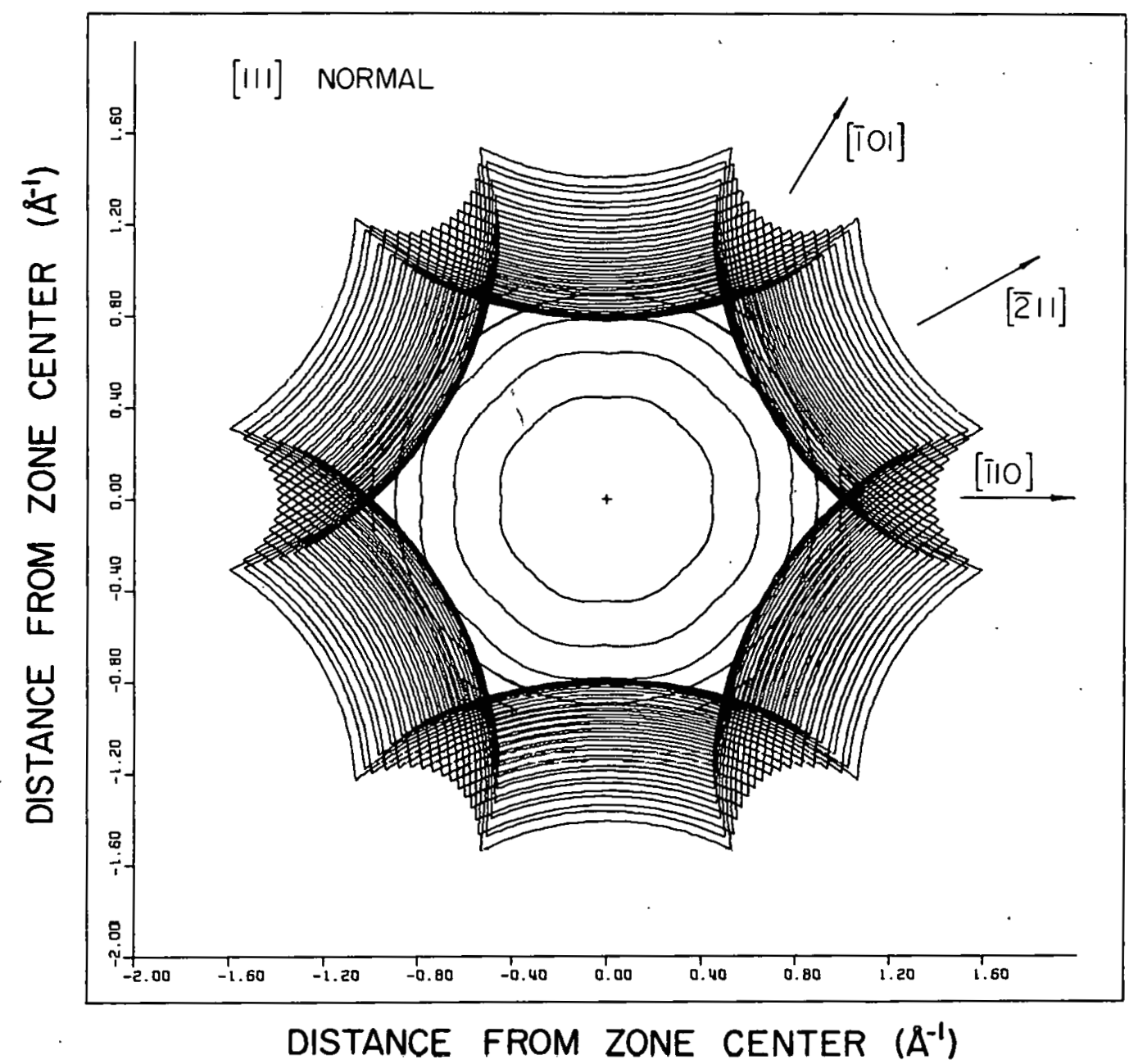

Figure 21. Cross section overlay plot for tha alumiau NFE second zone FS for a [111] sample normal. Cross sections are shown for the plane through the center of the $B Z$ and for every inzrement of $z^{\prime}=0.0625 \AA^{-1}$ along the sample normal direction. Major symetry 1 irections are indiated 
electron orbits in planes perpendicular to the magnetic field are simply in planes of constant $y^{\prime \prime}$.

In a constant $y^{\prime \prime}$ plane, points on an electron orbit can be found by evaluating the intersections of the $Y^{\prime \prime}$ plane with the PS cross sections on each $z^{\prime}$ plane as found in the second part of the calculation. After finding the intersections with all fs cross sections for the $57 \mathrm{z}$ ' slices, the resulting set of points describes the actual electron orbits in the $y^{\prime \prime}$ plane. Figure 22 illustrates a typical y" plane and the intersections found with the cross sections found previously. The resulting orbit is shown in Figure 23 for a [100] sample normal vith $\bar{H}$ at $15^{\circ}$ from the $x^{\prime}$ axis and $y^{n}=-0.4375 \AA^{-1}$. The caliper for a given orbit is then the largest value of $x "$ between any tro points on the orbit. (The use of $x^{\prime \prime}$ cesults from the fact that the projection of the real space trajectory on the $y^{\prime \prime}$ plane is the same shape as the $k$-space orbit but it is rotated by $90^{\circ}$ about the direction of the magnetic field, the y" axis. Therefore $x^{\prime \prime}$ in $k$-space will correspond to the actual $\Delta \bar{r}$ that will span the sample in real space parallel to the $z^{\prime \prime}$ axis.)

At this point the mechanics have been developed to find the orbit caliper, in a given plane perpendicular to the magnetic field. This procedure was then 


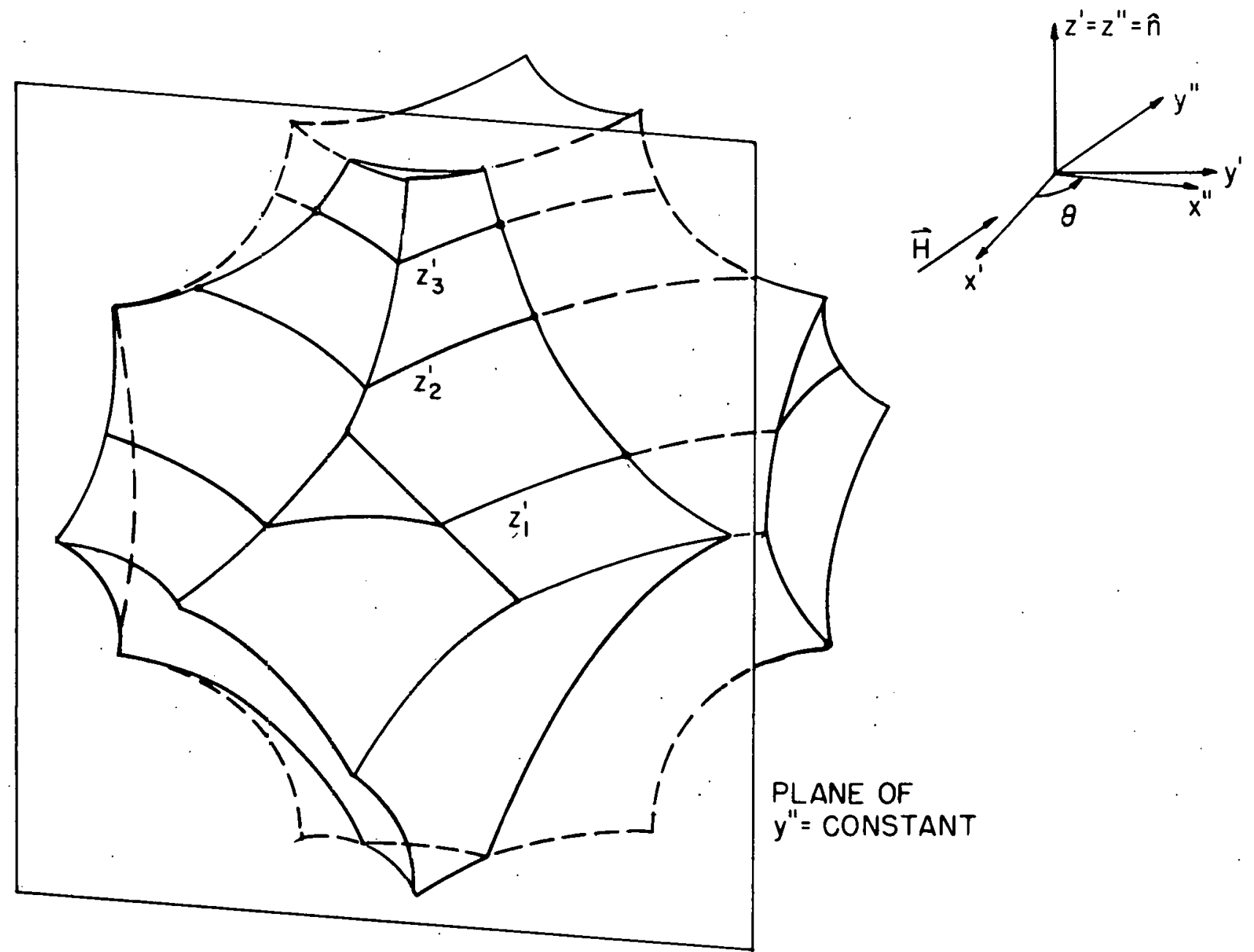

Figure 22, The NFE second $z$ one FS of aluminum is shown with the lines of intersection with thrae $z^{\prime}$ planes. The $y^{\prime \prime}$ plane is shown perpendicular to the applied magnetic field, $\vec{H}$. The resulting intersection with the FS indicates an eleztron orbit in $k$-space 


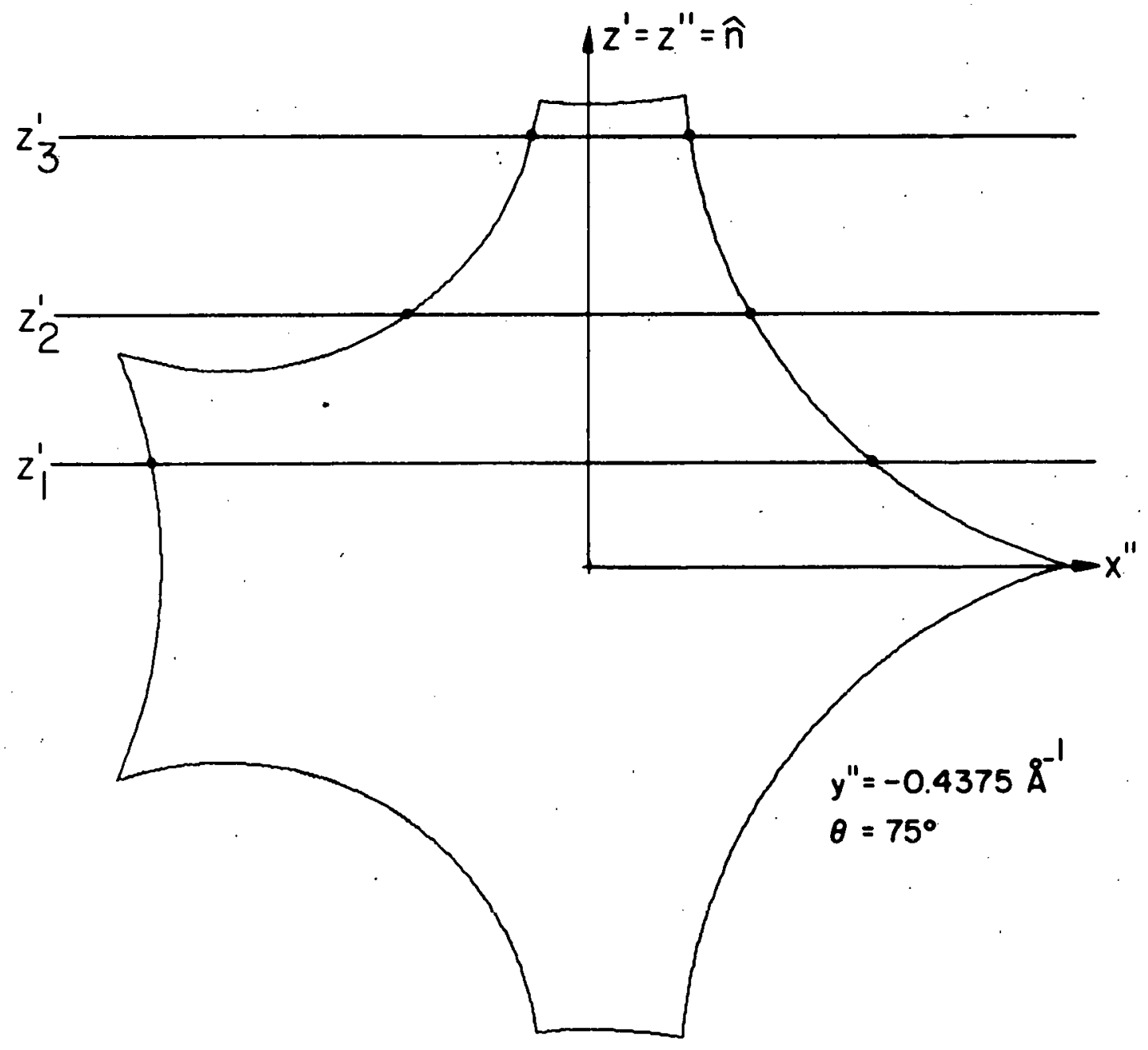

Pigure 23. An electron orbit is shown with its intarsections with three $z$ ' planes 
repeated for 113 equally-spaced planes of constant $y^{\prime \prime}$ beginning at the center of the Brillouin zone and advancing out the $+Y^{\prime \prime}$ axis. The range of $Y$ " was large enough to be sure that the last planes were beyond the second zone $\mathrm{FS}$ for an possible normal and magnetic field dicections.

The symmetry of the PS made it possible to determine the required calipers by examining only the $+y^{\prime \prime}$ axis.

4) The remaining probled is to relate these calipers to the intensities expected for RFSE signals. The magnitude of the contribution to the RFSE signal is related to the number of orbits with that caliper.

To calculate this the caliper range from $\Delta k=0$ to $\Delta k=4 h^{-1}$ vas broken into 128 bins of equal width. Then the caliper associated with each value of $y^{n}$ was examined to find the appropriate bin. The number in that bin was then incremented by one and the procedure was repeated for the next $y^{\prime \prime}$ slice. The end result was that each bin contained an integer representing the number of $y^{\prime \prime}$ slices that had orbit calipers uithin the range of that bin. This gives information concerning the relative strengths of contributions to RFSE signals for all calipers from $0 \AA^{-1}$ to $4 \AA^{-1}$. 
This information is valid for only one orientation of the magnetic field relative to the crystal axes. The procedures described in 3 and 4 are repeated for each angle desired between the magnetic field and the crystal axes. The information is then output in tabular ford. The vertical axis is the caliper, the horizontal axis is magnetic field angle, and the numbers in the table cepresents the number of orbits found in that range of the caliper. Figures 24,25, and 26 shoy the results for [100], [110], and [111] sample normals.

To aid interpretation, the tabular inforation can be used to obtain contour plots that show the symetry and angular trends of the expected datz. This is shown in Figures 27, 28, and 29.

The origins of the various lines on the contour plots can be examined by using an option built into the program. It is possible to change one data card and have the caliper for each $y^{\prime \prime}$ value printed out for each angle examined, so that for each angle a list of 113 calipers is printed. These can then be examined to see from which ranges of y" a signal is resulting. These ranges of $I^{\prime \prime}$ can then be used rith the cross section overlay plots to locate the orbits contributing to the signal. Consider a sample with a [100] normal axis. The signal intensity contour plot in Figure 27 for $\theta=34^{\circ}$ shows relatively strong signals at Permi surface calipers of 


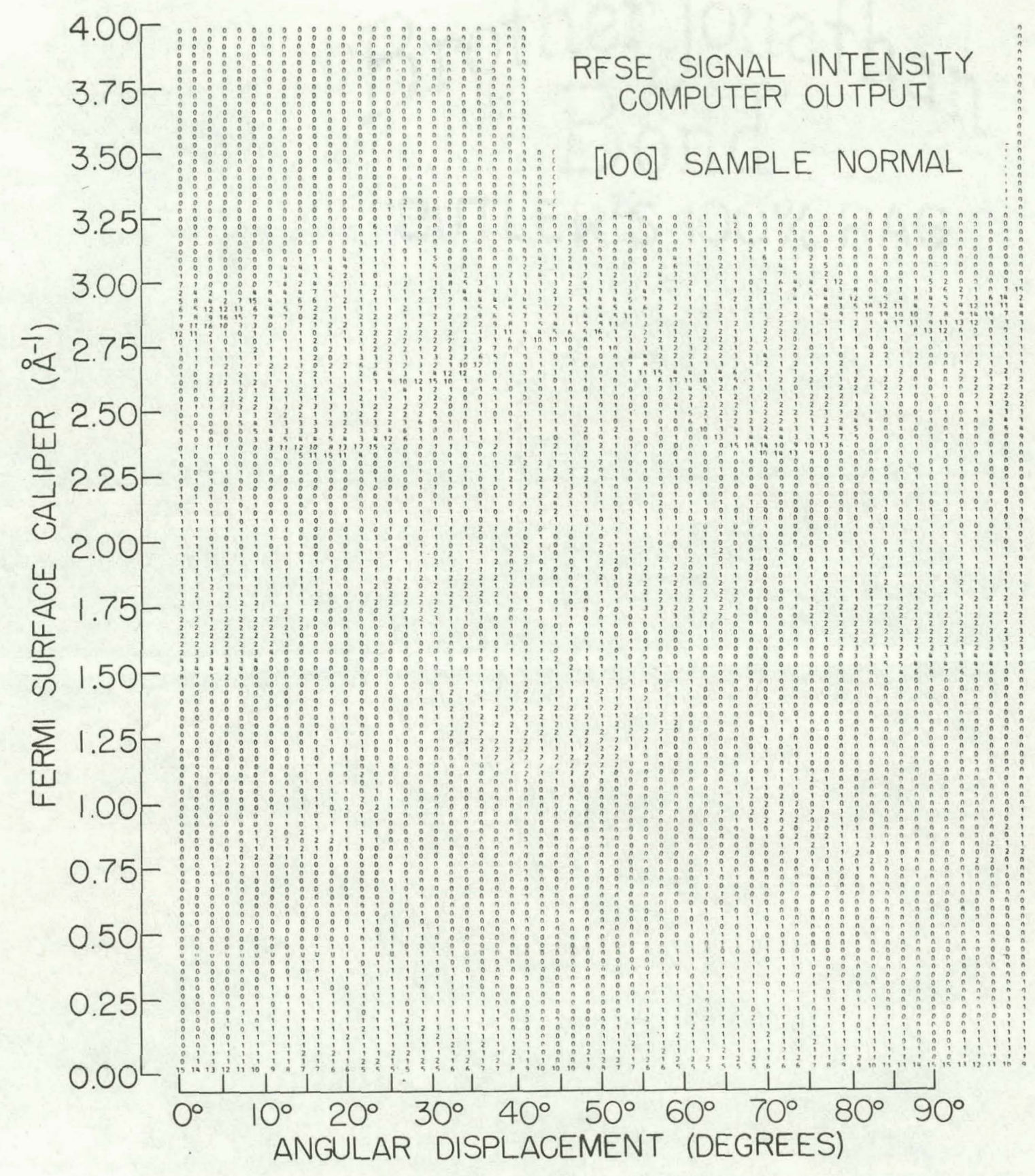

Figure 24. RFSE signal intensity for NPE second zone FS of aluminum for a [100] sample normal. Numerical values in the table indicate relative intensities. Major crystal axes and their locations are $[010]$ at $00,[011]$ at $45^{\circ}$, and [001] at $90^{\circ}$ 


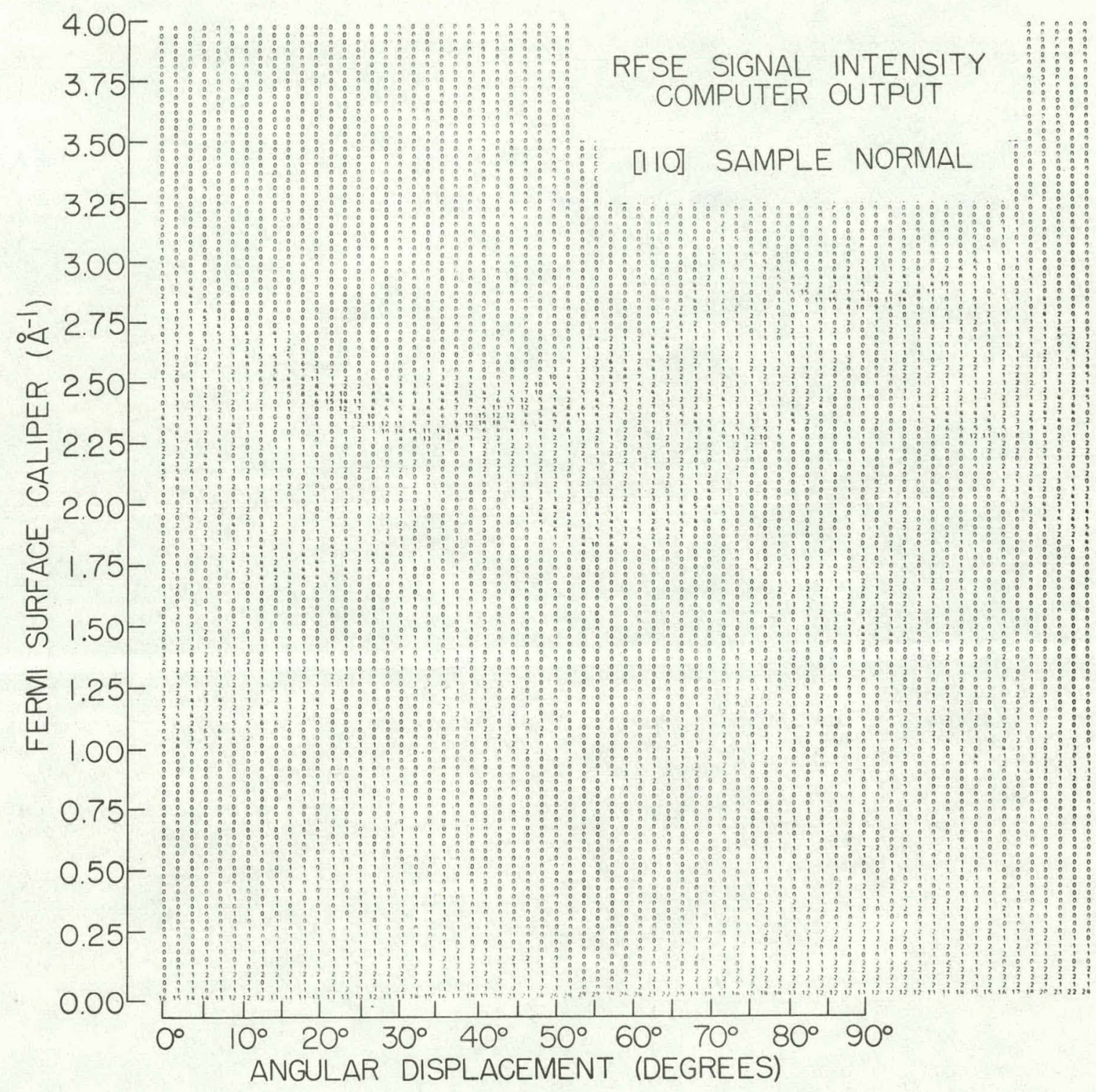

Figure 25. RFSE signal intensily fur NFE second zone FS of aluminum for a [110] sample normal. Numerical values in the table indicate relative intensities. Major crystal axes and their locations are $[1 \overline{10}]$ at $0 \circ,[111]$ at $35^{\circ},[1 \overline{1} 2]$ at $55^{\circ}$, and [001] at $90^{\circ}$ 


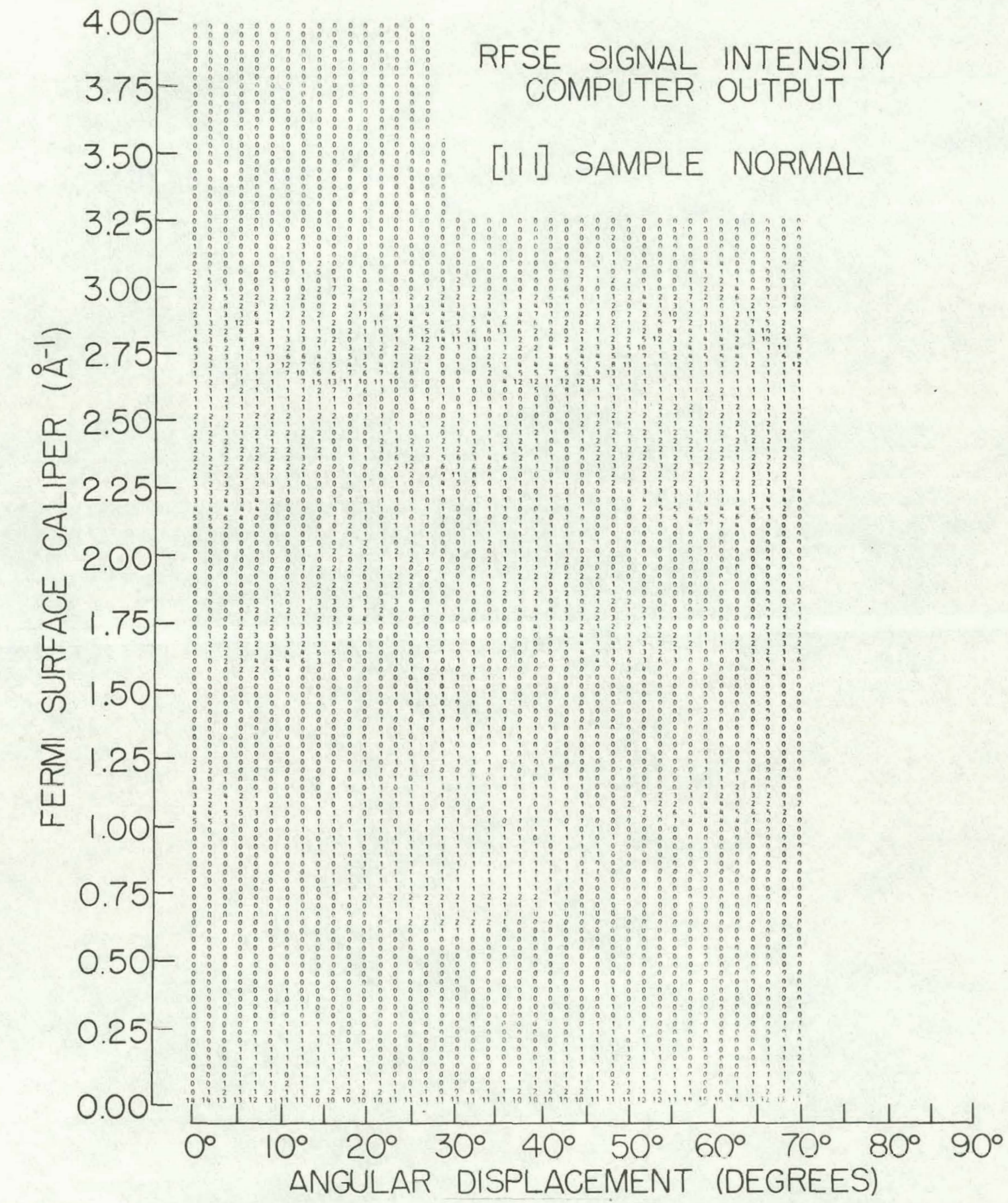

Figure 26. RFSE signal intensity for NFE second zone FS of aluminum for a [111] sample normal. Numerical values in the table indicate relative intensities. Major crystal axes and their locations are [ 101 ] at $00,[211]$ at $30^{\circ}$, and [110] at $60^{\circ}$ 


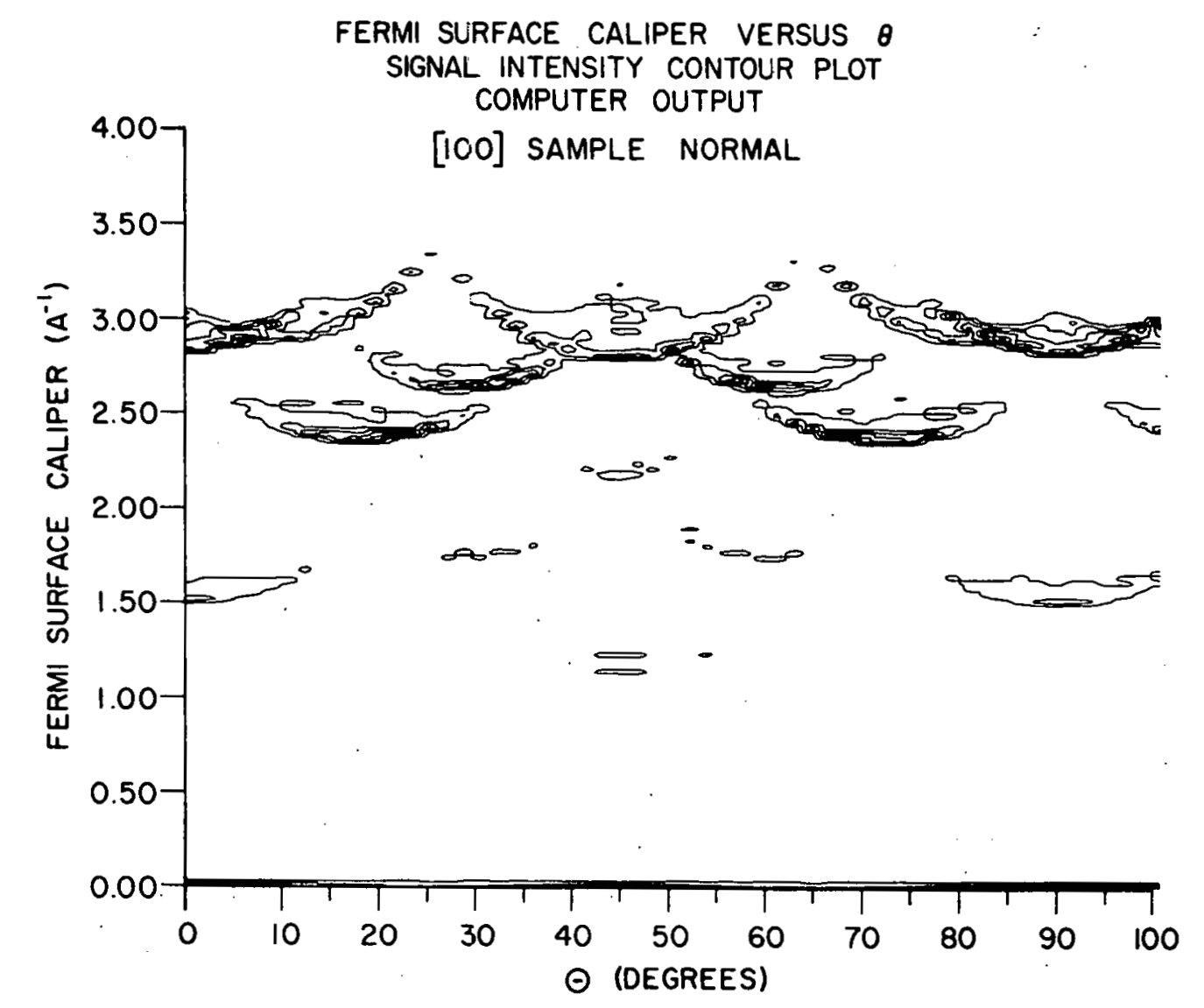

Pigure 27. RFSE signal intensity contours for NFE second zone PS of aluminum for a [100] sample normal based on numerical yalues shown in pigure 24. The initial contour is drawn at an intensity of 2.5 and successive contours at intensity intervals of 3. Major crystal axes and their locations are $[010]$ at $00,[011]$ at $45^{\circ}$, and [001] at $90^{\circ}$ 


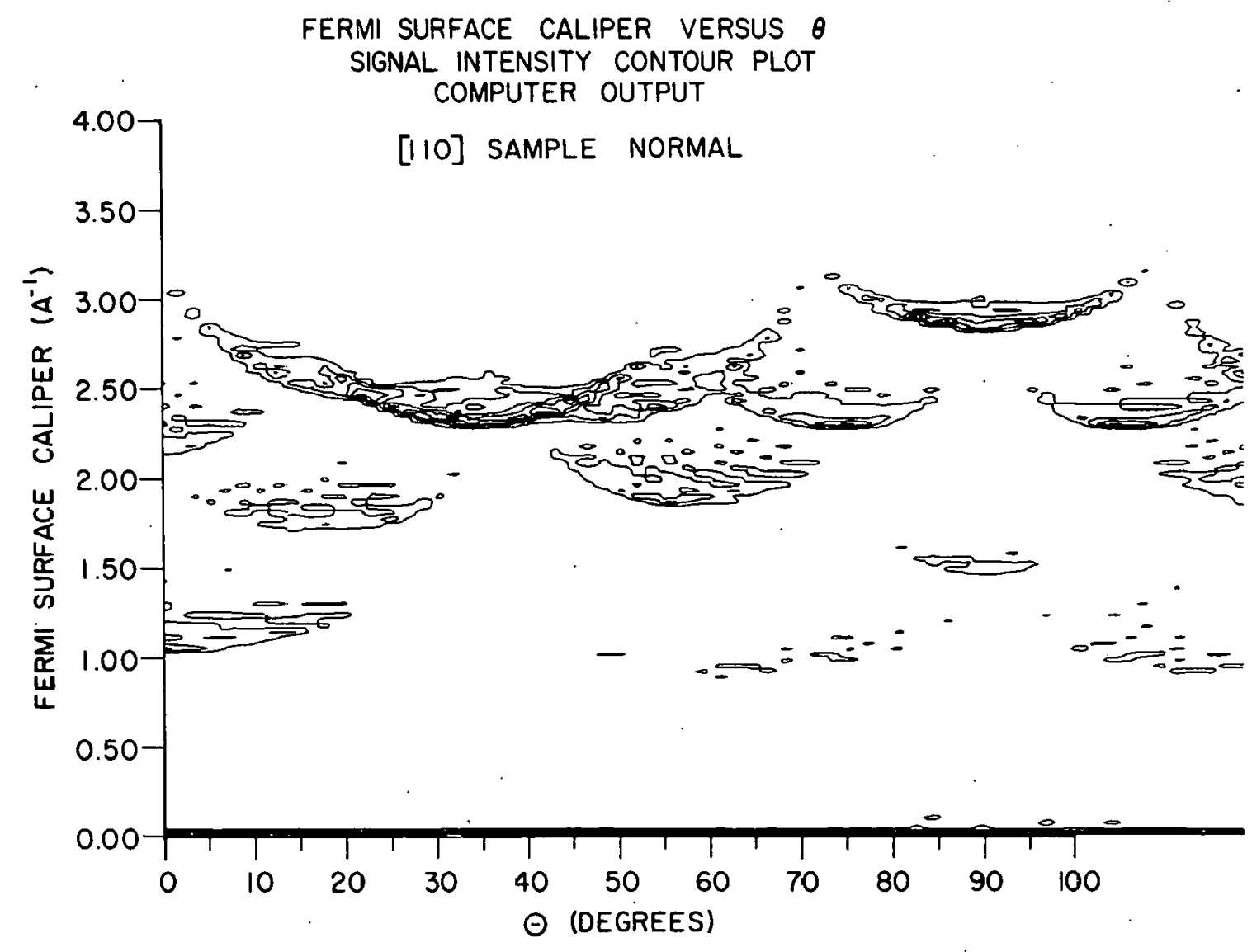

Figure 28. BPSE signal intensity contours for NFE second zone FS of aluminum for a [110] sample noral based on numerical values shown in Figure 25. The initial contour is drawn at an intensity of 2.5 and successive contours at intensity intervals of 3 . Major crystal axes and the ir locations are [1 10 ] at $00,[111]$ at $35^{\circ},[112]$ at $55^{\circ}$, and $[001]$ at $90^{\circ}$ 


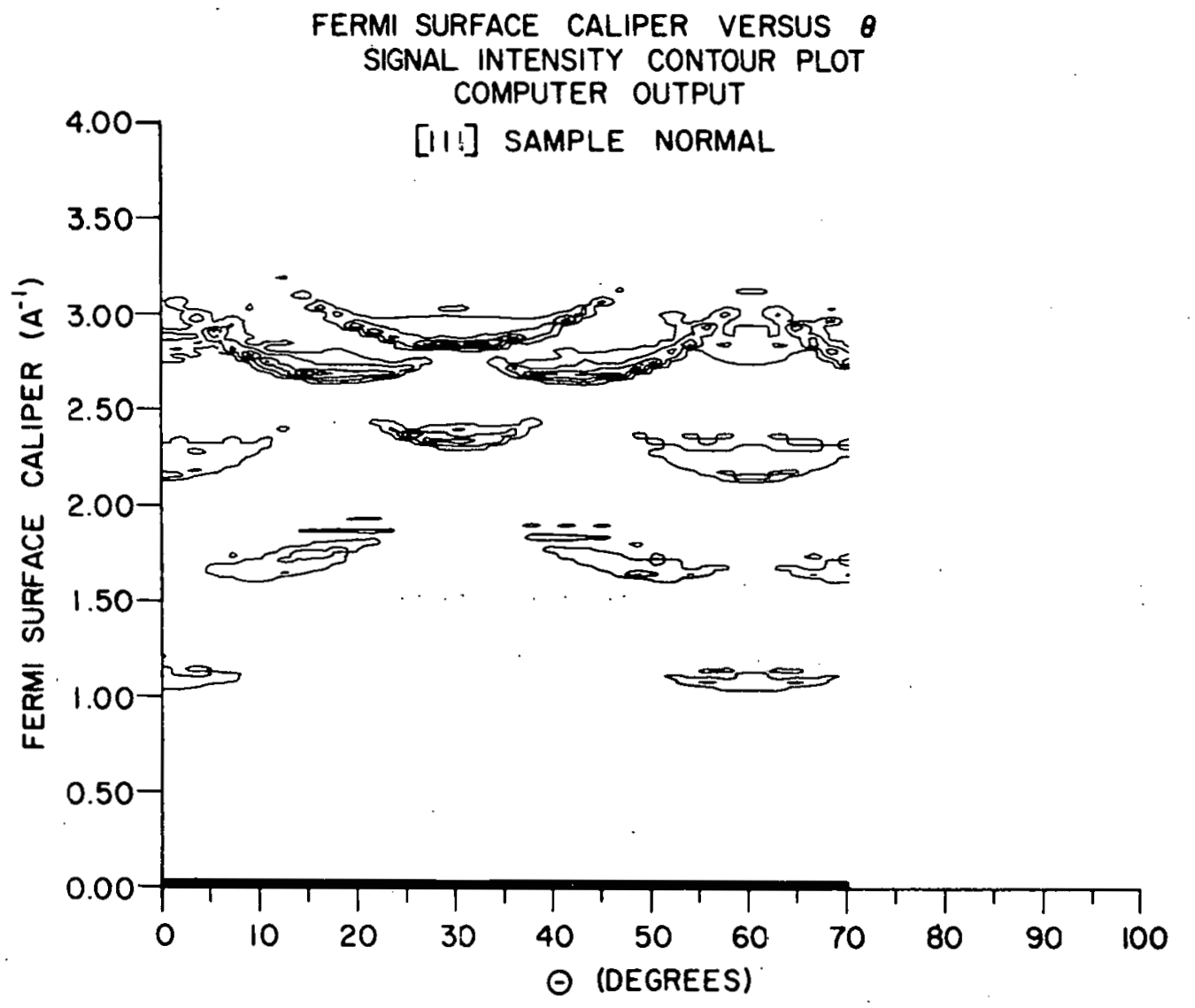

Pigure 29. RFSE signal intensity contours for NFE second zone PS of aluminum for a [111] sample normal based on numerical values shown in Figure 26. The initial contour is drawn at an intensity of 2.5 and successive contours at intensity intervals of 3.-Major crystal axes and their locations are $[\overline{1} 01]$ at $00,[\overline{2} 11]$ at 300 , and [ 110$]$ at $60^{\circ}$ 
about 2.70 $\AA^{-1}$ and about 3.00 $\AA^{-1}$. Examination of the computar results indicates that the calipers near $2.70 \%-1$ arise from orbits crossing the Fermi surface in the region marked "A" in Figure 30. Similarly the or bits with calipers near 3.00\%-1 are indicated by the region marked "B" in the same figure. Due to the symmetry of the FS, identical orbits also exist on the other side of the origin from those shown in the figure. This type of orbit location is valuable because it allows examination of the actual orbits contributing to a signal. This will be useful when signals from actual data are compared to these NFE model predictions.

This calculation of calipers is not without limitations. The FS model used does not include rounding effects on the edges and tips of the rermi surface which result from interactions not included in the nearly free electron model. This will cause all calipers to be too large; hovever, shapes and relative locations seen on the tabular output and contour plots should still be very helpful.

The calculation does not include any method to handle electron drift velocities that might be helpful in the identification of signals due to non-central orbits. This means that the program cannot be used to examine the consequences of tilting the sample's surface relative to the magnetic field as done for potassium by peercy et al. (143). 


\section{AL SECOND ZONE FERMI SURFACE CROSS SECTION OVERLAY PLOT COMPUTER OUTPUT}

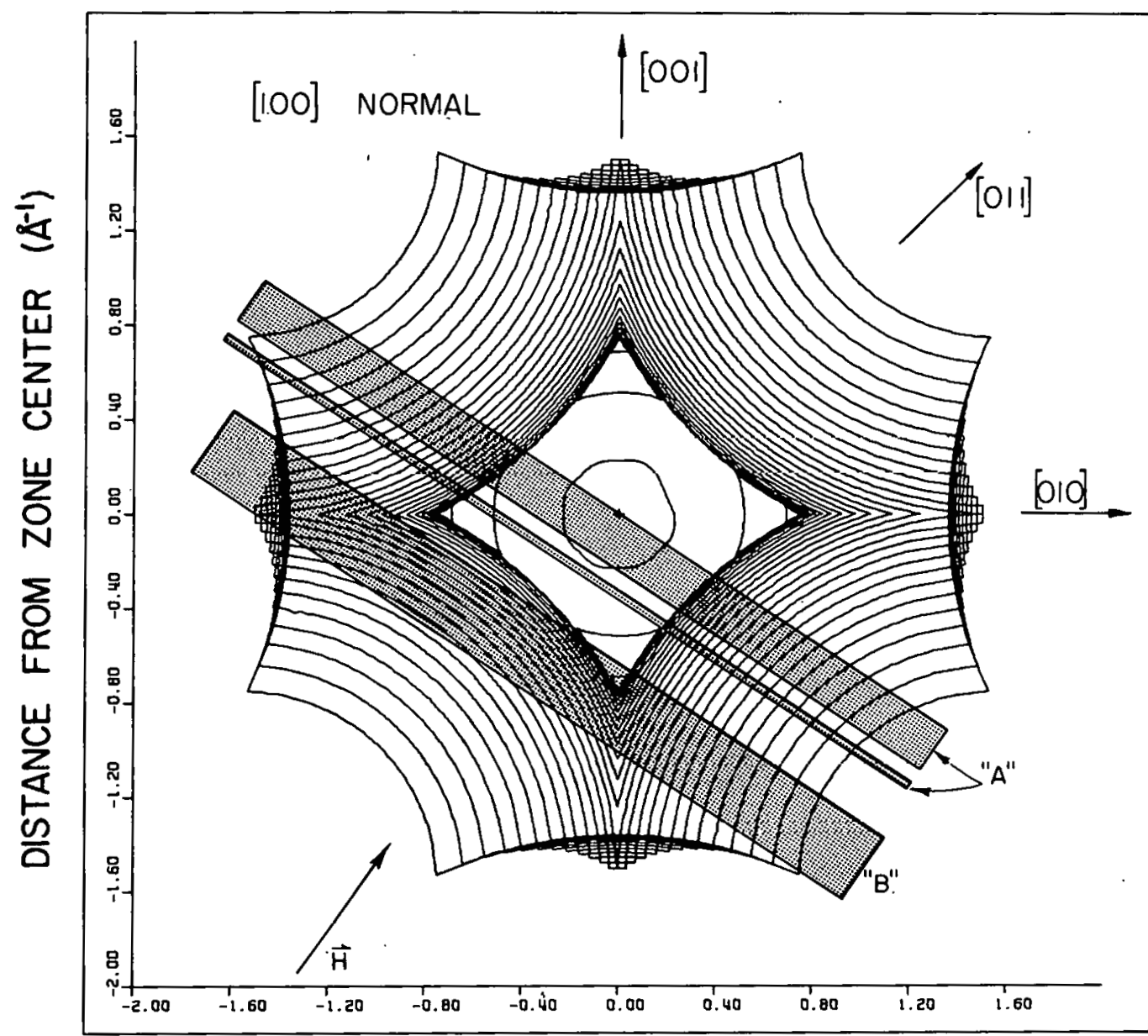

DISTANCE FROM ZONE CENTER $\left(\AA^{-1}\right)$

Figure 30. The cross section overlay plot is shown for the NFE second zone FS of aluminum. The magnetic field is indicated. The shaded ragions indicate electron orbits that contribute to FS calipers near $2.70 \AA^{-1}$ and $3.00 \AA^{-1}$ that ace labeled "A" and "B" raspectively 
The calculation examines only full orbits around the PS. It does not yield results relating to the type of partial orbits seen by Gantmakher and Krylov (144) in indium, as dis-. cussed earlier.

These limitations should not have too much effect on the usefulness of the caliper results. The trends in calipers as the magnet angle is varied should be very useful. Comparisons can also be made ith experimental data using relative magnitudes of calipers at different angles.

The calculation of signal intensities does not take into consideration the shape of the electron trajectory near the sample's surface. Thus the effectiveness of the trajectory as it interacts with the $f$ field in the skin depth is not considered. Electrons moving nearly parallel to the surface interact with the rf electric field effectively and the longer their path remains in the skin depth, the more they will interact with the field. Trajectories that simply jut into the skin depth and then quickly return to the bulk of the sample are much less effective in their interaction with the electric field. Figure 31 contains examples of effective and ineffective orbits.

For this reason, small changes in intensity as indicated in calculated results may be open to question but large changes are probably a good indication of wat to expect in the experimental data. If further information is needed in 


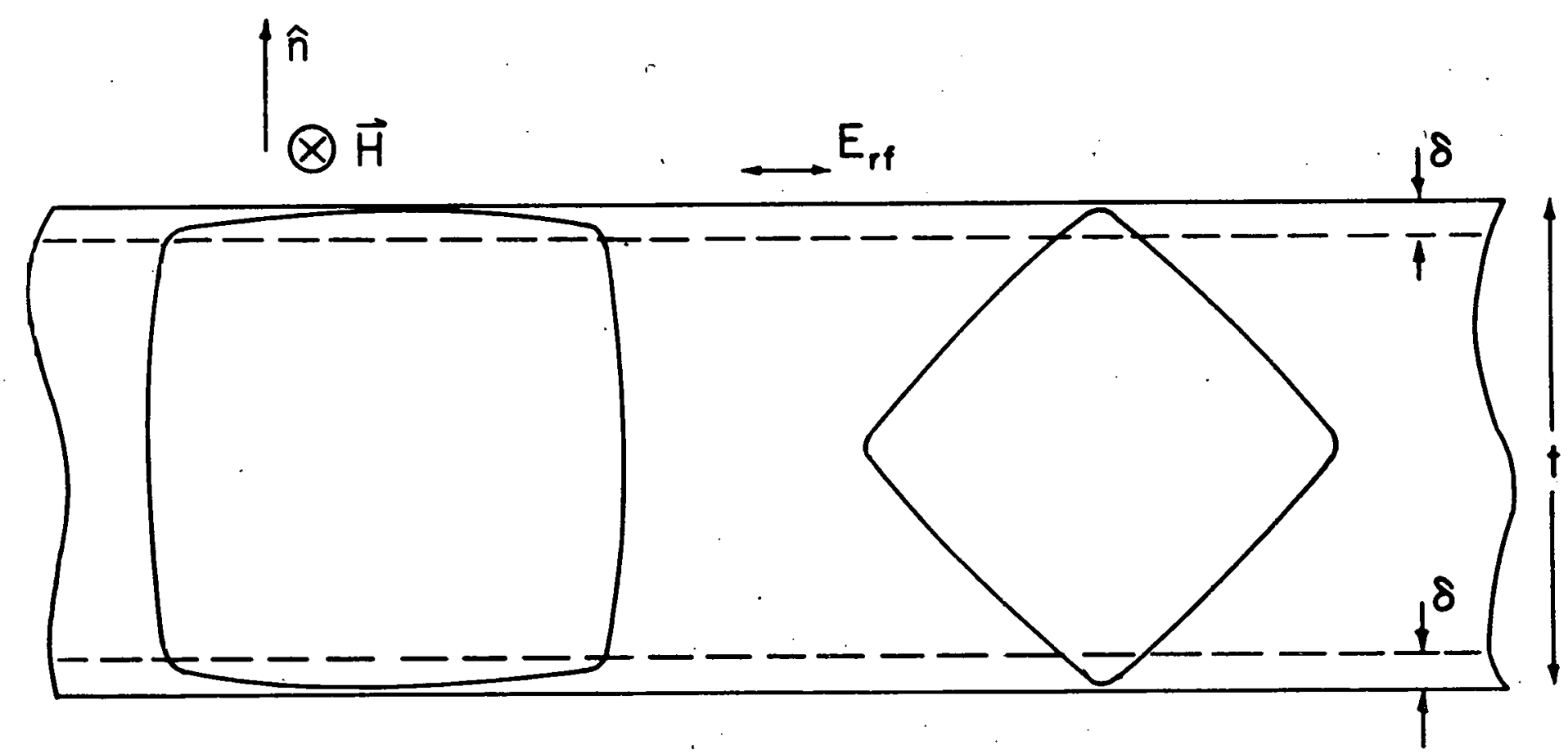

Pigure 31. Two electron trajectories are shown spaning the sample. The trajectory on the left spends 1 considerable amount of time exposed to the $x f$ fiel ls in the skin depth and is an effective trajectory. The trajectory on the right spends very little time within the skin depth and is an ineffective trajectory 
this regard, the program can be used (as discussed earlier) to find the actual electron orbits (and trajectories) contributing to the calipers in question. Then the effectiveness of these trajectories can be examined visually to determine if one orbit is expected to be much more effective in contributing to the rf size effect. If so, the calculated intensity results can be expected to shou poor correlation to intensities seen experimentally.

This program was written in the $\mathrm{PL} / 1$ programing language to allow the ase of based variables (145). Then using these variables, storage space in the computer is allocated as needed in the calculation. This was very useful in this case because the quantity of information calculated and stored for the cross sections in the beginning of the calculation depended greatly on the sample normal being considered.

Not only quantity but also the organization of this information depended greatly on the normal chosen. This can easily be seen by noting that different $z^{\prime}$ slices have different numbers of orbits (from zero to six) and orbits have different number of sides (from three to ten). If fixed storage were used, then enough vould be required for six orbits vith ten sides on each z' slice. This vould be extremely wasteful. It would greatly increase runing costs and it would also increase turn-around time for the calcula- 
tion.

By using $P L / 1$, the storage for all this information is exactly the size needed. PL/1 also allows the use of linked lists. This is a convenient methol of bookkeeping that allows organization of all the stored items so they can be systematically retrieved for further calculations. 


\section{THE ALUMINOM EXPERTMENT}

\section{A. Apparatus}

The RFSE is observed by studying anomalies in the surface impedance of a flat metal sample placed in a static magnetic field. The surface impedance has real and imaginary parts, the surface resistance and surface reactance respectively, and the anomalies can be studied by examining either portion of the impedance. The method of observation used in this investigation utilizes changes in the surface reactance by placing the sample in the system in such a manner that. changes in the surface impedance can be readily detected using a standard rf communications receiver. A block diagram of the apparatus is shown in Pigure 32 .

The sample is placed inside a coil that serves as the inductance portion of a tank circuit used to determine the frequency of a variable frequency oscillator. The zoil and sample are then lowered into the liquid helium dewar and 10cated at the center of the pole gap of an electronagnet. Phosphor-bronze springs are used to keep the sample holder assembly centered in the dewar and to decrease vibrations. The impedance of the coil-sample combination reflects both the capacitance and inductance of the coil and the surface impedance of the sample (S1). Theretore, when surface impetance anomalies occur, they are reflected in the impedance of the tank circuit and therefore in amplitude and frequency 


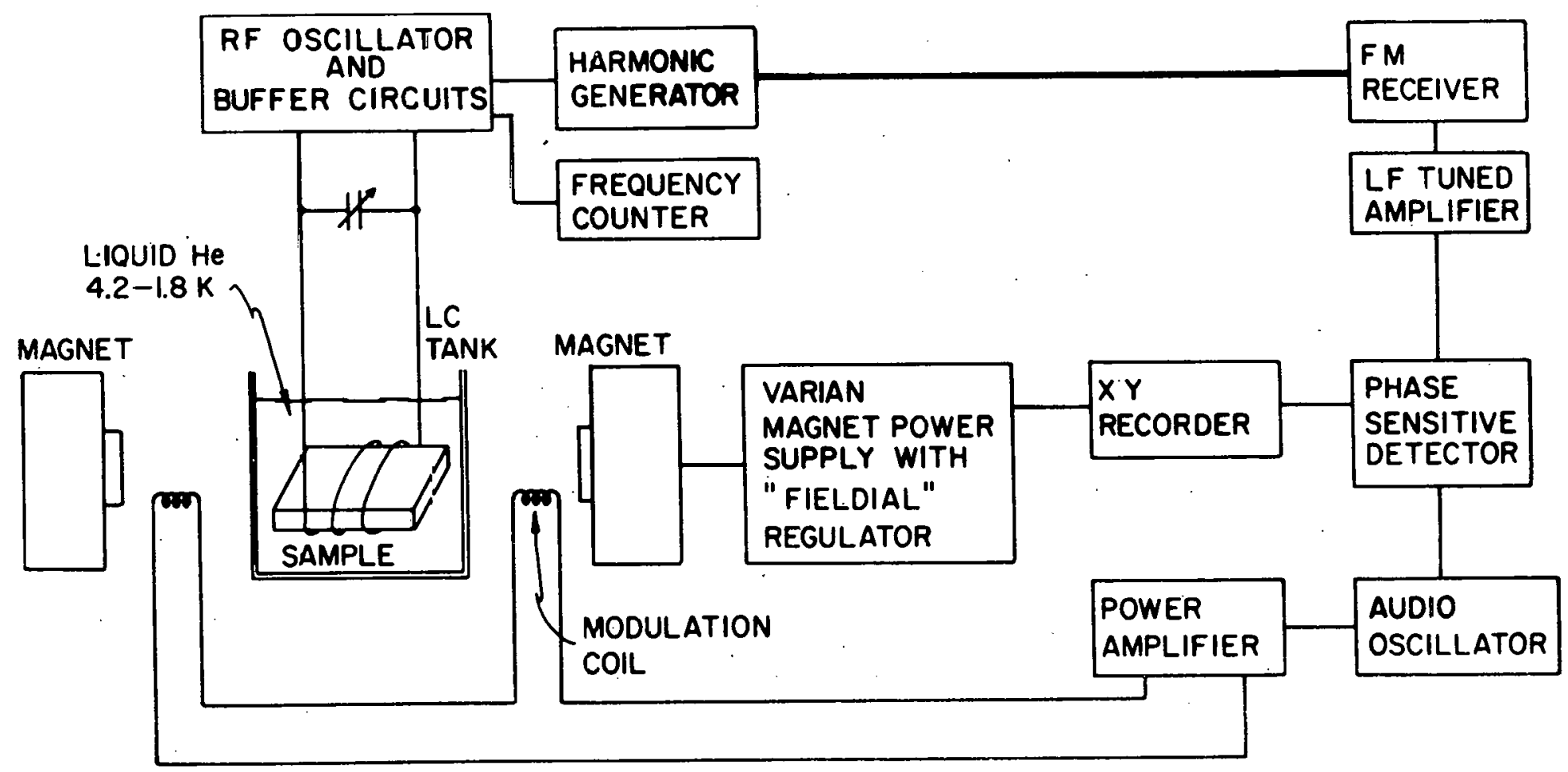

Pigure 32. Block diagram of the apparatus used in this RFSE investigation. The resulting $X-Y$ recorder trace is df/dH verses $H$ 
changes of the signal in the oscillator.

In all discussions to this point the applied magnetic field has been called a static field. In fact the field only needs to remain constant long enough for an electron to complete its mean free path ithout its trajectory size changing due to changes in the magnetic field. Since a typical time for an electron between scatterings is about 10-14 seconds, slow changes in the field will not invalidate any of the principles already mentioned. This result makes it possible to sweep the magnetic field as a function of time and to use a low frequency modulation field which will then facilitate the use of lock-in detection methods for the signal.

Consider the sample and coil subject to a fixed field $H$ plus a small modulation field given by $H_{1} \cos \omega_{M} t$. The frequency of the oscillator can then be written as

$$
f(H)=f_{0}(H)+(d f / d A) H_{1} \cos \omega_{m} t+\text { higher order terms in } I_{1}(21)
$$
where $(\mathrm{df} / \mathrm{dH})$ reflects the dependence of the coil-sample impedance on RPSE resonances and magnetoresistance effects in the sample. Cleveland (51) has shorn that if the coil has a large quality factor, $Q$, then the value of (df/dH) is direct1y proportional to the derivative of the surface reactance with respect to the magnetic field. Prom this it is seen that the modulation field leads to frequency modulation ( $f m$ ) of the oscillator frequency with a modulation amplitude of (df $/ \mathrm{dH}) \mathrm{H}_{1}$. 
The fm signal from the oscillator is then clipped to generate harmonics. Consider the n-th harmonic of the frequency $\mathrm{f}(\mathrm{B})$.

$n-t h$ harmonic $=(n+1) f(H)$

$$
=(n+1) f_{0}(H)+(n+1)(d E / d H) H_{1} \cos \omega_{M} t+h \cdot 0 \cdot t \text {. }
$$

where h.o.t. refers to higher order terms. Here it is seen that the modulation amplitude is now given by $(n+1)(d f / d H) H_{1}$ which is enhanced by a factor of $(n+1)$ over the value seen in the fundamental produced by the oscillator. Thus the signal to noise ratio can be improved by examining the harmonics rather than the fundamental.

The clipper output is detected by an fm commications receiver which is tuned to an integer multiple of the fundamental frequency produced by the oscillator. The output taken from the receiver is chosen to yield information on the modulation amplitude of the input fm signal. This output is directly proportional to (df/dH).

The receiver output is processed by a tuned amplifier and a lock-in detector (IID) to allow the small signal from the modulation amplitude to be recovered. The LID reference signal is derived from the same oscillator feeding the field modulation coils. The LID output is applied to the Y-axis on an $X-Y$ recorder.

The voltage supplied to the $X-a x i s$ of the $X-Y$ recorder is taken directly from the magnet pover supply which provides 
a voltage directly proportional to the value of the magnetic field relative to the end points selected for the field sweap being used. The resulting traces on the $X-Y$ recorder are then plots of ( $f(d H)$ versus the magnetic field value. The individual components represented in the apparatus block diagram will now be discussed and circuit diagrams are given for items designed and constructed during this investigation.

The oscillators and clipper-follower circuits were designed and built for this investigation. The circuits were fabricated on double-sided printed circuit boards with one side serving as a ground plane. Four oscillators ware built to cover the frequency range from $3 \mathrm{~Hz}$ to $37 \mathrm{MHz}$. The oscillator circuit design $(146)$ is shown in Figure 33 and the component values are found in Table 1 . The inductance $\mathrm{L}$ represents the coil containing the sample located in the liquid helium bath. The oscillators were \#0,\#1, $\# 2$, and \#3 with their frequencies decreasing as their assigned number increased. Oscillators in adjacent frequency ranges overlapped on each end. The exact frequency range of each oscillator for a given sample holder vas determined by the area, number of turns, and the wire size used for the sample holder coil. 


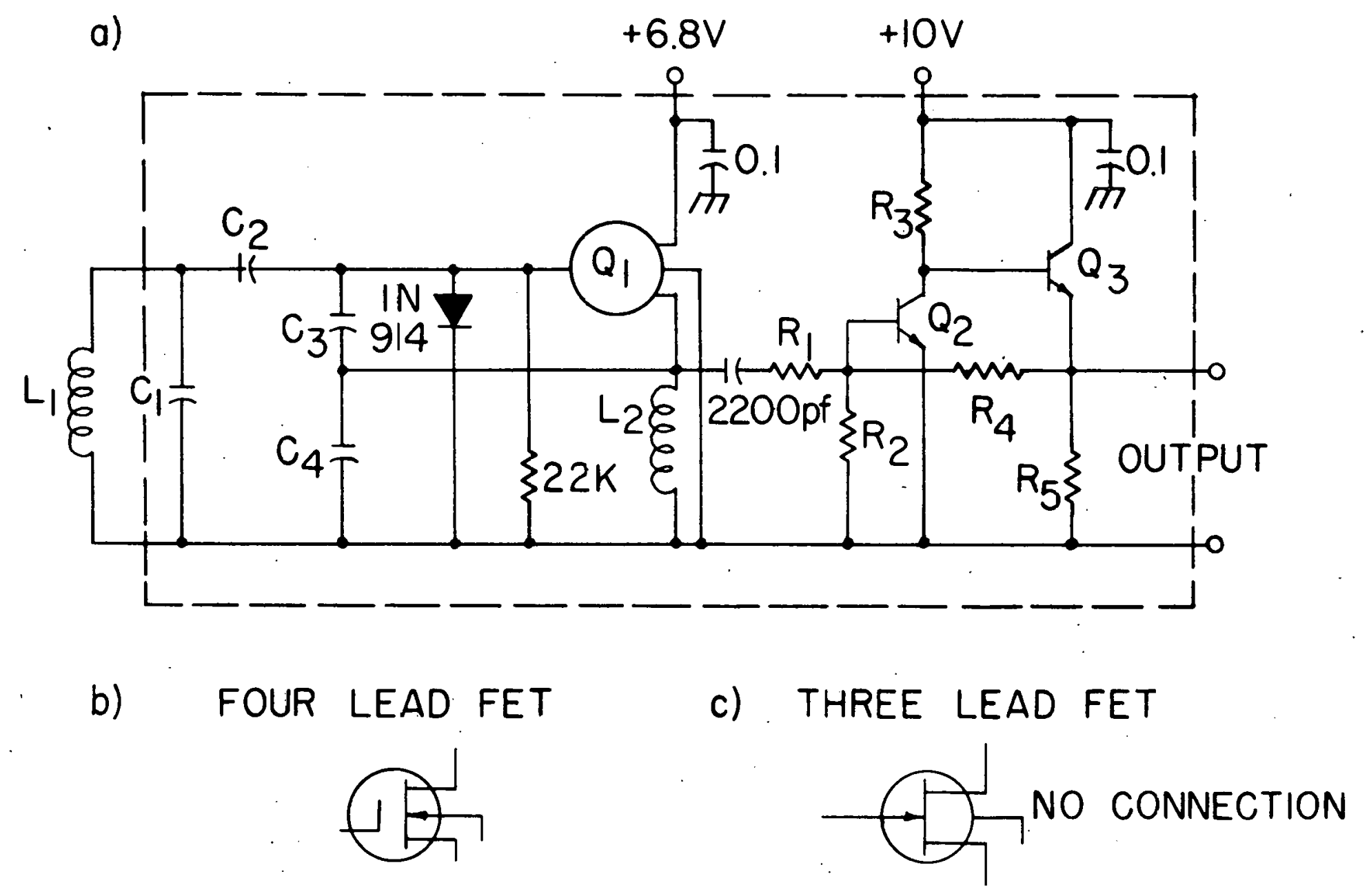

Pigure 33. Circuit diagram for transistor oscillators used in this RFSE investigation. Component values are listeł in Table 1. Capacitance values shown on the diagram are microfarads unless indicated otherwise 
Table 1. Components used in oscillators

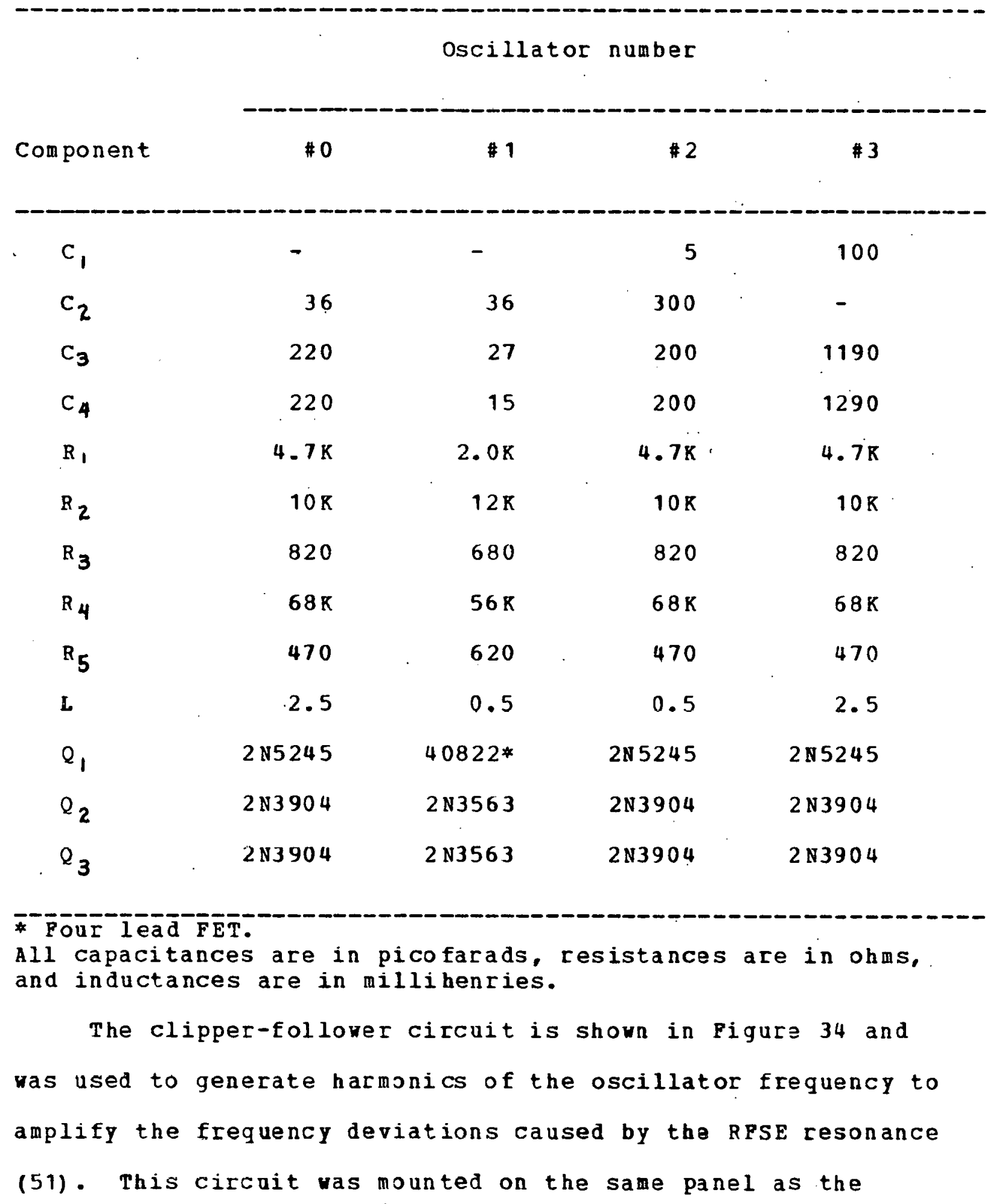




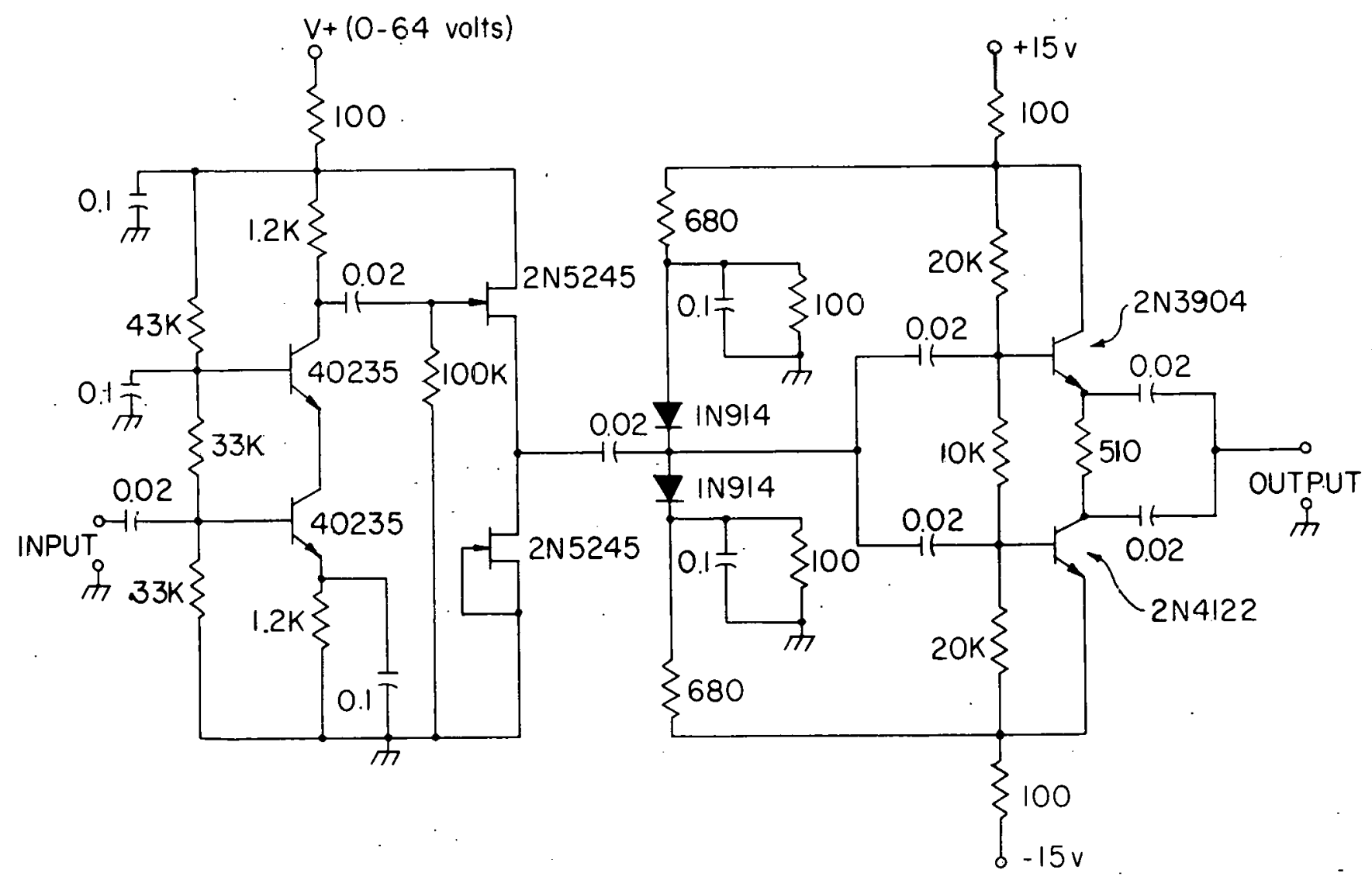

Figure 34. Circuit diagram for the clipper-follower used in this RFSE investigation. Resistances are in ohms. capasitances are in mizrofarads 
oscillators and coaxial cables were used for all

interconnections to minimize rf signal losses.

The adjustable $Q$ (quality factor) tuned amplifier was used to increase the signal level and reduce noise at frequencies other than the reference frequency. The design (147) allowed adjustment of both the $Q$ of the filter and the center frequency. Buffer amplifiers vere required on both the input and output to aroid a detuning of the circuit by the adjacent circuitry. The circuits used are shown in Figure 35. Initial data were taken using Fairchild uA741 operational amplifiers in the circuit. The signal to noise ratio was later improved when Precision Monolithics opera-. tional amplifiers (number Mono $O P-05 \mathrm{CJ}$ ) were used in the circuit. These two different devices are pin compatible so no circuit changes were required to facilitate the conversion.

The receiver used was an Eddystone model 770R (MKII) communications receiver ith a frequency cange of $19 \mathrm{MHz}$ to 165 MHz. The LID was an Electronics, Missiles, and Communications, Inc. model R JB lock-in amplifier.

A six-inch electromagnet made by Varian associates was used to provide the "static" magnetic field. The magnet was mounted on a rotating table marked in $1^{\circ}$ intervals which allowed rotation of the magnetic field with respect to the fixed crystal. The pover supply, a "Pieldial" Mark I, was 
a)

TUNED AMPLIFIER

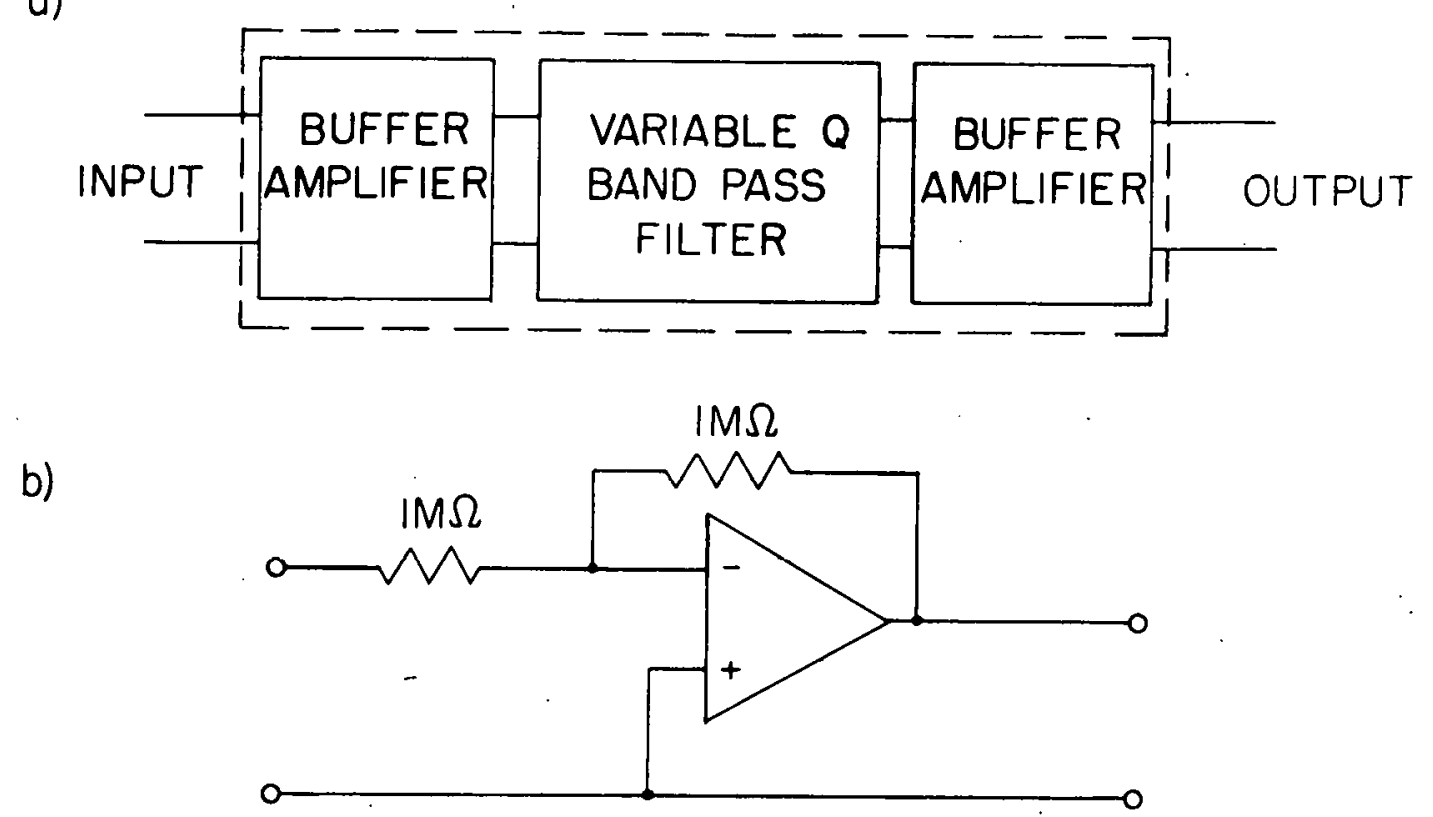

c)

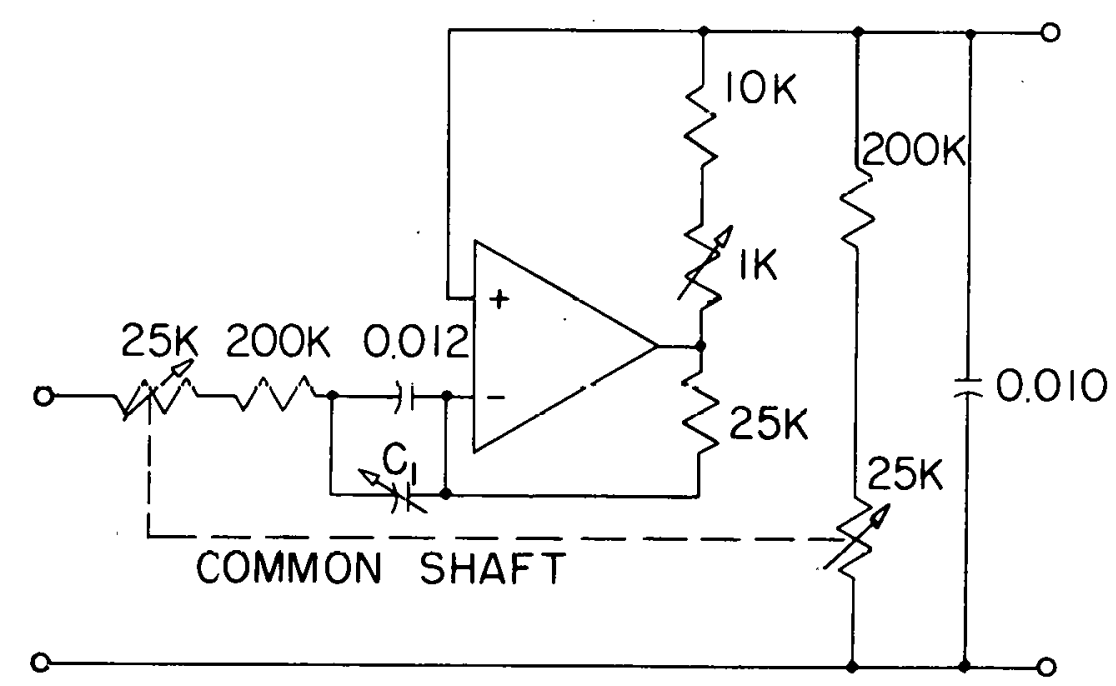

a) Block diagram of the adjustable $Q, 80 \mathrm{~Hz}$ tuned amplifier used in this investigation. b) circuit diagram for the buffer amplifier stages. c) Circuit diagran for the band pass filter stage. Double-ganged $25 \mathrm{~K}$ potentioneters adjust the center frequency and the $1 \mathrm{k}$ potentiometer adjusts the Q. C, is a 20 to 125 pf capacitor. other capacitances are in microfarads 
used to sweep the magnetic field at a rate that was constant in time (i.e. dH/dt could be chosen and would remain constant during a sueepl.

The low frequency signal used to supply the LID reference channel and the modulation field was supplied by a Hewlett-Packard model $204 \mathrm{C}$ solid state oscillator. The exazt frequency used ras chosen to match the frequency of the IID reference filters. The center frequency of the tuned amplifier was then adjusted to the same frequency.

The modulation field was obtained by amplifying the reference signal and applying the resultant voltage to two wire coils wound around the poles of the varian magnet. The amplifier used to drive the two coils was a single channel Heathkit, model AA-23.

During this investigation two modulation frequencies were examined. All the data to be discussed were taken with $80 \mathrm{~Hz}$ modulation. The effect of a lower frequency on. the signal to noise ratio was examined by the use of a $42 \mathrm{kz}$ signal and also a $42 \mathrm{~Hz}$ tuned amplifier. No improvement was observed so the system was returned to operation at $80 \mathrm{~Hz}$. The rf oscillator frequencies vere measured using a Monsanto model 110A, programable counter-timer. When the magnetic field was constant, the oscillators were stable to six significant figures based on a 100 millisecond counting period. 
The sensitivity of the apparatus allowed the observation of four NGR signals. The strongest was the NMR due to protons in the GE 7031 varnish used on the oscillator coil. NMR signals vere also seen for $\mathrm{Cu}^{63}$ and $\mathrm{Cu}^{6 s}$ in the wire of the coil and for $\mathbf{A 2 7}$ in the aluminum sample itself. These NMR signals alloyed calibration checks for the magnetic field readings indicated on the "Pieldial" magnet pover supply. The design of the dewar system allowed the use of temperatures belor $4.2 \mathrm{~K}$. By pumping on the liquid helium the temperature could be lowered from $4.2 \mathrm{~K}$ to $1.9 \mathrm{~K}$. No signal enhancement was noted when the system was operated at $1.9 \mathrm{~K}$, but the signal to noise ratio could sometimes be improved this way due to reduced vibration because no bubbles were moving past the sample when the temperature of the helium was belor the lamda point.

The detection theory for the f m detection of RFSE signals has been discussed in detail by cleveland (51).

\section{B. Sample Holder Assembly}

A series of sample holder and coil assemblies were made with the idea of making them reusable for different samples. The construction of these assemblies will be discussed and then the technigue used to install and remove samples from the coil will be mentioned.

The sample holder assembly was constructed so it could be attached to the bottom end of a coaxial "cable" made of 
two sizes of non-magnetic stainless steel tubing, assembled one inside the other. This coaxial "cable" was used to support the sample holder between the pole faces of the magnet to be used. Each sample holder was made from a piece of Synthane rod which as turned down so the two ends of the holder had different diameters. The diameter of the small end allowed a slip fit into the large tube of the craxial conductor while the large end of the holder was made small enough to fit inside the narrow tail of the helium devar. The sample holder uas then removed from the lathe and mounted in a jig on an end mill. The jig was designed to guarantee that the axis of the diameters already turned would be perpendicular to the face being cut in this step. If the coaxial conductor holding the sample holder is vertical, then the bottom face on the holder will lie in a horizontal plane. Holes vere drilled in the narrow end and side of the sample. holder to allow the electrical connection for the coil to the center conductor in the supporting coaxial conductor.

The coil was preassembled and then attached to the sample holder. The dimensions of the desired coil were chosen based on the dimensions of the sample intended for insertion into the coil. A coil form made of steel shim stock or phosphor bronze was chosen that yould provide the proper width and thickness for the coil. The proper length was obtained by varying the number of turns adjed to the 
coil.

Once the coil form was prepared, the edges vere sanded lightly to remove all burrs that might increase the difficulty encountered when the coil is remored. The coil form was was then gripped by one end so the form was perpendicular to the jaws of the vise. This helped ensure that the cesulting coil would be rectangular rather than occurring at some skewed angle. The coil form was then coated lightly vith vacuum grease. The coil was then wound on the form using the desired gauge of magnet wire with a heary Polythermaleze insulation. After every two or three turns were added they were pressed snugly toward the jaws of the vise against the preceding turns. This resulted in an even, close-packed coil. After the desired length was reached, the vires were taped to the vise to keep the coil frod unraveling and then a smooth-jawed pair of pliers was used to squeeze the large flat sides of the coil against the coil form. This tightened the bends in the wire at the edges of the coil foral and resulted in a mach flatter coil. One side of the coịl was then thoroughig cleaned to remove all vacuum grease and GE 7031 varnish uas applied in very light coats. Care was taken to aroid the build up of a thick layer of varnish on this surface. Following a suitable time to allow the varnish to harden, the coil form was pulled loose from the coil to be sure the varnish had not penetrated the coil and vacuua 
grease enough to allow the coil to be cementel to the coil form.

The coil vas then ready to attach to the sample holder. A table vise was used to press the coil flat against the face of the sample holder until the adhesive dried but special precautions were taken to ensure that the plane of the coil would be perpendicular to the axis of the sample holder. The sample holder was mounted in a machined jig that held the sample holder axis perpendicular to the jaw faces of the vise. Solvents were now used to clean the second side of the coil. Several drops of GE 7031 varnish were placed on the flat face of the sample holder and the clean side of the coil was placed face down in the varnish. The assembly was then placed in the vise and pressure was applied to hold the coil flat against the sample holder face until the rarnish dried. It was found that the drying time of the varnish should not be decreased by heating because this caused undesired bubbles in the varnish.

After the varnish dried, the assembly was removed from the vise and all excess vacuum grease was removed. Then new coats of GE 7031 varnish vere added to strengthen the bond holding the coil on the sample holder and to add rigidity to the coil itself. The coil form was removed from the coil and the coil form assembly was ready for the insertion of a sample. 
It was desirable to be able to insert and remove a sample from a coil without damaging the sample. This led to the use of a thin (0.0004") mylar envelope to transport the sample into and ont of the coil. The mylar was cut in a long strip vith a width just smaller than the coil width. The strip was then folded with the closed side perpendicular to the long dimension of the strip. The open end of the foldel strip was inserted through the coil. Vacuua grease was applied to both inside surfaces of the folded strip near the fold. The sample was then placed inside the folded end. The strip and the sample were then drawn insile the coil. The vacuum grease was used to prevent any looseness in the coil and to hold the sample firmly in the coil. Very severe noise problems in the data were noted when the sample was able to vibrate loosely in the coil.

By using the thin mylar and the sample in the coil, the sanple thickness used in a coil had to be less than the thickness of the coil forn used. When choosing a coil to use with a sample, the coil form was selected where its thickness was roughly the same as that for tro layers of mylar plus the sample. Since the signal strength depends on the fraction of the coil volume that the sample occupies (51), a close fit for the sample-envelope combination in the coil is verp desirable. 
The sample could be removed from the coil by simply pulling the leading edge of the mylar envelope from the coil. Using this technique, sample Al12 was inserted and removed from coils four times and good signals were still found in each subsequent investigation.

\section{Oscillator Problems}

In the RPSE the rf oscillator serves two functions. It provides a probe sensitive to surface impedance changes in the sample which can be related to RFSE resonances. Also the frequency of the oscillator determines the skin depth in the metal for the rf fields and hence the width of the RFSE resonance $(\Delta H)$ as discussed earlier.

cleveland (51) has shown that for variable frequency oscillators and the detection method employed in this investigation, the signal represented by. the vertical axis on the RFSE data traces is given by

$$
(\mathrm{df} / \mathrm{dH})=\left[\left(\mathrm{n}^{2} \mathrm{~A}_{\mathrm{s}}\right) /(4 \pi \mathrm{L})\right]\left[\mathrm{dX_{xx }} / \mathrm{dH}-(2 \mathrm{c} / \omega \mathrm{L}) \mathrm{dR_{xx } / d \mathrm { B } ]}\right.
$$

where f=oscillat or frequency, $\omega=2 \pi f, n=t u r n s / l e n g t h$ of coil, $A_{S}=$ total area of the sample, L=inductance of the empty coil, $r=e f f e c t i v e$ total resistance of the coil=sum of coil resistance plus effect of sample surface resistance, and $x_{x x}$ and $R_{x x}$ are the imaginary and real parts of the sample's surface impedance. Since $(c / \omega L)^{-1}=Q=q u a l i t y$ factor of the coil-sample combination, it is seen that for large values of $Q$ the value of $d f / d H$ is proportional to $\left(d x_{x x} / d A\right)$. Equation 
24 is valid on 1 y for the case where $L$, $I$, and the capacitance of the tank circuit, $c$, have no frequency dependence.

Consider the results expected when taking data with two oscillators operating at the same frequency. The parameters $n$. As. I, and $r$ are the same for both oscillators since these are all determined by the coil-sample combination and not the oscillator circuit. The remaining parameters, $d_{x x} / d H$ and $d R_{x x} / d H$, depend only on the sample and the value of the magnetic field so the traces for the two oscillators should have the same shape. But this was not observed in all cases.

Angular data and frequency study data were taken for samples $A 12$ and Al 16. Examination of the data traces and the resulting frequency study plots indicated the presence of an unexpected problem. The shape of the background curves differed in some cases and corresponding RFS signal featuras were found at different field values for different oscillators. Figure 36 shows traces for oscillators \#0, \#1, and 2 taken at nearly the sane frequency. The presence on each trace of the proton NMR signal due to the GE 7031 varnish on the coil bears testimony to the closeness of the frequencies for each oscillator. Also the NuR signals on each are the same shape although some of the other features seem to be inverted. Notice the differences in the traces from oscillators 0 and 1 and the trace from oscillator $\$ 2$. Figures 37 and 38 show comparisons of oscillators \#2, and \#3 


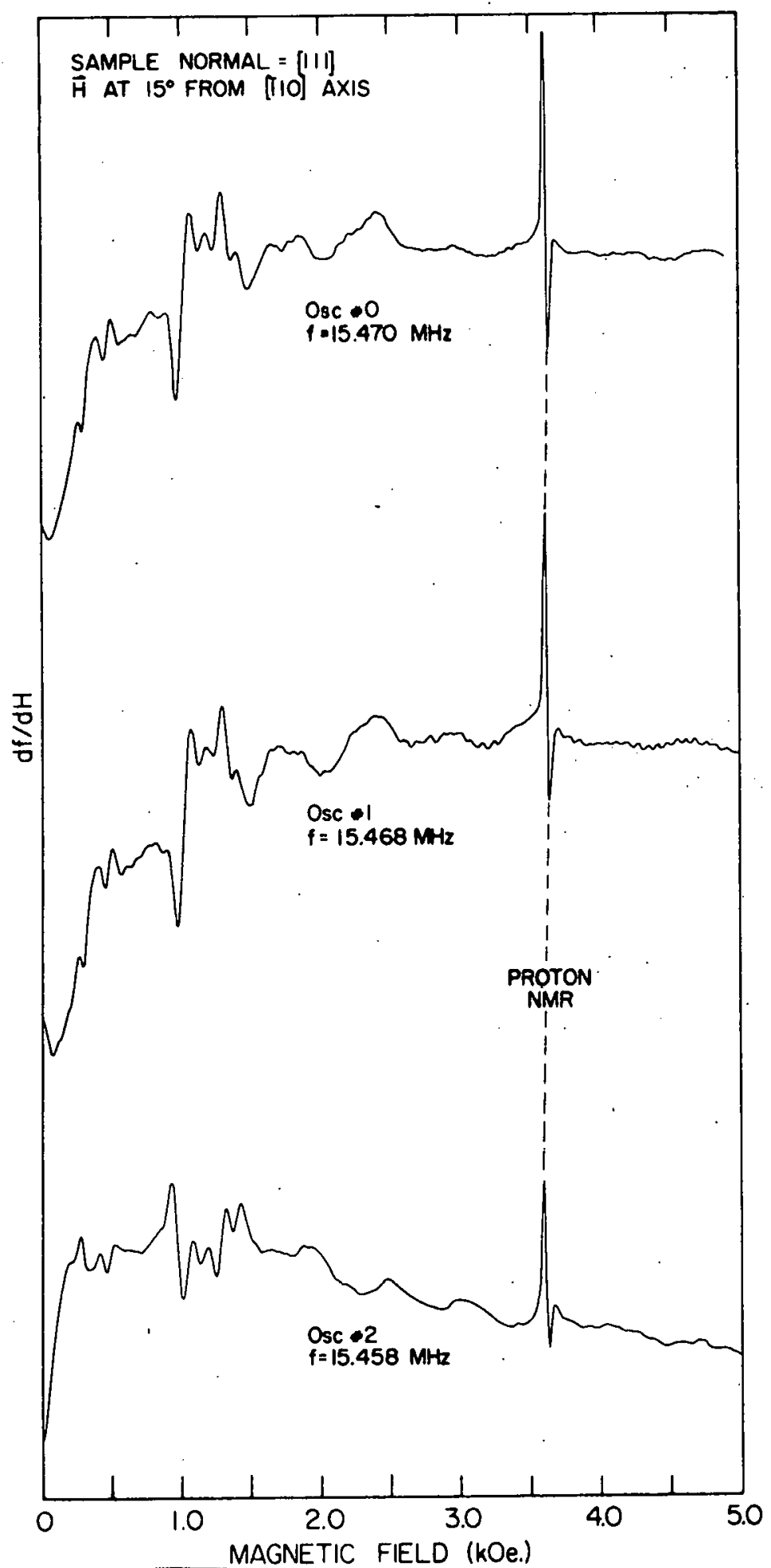

Pigure 36. Comparison of RP SE data traces taken with oscillators $\$ 0$; $\# 1$, and $\# 2$. Experiuental conditions were idontical for all traces 


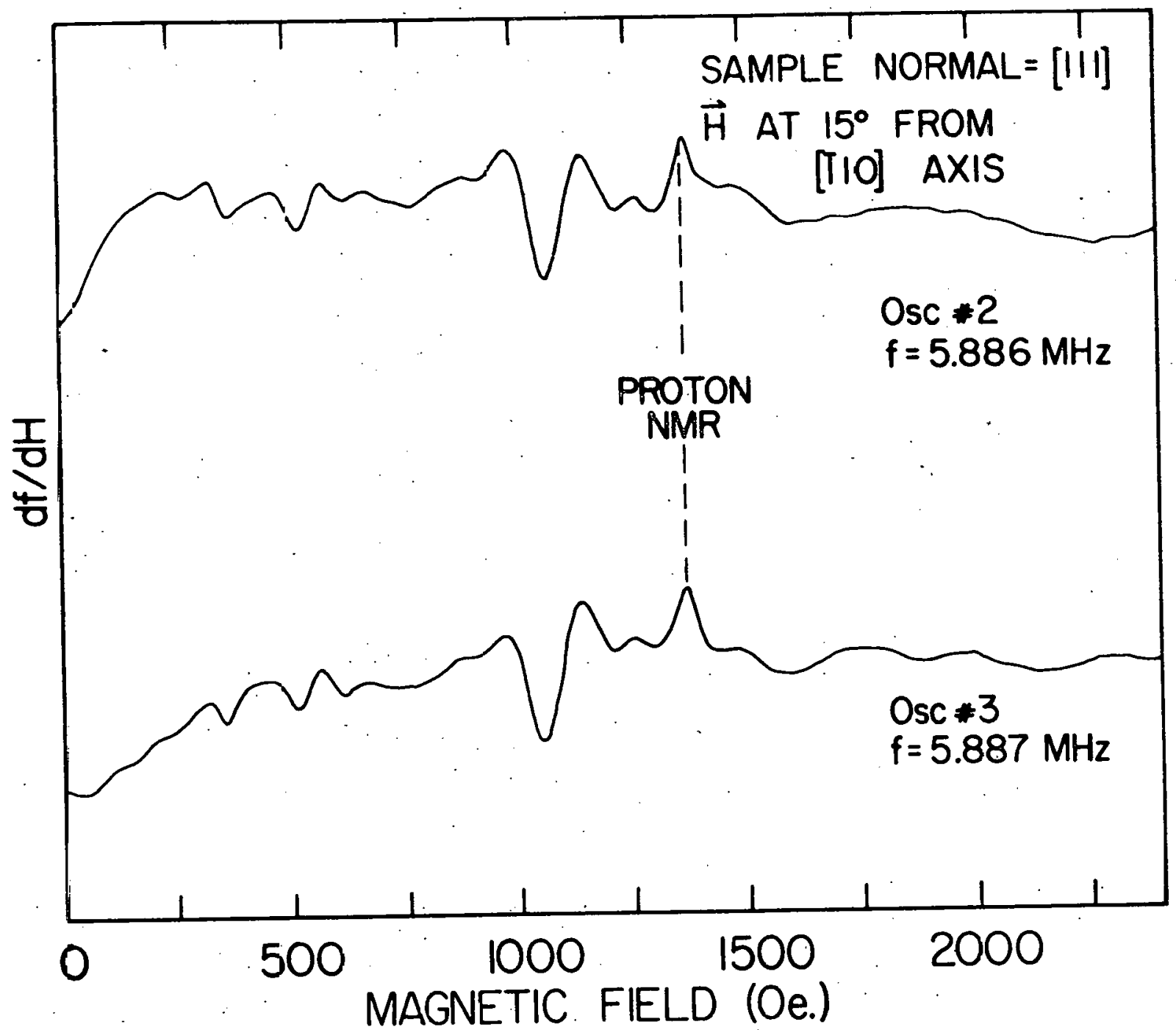

$\vec{\circ}$

Pigure 37. Sample traces for oscillators 2 an 13 for ideatical experimental conditions 


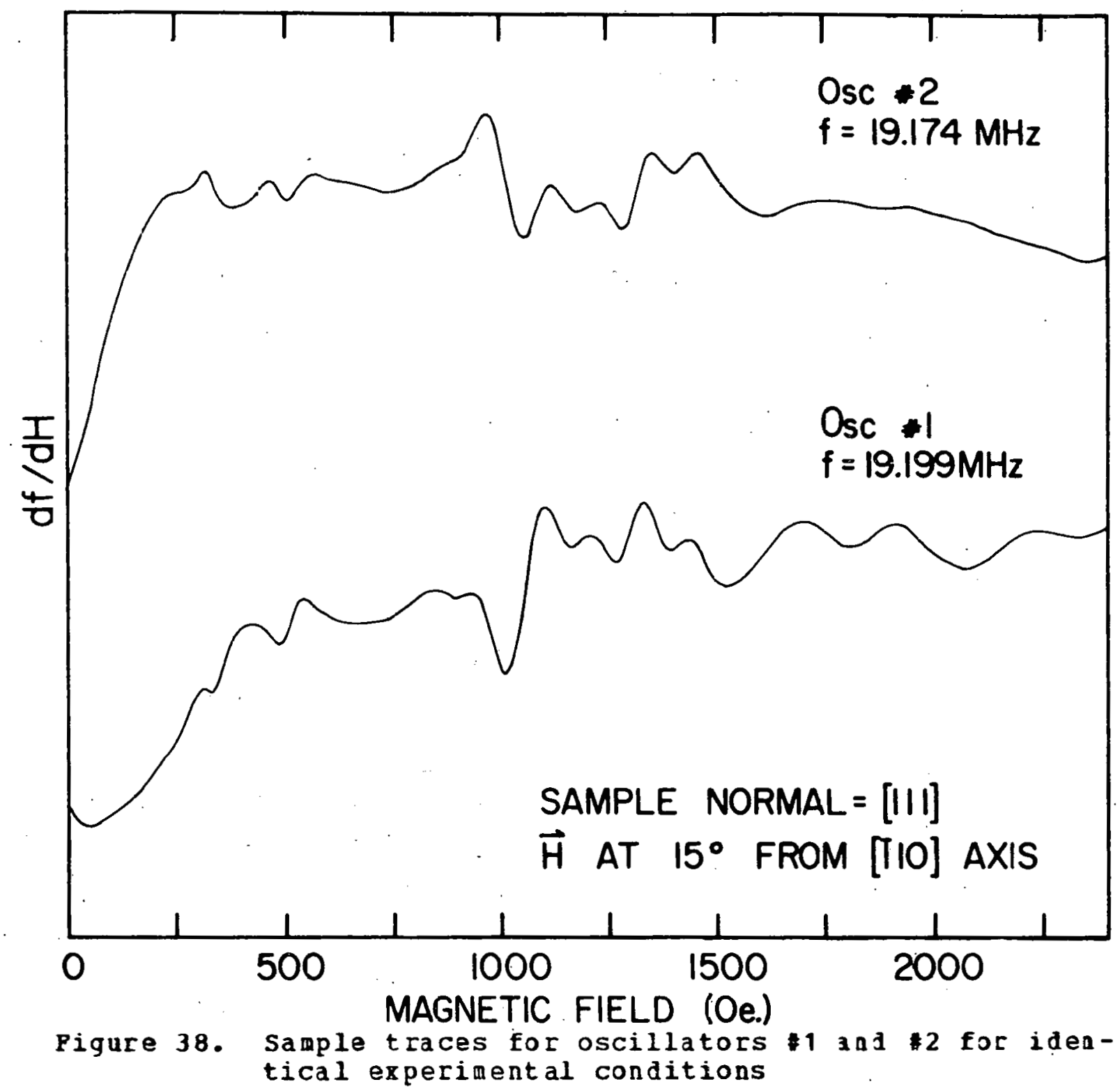


and oscillators 1 and 2 for a smaller range of magnetic field. Again note the differences in the traces from oscillators 1 and 2 . The traces from oscillators \#2 and \#3 show great similarity.

The problem is also evident if data for a frequency study are recorded over a frequency range requiring the use of all four oscillators. The result of such a study made on sample Al 16 as shoun in figure 39 vhere points resulting from oscillators 0 and \#1 are represented by the sybol " $x$ " and points from oscillators $\$ 2$ and $\# 3$ are represented by the symbol "+". It is seen that the sets of points, $+"$, form reasonably straight lines but they do not line up with the points from oscillators 0 and 1 . This is extremely evident in the cegion of frequency overlap for the two sets of data. One explanation considered was the possibility that the high frequency data points were actually at higher values of frequency than the Monsanto counter indicated, perhaps two or three times, the measured values. If this were true, the shift to lower field values would result from the natural narroving of the skin depth uith increasing frequency, the very effect that leads to the frequency study concept. The waveforms of the signals leaving the oscillators vere examIned with a vide band oseilloscope and found to be compatible with the frequency counter readings. The signals were not pure sine waves but it was not possible to see if the distor- 


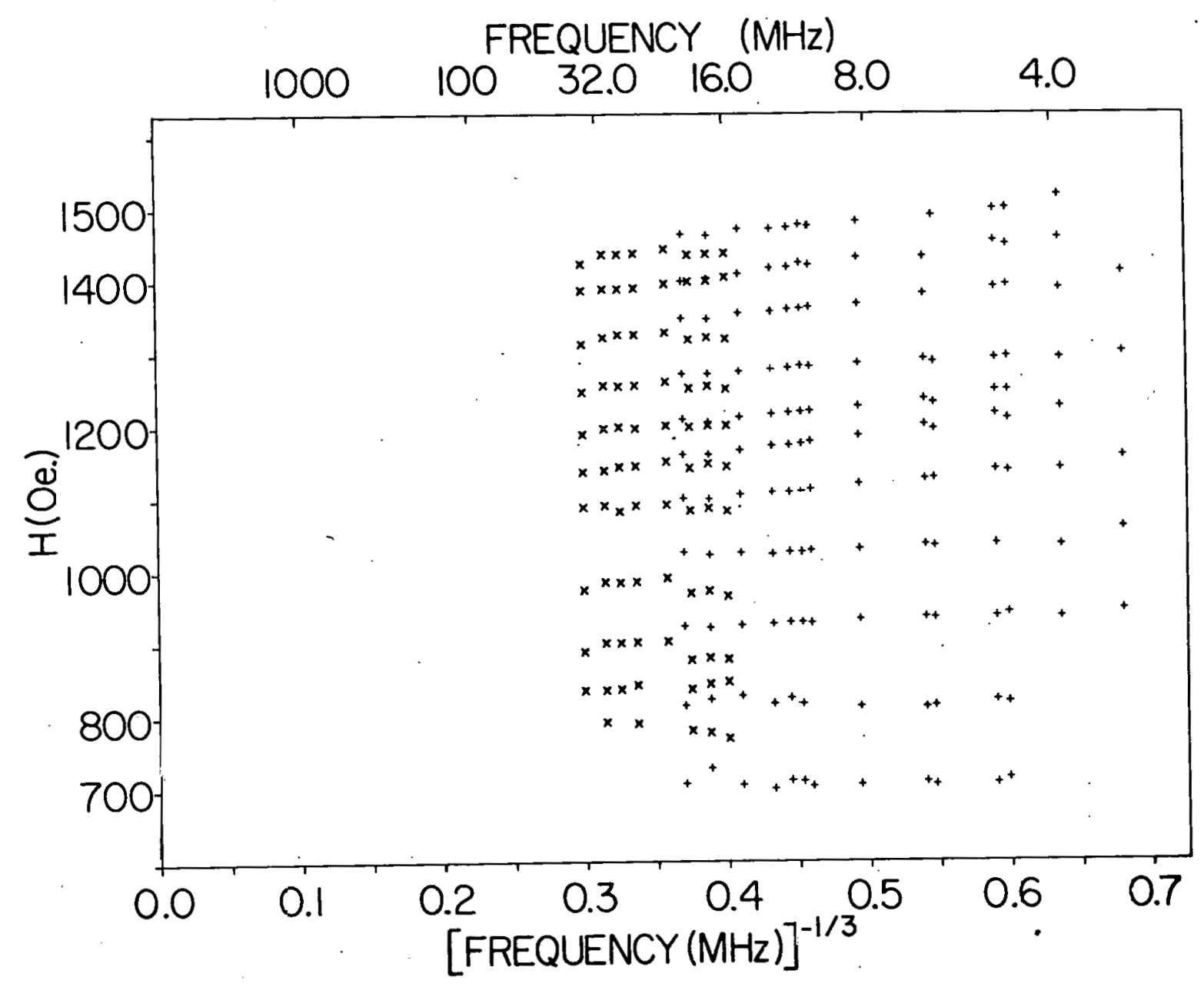

Figure 39. Frequency study plot for Al 16 with data from oscillators $\# 0$ and $\# 1$ ("x") and 2 and $3(n+n)$ 
tion was actually present on the coil or if it was introduced in the follower circuit mounted on the oscillator circuit board. The magnetic field values for the proton MMr signals also agreed with the frequency readings.

Variations in the magnitude of the rf fields at the sample vere considered. Additional resistance was added to the coaxial line between the oscillator and the coil to decrease the rf currents flowing in the coil. Data traces vere taken and compared as each increment of resistance vas added. No effect yas seen on RFSE signal locations or the background signals. The signal to noise ratio decreased as resistance was aded until the oscillations finally ceased. Ading resistance to the line would increase the value of I in Equation 24 and affect the value of df/da if the second tern in that equation was noticeable for these experimental conditions. No such effects were noted.

The clipper circuit was examined to determine if it could be leading to the problem in some unexpected manner. Signals were recorded using four other clipper designs with no change observed in signal locations. again the only effect was on the signal to noise ratio. The signal was also recorded without a clipper and the same signals were found again. This was true eren wen the receiver was tuned to the tenth harmonic of the oscillator's fundamental frequency. This indicated. either a non-linearity in the signal process- 
ing by the follower on the oscillator board itself or the presence of harmonics in the actual oscillator circuit. The search for harmonics in the oscillator circuit was directed at observing the proton NMR resonance for harmonics of the oscillator's fundamental frequency if these harmonics did in fact reach the coil.

The first evidence of actual harmonic content at the coil was observed for tube oscillator A were a proton NMR signal was observed at a magnetic field corresponding to three times the fundamental frequency of the:oscillator. This oscillator had been used in an earlier RFSE investigation (51. Cleveland oscillator \#3) and gave RFSE signal traces in this investigation that were the same as those for oscillator \#1. In Pigure 40 the upper trace was taken with oscillator A for the magnetic field at an angle which yielded good RPSE signals. The lover trace was taken for a magnetic field direction that maximized the MM signals and minimized the RFSE signals. The proton NMR signal is seea in response to the fundamental ( $\left.f_{0}\right)$ and its second harmonic $\left(3 f_{0}\right)$. From Figure 40 it is seen that the df/dH signal due to the fundamental MMR signal is roughly seven times as strong as that due to the second harmonic.

Similar examinations vere carried out to evaluate the harmonic content in the signals obtained asing transistor oscillators \#1, 2 , and 3 . It vas impossible to examine 


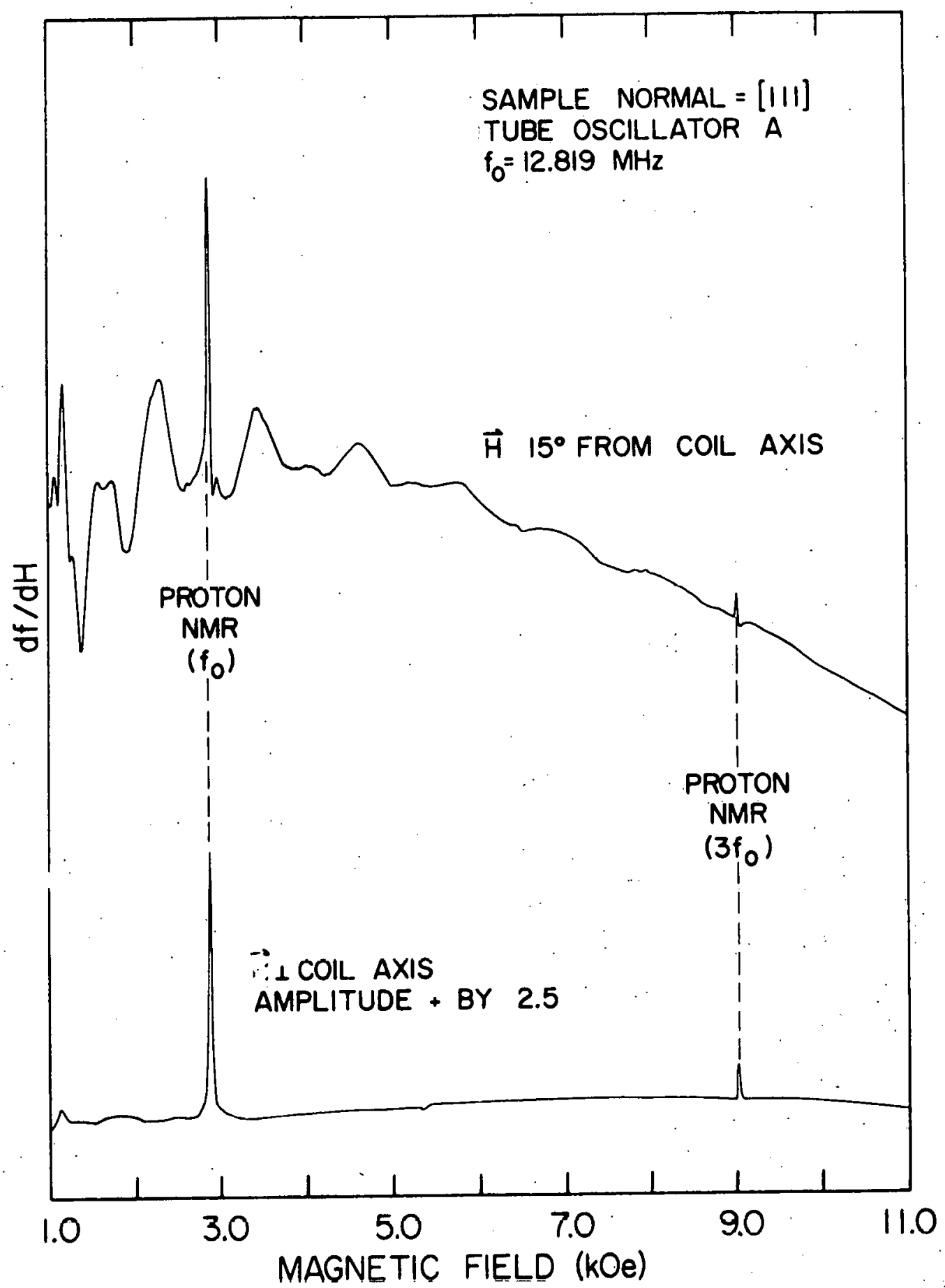

Pigure 40. Sanple data traces illustrating the presence of the second harmonic found in the signal from. tube oscillator a 
oscillator \#0 due to the limited range of magnetic field supplied by the electromagnet. The results of this survey vill be sumarized below.

Oscillator \#3 showed no indication of the second harmonic NMR signal. NaR signals vere seen due to Cu63, Cu6s, and 4127. The amplitudes of these signals as measured directly from the data sheets were about $7 \%, 3 \%$, and $8 \%$ of the strength of the fundamental proton NMR signal.

Data traces obtained using oscillator \#2 indicated a. proton NAR signal due to the second harmonic of the measured frequency. This signal had an amplitude of about $2 \%$ of the fundamental proton signal. The MHR signals due to $\approx u^{6} 3$ and A127 were also seen with this oscillator and were a little stronger than the second harmonic peak.

oscillator \#1 was examined and no harmonic content vas noted. This investigation was limited by the field range of the electromagnet used.

A brief study was made with tube oscillator a operating between $13.1 \mathrm{Mz}$ and $15.3 \mathrm{MHz}$ and large changes in the harmonic content were noted. These changes were roughly an order of magnitude as measured on the data traces. Caution should be used when considering these values. The apparatus used in the $f m$ detection method is not designad to retain exact anplitude information for the signals, and amplitude fluctuations are often seen in traces taken under the same 
experimental conditions. Even so, the vide range of amplitude seen here can be considered to reflect a real trend in the signal's haraonic content.

Another attempt to evaluate the harmonic content of the signal on the sample holder coil utilized an external pick-up coil and an auxiliary rf signal generator. The pick-up coil vas placed in close proximity to the sample holder coil (just outside the glass dewarl and the coil output was fed to the communications receiver input. The signal strength as indicated by the receiver s-meter was recorded ith the receiver tuned to each harmonic value. The If generator was then tuned to the same frequencies and the signal level that gave the same signal strength vas recorded. This study indicated that oscillator 3 applied the purest sine wave to the sample holder coil. Tube oscillator a and transistor oscillator shoved the greatest amount of harmonic content on the sample holder coll. Oscillator \#2 exhibited less harmonic content than 1. Problems a rose in these measurements associated. with resonances within the probe coil, local radio station pick up, and impedance atching of the coil to the receiver. Following these studies oscillators $\$ 0$ and 1 were discarded and oscillators $\$ 2$ and $\$ 3$ were used to take angular data on the aluminum samples. The reasons for this decision are given belov.

1) The large harmonic components found in oscillators \#0 
and and the similarity of their data traces to those obtained using oscillator \#1.

2) The inconsistencies in the high frequency data points on Figure 39 The points taken with \#0 and \#1 do not show the standard straight line form expected.

3) The very small harmonic content found in oscillator \#3 and the similarity between its traces and those for $\# 2$.

4) The data for 2 and $* 3$ show good alignment on the frequency study shown Figure 39 :

Prom the data recorded, it is seen that the $f_{0}$ and $3 f_{0}$ signals do not behave as two independent signals affecting the coil-sample system in a linear manner. clipping a signal of frequency $f_{0}$ generates harmonics at $2 \mathrm{f}_{0} .3 \mathrm{f}_{0} .4 \mathrm{f}_{0}$. etc. clipping for a signal at $3 f_{0}$ results in harmonics at $6 f_{0}$ 。 9fo. 12fo. etc. For a linear superposition of these signals, both signals will be present for verp few frequencies that could be tuned on the communications receiver (e.g. $6 f_{0}$. 9fo. etc.l. But experimentally it was found that both NMR signals could be observed when the receiver was tuned to adjacent harmonics of $f_{0}$. This indicates that for the interactions taking place in this experiment, the idea of a linear superposition of responses to the signal on the coil is not ralid. 


\section{Sample preparation}

All samples prepared vere taken from a rectangular parallelepiped (16 m $\times 17 \mathrm{~mm} \times 65 \mathrm{~mm}$ ) of $99.9999 \%$ pure aluminum obtained from cominco merican. Inc. of spokane, Mashington. The residual resistance ratio (the resistance at $300 \mathrm{~K}$ divided by the resistance at $4.2 \mathrm{~K})$ quoted by the manufacturer was 13,000. (After spark cutting had been completed a thin needle of aluminum was examined using a standard four probe technique and the resistance ratio vas found to be greater than 8500. Greater accuracy could not be obtained due to small magnitude of the signals being measured and the noise level in the measuring systen.). The ingot was not a single crystal but after chemical etching in a mixture of phosphoric, sulfuric, and nitric acids (148), large crystallites vere visible to the naked eye. The last $20 \mathrm{~mm}$ of material on one end of the ingot appeared to be a single crystal. That end of the ingot was then examined using laue $x$-ray backscattering techniques. This was done to locate the three cubic axes in the crystal so cuttings could be planned to minimize crystal wastes when $[100],[110]$; and [111] normal samples vere to be prepared.

For each orientation of samples to be cut the crystal was aligned to within $1^{0}$ of the desired axis using the $x$-ray techniques. Several thin plates (1 min thickness) were then cut off the end of the ingot with a moving-vire spark cutter. 
Extra plates were cut in each case to allow the preparation of five or six RFSE samples for that orientation before further cutting from the ingot would be required. Each slice was then re-etched in the acid solution to allow a visual check for any crystal boundaries in the face of the slice. Each slice was then spark cut into rectangular plates with surface dimensions of roughly $8 \mathrm{~mm} \times 9 \mathrm{~mm}$. These rectangular plates vere then prepared as individual samples. For further preparation of the sample's first side, the sample was attached to an adjustable lapping plug as discussed by cleveland (51) and $x$-ray techniques were used to align the crystal to within less than $0.5^{\circ}$ of the desired axis.

Por use in the RFS, the samples need to have very flat, parallel faces and be as free of damage as possible. Therefore a method of sample preparation would be preferred if it yould not induce large strains in the crystal. For this reason, the technique of spong and kip (31) was tried. This involved a nodified electropolishing process which incorporated a lapping motion against a Teflon cloth subaerged in the acid solution. The sample was mounted to an aluminum lapping jig using a standard mixture of graphite and Duco cement. The portion of the jig surrounding the sample was their coated with a lacquer to linit current flov to the sample surface. The lapping jig acted as the anode for the electropolishing and the cathode was a sheet of stainless 
steel mounted below the reflon lapping cloth. Very flat surfaces resulted from this technique, but the technique was discarded wen it was found that the sample's second surface could not be prepared without severe danage occurring on the first side that had already been prepared. This resulted because the acid solution could not be prevented from seeping behind the crystal and re-etching the first side. This problew was compounded by the smooth surface on the first side and the fact that the Duco cement and graphite did not adpere vell to the finished surface. Different mixtures of the conducting adhesive were tried as vell as a silver paint but none were successful for holding the sample down and preventing the acid from etching the first side.

Good samples were then prepared using mechanical lapping techniques. Samples vere attached to the adjustable lapping plug using beeswax. Lapping was done in four steps. The first employed \#600 grade grit paper and methanol as a slurry. This was used until all signs of roughness due to spark cutting were removed and the sample thickness vas reduced by about one third. Next a $4 / 0$ emery paper was used to remove all signs of scratches left by the 600 grade grit. Then one micron alumina abrasive was used with a water slurry for lapping on a Teflon cloth. This was continued until the surface had a uniform texture when viewed under a low power microscope. The surface at this point was not 
shing but rather had a smoky appearance. The final lapping was done using a comercial red rouge with methanol on a Teflon cloth. The size of the abrasive particles in the rouge were less than $1 / 3$ of a micron in diameter. Pollowing this the surface appeared very flat and shiny to the naked eye. Some small scratches could be seen under the microscope but the surface was generally very nice. Care was taken to keep the alumina abrasive from being transferred to the Teflon used for the final lapping.

The sample was removed from the lapping plug and the plug face itself was then lapped flat to ensure that it was perpendicular to the axis of the plug. The sample vas then reattached to the plug so the unfinished surface could be prepared. Before any work was done on the surface the crystal was $x$-rayed again to check alignment. This was done to guard against the chance of any foreign particles in the adhesive getting under the crystal and preventing it from being flatly held against the plug face.

The second face was then prepared following the same steps used for the first side. The only difference being that the $\$ 600$ grade grit paper was used until the desired sample thickness was approached. Thickness was nonitored during preparation using a Leitz optometer.

The sample was then thoroughly cleaned vith warm solvents (petroleum ether, acetone, and trichloroethane) to 
ensure a uniformly clean surface for dip electropolishing. A standard electropolishing solution of $6 \%$ perchloric acid in methanol was used in a dry ice and acetone bath. The current density was set in compliance with the guide lines set forth by Metz (149). The experimental findings of Metz make it possible to monitor current and voltage changes to avoid current densities that will probably lead to pitting rather than a smooth shiny surface.

Following the electropolishing, the surfaces vere shiny and flat but showed signs of rounding near the edges. In some samples a few scratches were visible under the microscope but the majority of the surface was clear of such obvious defects. Further x-ray photographs taken at this time shoved sharp distinct spots with no signs of twinning or obvious crystal damage resulting from the surface preparation. E. Sample. Thickness

The thickness of the samples has been determined by two methods. The first involves measuring the mass of the sample using an accurate analytical balance. The calibrated dimensions of the sample are then measured using a calibrated traveling microscope. The linear dimensions of the surface are then used to calculate the area of the sample. The average thickness was then calculated by using the known density for aluminum $\left(2.702 \mathrm{gm} / \mathrm{cm}^{3}\right)$. These values are shown in the third colum of Table 2. This procedure includes any 
rounding near the edges into the average. Thus this technique may yield an average that is lower than the average away from the edges if rounding problems are important for a specific sample.

Table 2. Average thickness for aluminum samples

\begin{tabular}{|c|c|c|c|c|}
\hline Sample & $\begin{array}{l}\text { Normal } \\
\text { axis }\end{array}$ & $\begin{array}{l}\text { Average } \\
\text { thickness }\end{array}$ & $\begin{array}{l}\text { Average } \\
\text { thickness }\end{array}$ & $\begin{array}{l}\text { Microscope } \\
\text { readings }\end{array}$ \\
\hline & & $\begin{array}{l}\text { (by mass) } \\
\text { (microns) }\end{array}$ & $\begin{array}{c}\text { (by microscope) } \\
\text { (microns) }\end{array}$ & \\
\hline
\end{tabular}

$\begin{array}{llllll}A 1 & 12 & {[100]} & 172.3 & 172.3 \pm 4.9 & 36 \\ \text { A1 } 13 & {[100]} & 150.0 & 149.7 \pm 10.6 & 39 \\ \text { A1 } & {[110]} & 180.9 & 188.4 \pm 7.8 & 36 \\ \text { A1 } 16 & {[111]} & 112.5 & 110.8 \pm 7.8 & 48\end{array}$

The second method involves taking a series of actual thickness measurements across the face of the sample using the focal point of a microscope with a calibrated lens movement. The sample is first placed on a flat metal plate and held in place with beeswax. The sample vas then measured using a microscope with a calibrated table motion in two directions perpendicular to the axis of the lens system. This made it convenient to take data at equally spaced points across the surface. Thickness measurements vere recorded at 
the points on a square grid where the grid lines were separated by 0.050 inches in both directions. The recorded values were averaged and the standard deviation was calculated for each sample. Pigure 41 contains detailed information about the distribution of the thickness measurenents for the four samples of importance in this experiment.

The arerage thicknesses and standard deviations for the samples are listed in column four of Table 2. The number of readings taken for each sample is recorded in the last column of the same table.

It should be noted that excellent agreement in the average thickness resulting from the two techniques is found for samples A1 12, Al 13, and Al 16. The result for al 7 shovs agreement within the indicated standard deviation. The disagreement is also compatible yth effect of severe rounding near the edges of the sample.

None of the samples are as flat as desired. On the type of graph seen in figure 41, a flat sample vould find all reading of the thickness at the same point. A redge-shaped sample would lead to a graph with an even distribution of values from the minimum thickness to the maximum thickness. 

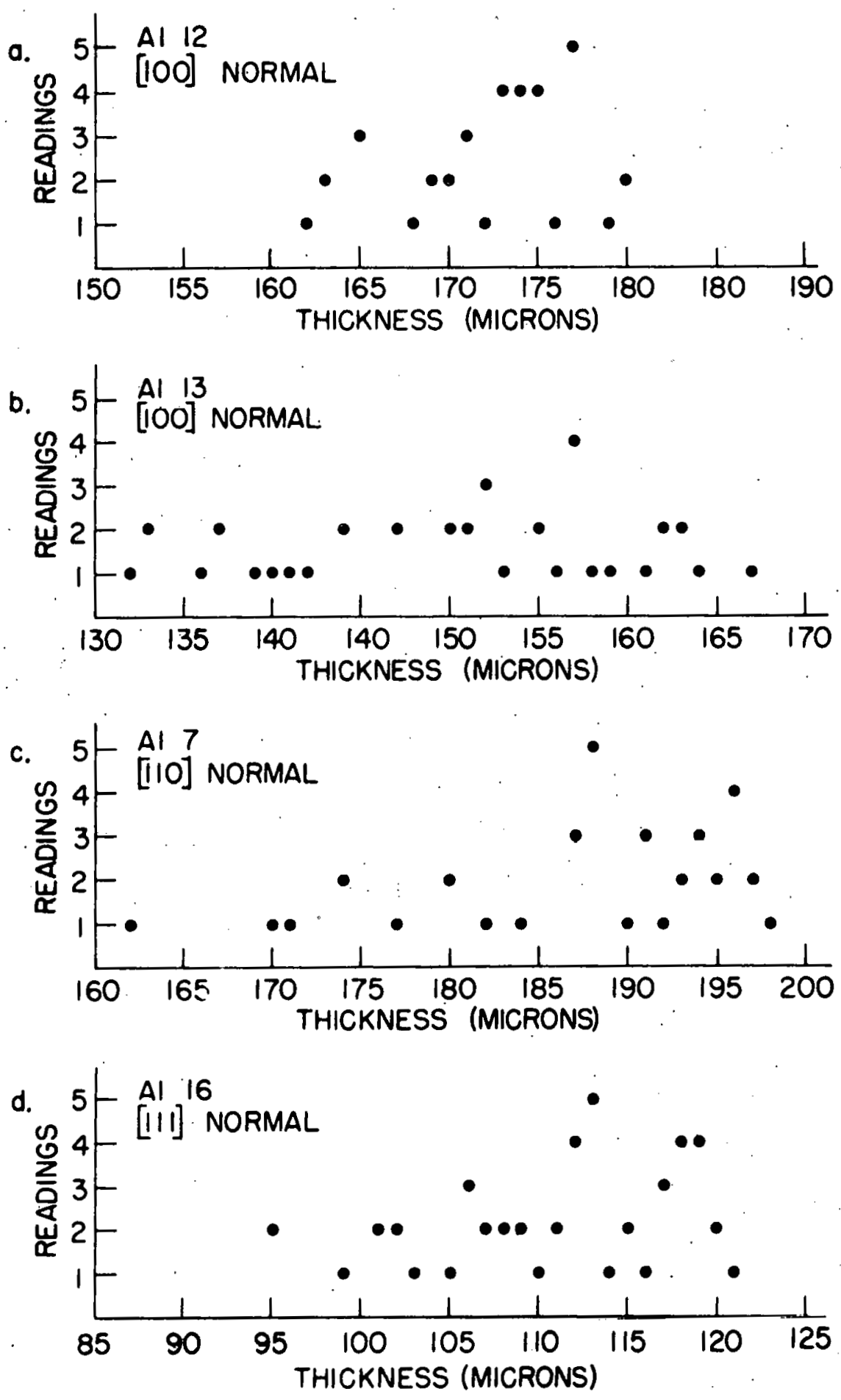

Pigure 41. Distribution of thickness measurements for samples al 12, A 13, Al 7 , and Al 16 
VI. DATA AND DISCOSSION

During the course of this experiment angular and frequency study data were recorded for a series of samples with [100], [ 110$]$, and [111] axes normal to their respective sample surfaces. The folloving discussion will deal with the raw angular data, the frequency study data, and the resulting angular dependence of the calipers and the electron orbits contributing to the individual signals in that order.

\section{A. Rav Angular Data}

The angular data considered were taken using samples Al 12 (a [100] normal axis), Al 7 (a [110] normal axis), and Al 16 (a [111] normal axis). The symmetry of the fS then requires that the RPSE signals show $900,60^{\circ}$, and $180^{\circ}$ rotational symetry for the respective samples. This can easily be seen by examining the FS cross section overlay figures for the normal directions (Figures 19, 20, and 21).

The Al 12 ([100] axis) data and Al 16 ([111] axis) datz did not demonstrate the proper angular symetry. First consider the data obtained using al 12 .

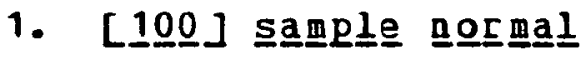

The initial data vere recorded with one of the cubic crystal axes parallel to the coil axis. A rectangular plot of the uncorfected field values versus the magnetic field angle is shown in Figure 42. Data are shown for every feature on the data traces and not simply the lowest feature of 


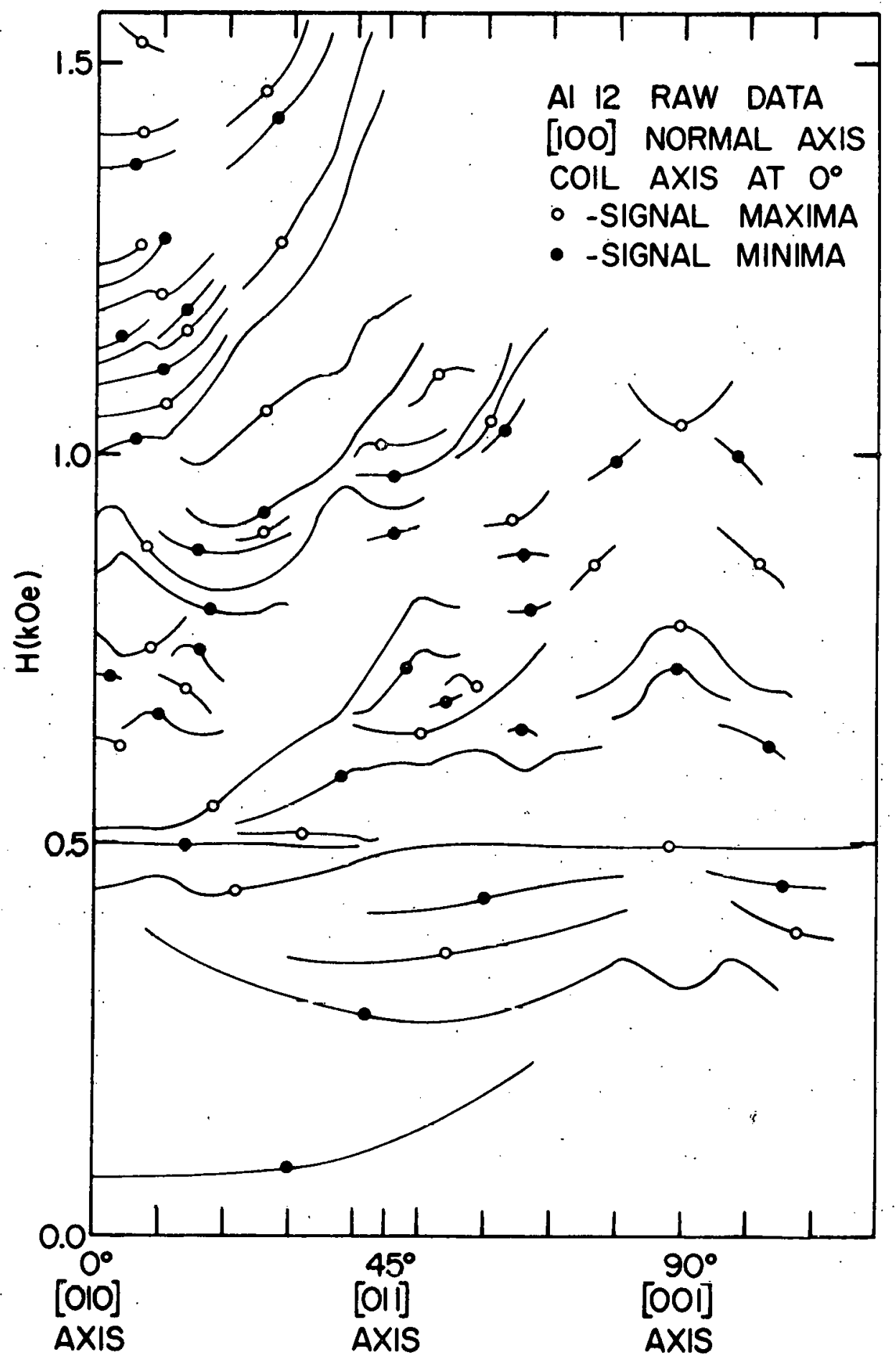

Figure 42. Angular dependence of raw angular data for [100] sample normal with the coil aris along a cubic crystal axis 
every signal. Data are not shown for negative field angles because they were simply a mirror image of the data shown.

From the contour orerlay plot (Figure 19) it is seen that the data should possess both $90^{\circ}$ rotational symmetry and mirror symmetry about the [ 010$]$ and [011] axes in the plane of the sample. The mirror image about the [010] axis coinciding with the coil axis did occur but there was no mirror imaging about the [ $\left.\begin{array}{lll}0 & 1 & 1\end{array}\right]$ axis or any 900 rotational symetry.

Some apparent deviation from the required symmetry could be expected due to the decrease of experimental sensitivity as the magnetic field approached $90^{\circ}$ on the figure, but this can not explain the deviations from mirror symmetry to be seen in the region of the [011] axis. In this small angular range the sensitirity should not change greatly. Examining the features in this region for field values near 600-700 0? and near 1000 oe shows that they reach their extrema about $51^{\circ}-52^{\circ}$ from the [010] axis and not at $45^{\circ}$ as expected.

The sample was removed from the coil and then replaced in the coil after a $90^{\circ}$ rotation. This placed the other cubic axis in the plane of the sample parallel to the coil axis. Angular data were recorded again and the features were unchanged from those seen in Figure 42.

The sample was again removed from the coil. A new coil vas prepared that allowed the sample to be placed inside the 
coil with a [011] axis parallel to the axis of the coil. Due to space limitations in the derar system, this coil did not completely enclose the sample as the previous coils had done. It simply belted the sample, passing across tro opposing corners of the rectangular sample. The coil width was adequate to cover more than one-half of the sample's area.

The resulting data are shown in Figure 43. Two striking features are to be noted. Pirst, many of the features seen earlier near 00 are noy seen at $45^{\circ}$ as expected. This is most obvious for features near 1000 oe. Second, some features still do not demonstrate the expected symmetry and these features are again symetric about the coil axis.

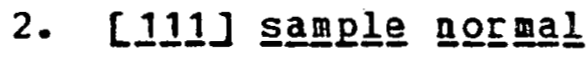

The symmetry problems for sample Al 16 (a [111] normal axis) are not as pronounced as those just examined. Much of the apparent problem here may result from the complexity of the signals seen near the [10T] ares. Figure 44 shows the data for Al 16 with the [10 1 ] axis $7^{\circ} \pm 1^{0}$ from the coil axis. To resolve the data near the [10 $\overline{1}$ ] axis, data yere taken at much closer intervals than for the remainder of the angular range. Fourteen angles vere examined in the range from 00 to $\pm 10^{\circ}$ and then $2^{\circ}$ intervals were used outside that region. The extreme care taken near the [10 1 ] axis vas required because the features on the traces were changing very rapidy as the angle was varied. To avoid misinterpretation of the 


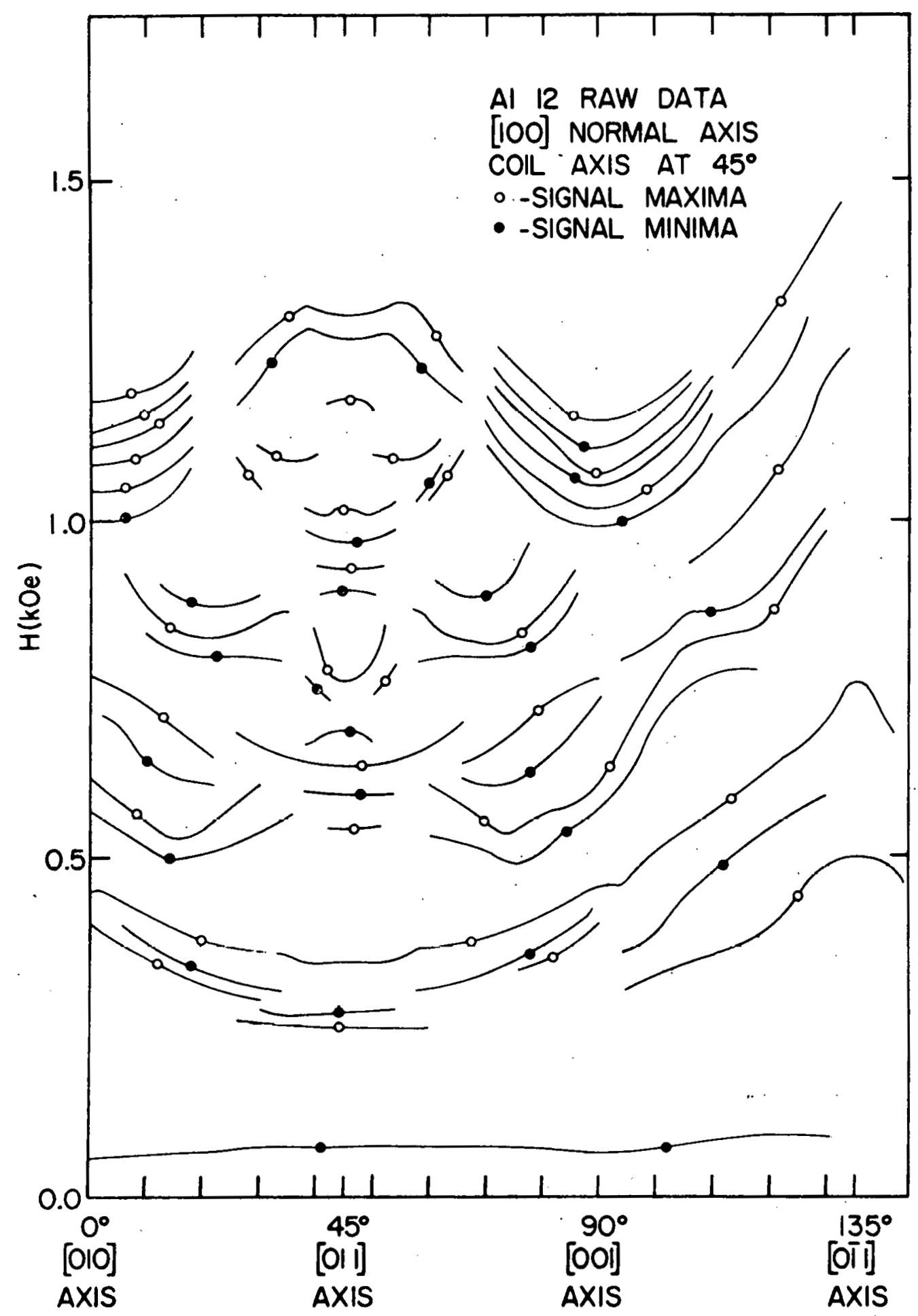

Figure 43. Angular dependence of raw angular data for [100] sample normal ith the coil axis at $45^{\circ}$ from $a$ cubic crystal axis. 


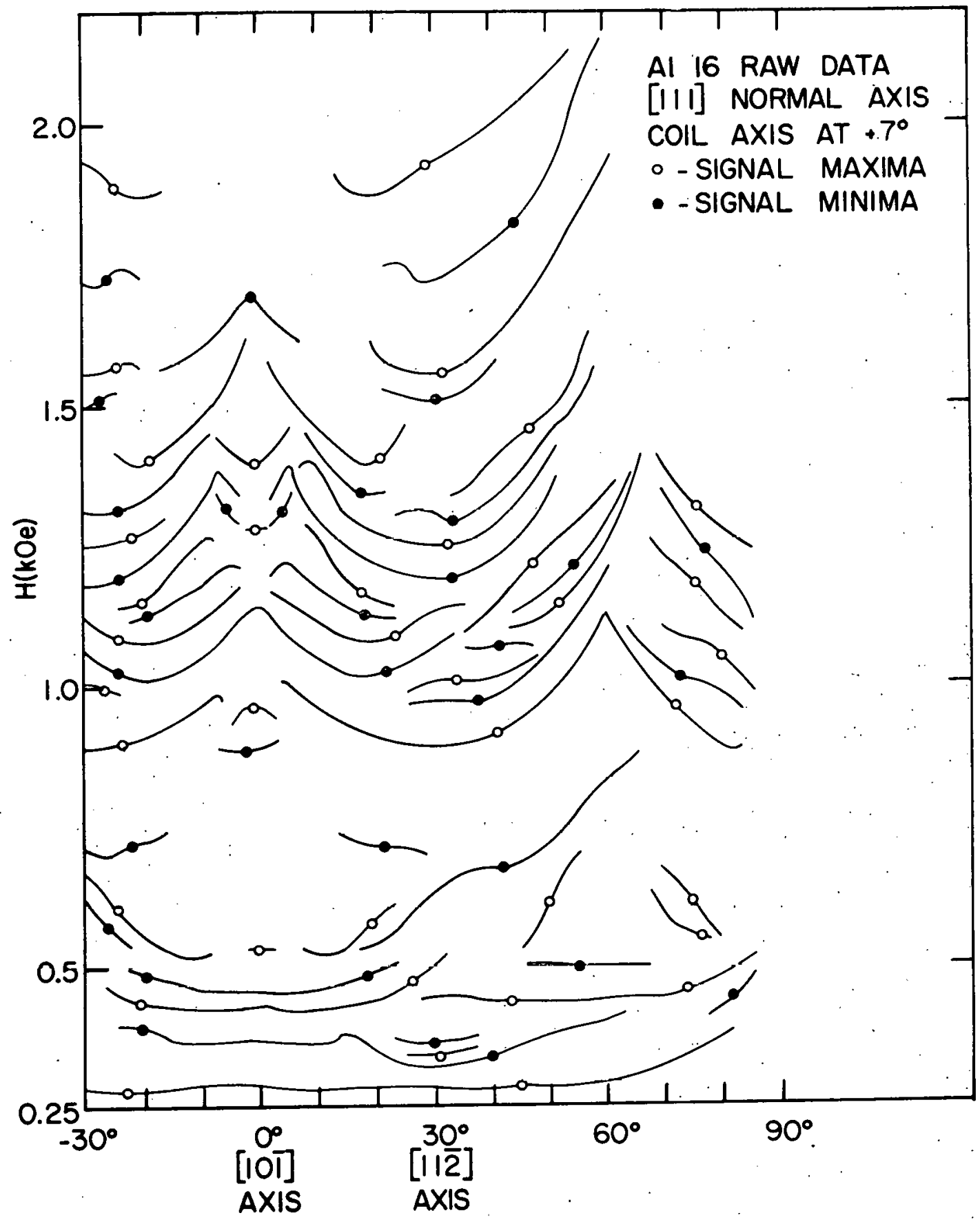

Figure 44. Angular dependence of raw angular data for [111] sample normal with the coil axis $7^{\circ}$ fron the [ 101 ] axis 
data, the angular intervals vere decreased to allow accurate determination of the behavior for individual features.

Some features near $60^{\circ}$ (the adjacent [ $\left.\overline{1} 10\right]$ axis)

continue rising to higher field values beyond the $\$ 600$ lines. This disagrees with the symmetry required. Interpretation of this region is complicated by the reakened signals due to the angle between the coil axis and the applied magnetic field and the complexity of the equivalent region seen near 00

Another symmetry problem exists near the [ $11 \overline{2}$ ] axis where mirror symmetry is required for the data. The small peak near $30^{\circ}$ and 1100 oe does not demonstrate mirror symmetry. This feature occurs at larger field values for 0 above $30^{\circ}$ than it does below $30^{\circ}$ for equal distances from the $30^{\circ}$ 1ine. This again is evidence of a symmetry problem in the data.

The sample was removed from the coil and reinserted after a $90^{\circ}$ rotation in an effort to obtain data complementing that seen in Figure 44. This is the same procedure that was followed for the [100] sample as previously discussed. A plot of the data obtained is shoun in Figure 45. Notice that the general features of these data do show symetry ander a $60^{\circ}$ rotation as required, but the feature near $0^{\circ}$ ( $[11 \overline{2}]$ axis again) and 1100 oe now does not show mircor symmetry about the 00 line. 


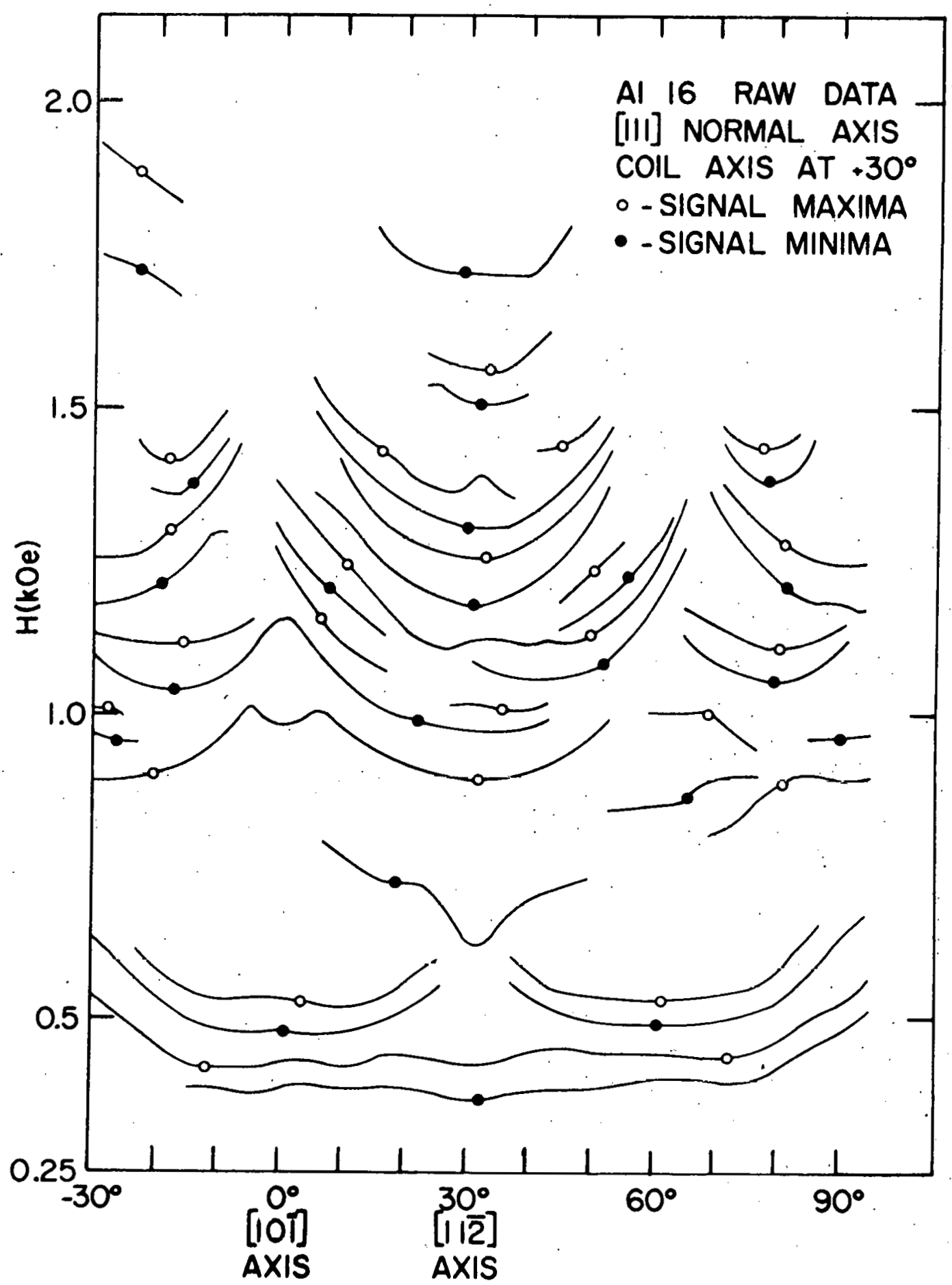

Pigure 45. Angular dependence of raw angular data for [111] sample normal with the coil axis $30^{\circ}$ from the [ 101 ] axis 


\section{3. [11ㅇ] sample 므으프믄}

The raw data from Al 7 (a [110] sample normal) followed the symmetry pattern expected. The data therefore presented no unusual problems and will not be discussed here. The re: sulting calipers will be discussed later in the text.

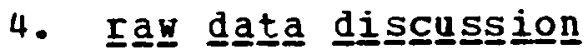

Prom the raw data presented above, the worst symmetry problems occurred for Figure 42 where the data for a [100] sample ith a cubic axis wlong the coil axis were presented. This figure showed unexpected symmetry for all features above 500 0e. The data for the same sample following a $45^{\circ}$ rotation inside the coil (a [011] axis along the $20 i l$ axis), (Figure 43) showed the proper symetry for all features above 1000 oe for angles that gave reasonable sensitivity for the RFSE signals (ie. $\pm 75^{\circ}$ from the coil axis). Below 1000 Oe some of the data does not follow the expected pattern at angles greater than $\pm 45^{\circ}$ from the coil axis.

The problems with the [111] data were considerably less. The complexity of the signals near the [10 $\overline{1}$ ] axes could easily result in misinterpretation of data traces for magnet angles where the RPSE sensitivity is diminished. Thus the only real question relates to the lack of mirror symmetry about the [ $11 \overline{2}$ ] axis for the feature near 1000 oe.

It should be noted here that for the data presented in Figure 44, the coil axis and the [10 $\overline{1}$ ] axis in the sample 
vere $70 \pm 10$ from each other. Thus if data depending only on the angle between the coil and the crystal axis occurred here, their symmetry axis should be different than the symmetry axis for the RPE data resulting from the PS. This is not seen in the Al 16 data.

Asymetry in the data could have been caused if the magnetic field was not applied parallel to the sample's surface. This could give rise to data with $180^{\circ}$ symuetry if the signals vere affected by the tilting. Several measurements vere made to examine this possibility.

The magnet assembly uas examined to determine if its axis of rotation was along a vertical axis. If the ragnet base plate had been installed poorly this problem could have arisen. The deviation from the vertical axis vas found to be 0.600 . The deviation was in a direction that would cause the effects of the tipping to be symmetric about magnetic field angles of $120^{\circ} \mathrm{R}$ or $150^{\circ} \mathrm{L}$ as seen on the magnet base plate. The problems of data symetry are not compatible with these findings as their symetry axes occurred far from either of these axes.

Data vere also taken with the top of the sample holder displaced horizontally from its normal position. This motion caused the stainless tubing supporting the sanple to be tilted slightly. The sample would tilt a similar anount. This led to the sample being tilted \pm 0.70 about two perpen- 
dicular axes. No effect of any $k$ ind was observed in the data traces recorded.

Based on these two studies it is felt that the symmetry problem did not arise from an inclination of the magnetic field relative to the plane of the samples.

Data exhibiting a similar lack of proper angular symmetry has recently been reported by latthey et al. (124) in white tin. In their data and the data presented here the signals not agreeing with the crystalline symetry are symmetric about the coil axes. one possible explanation is the unknown importance of the polarization of the rf fields incident on the sample's surface relative to the crystal axes in the sample. The solution may also be the angular relationship between the modulation field and the coil axis. This has been considered and no mechanism has been visualized, that would allow the modulation field information to feed through the system except the RFSE.

The symmetry problem in the data of ratthey et al. (124) occurred for a sample with a [001] sample normal (the axis in white tin for four fold symmetry). Examining their data and the data presented here have led to one condition in the two cases that is similar. The tin data with unexpected symetry were recorded when the magnetic field was rotated in a plane perpendicular to the axes of third zone pieces of the FS shaped like cylinders. Therefore; the rf electric field was 
always perpendicular to these cylinders. In their other data, the sensitivity to certain signals depended greatly on orientation of the rf field relative to these cylinders.

In the case of alaminum, the third zone FS is similar to four cylinders connected to each other around each square face of the BZ. The worst symmetry problem seen in the data presented here (Figure 42) occurred wen the rf electric field was perpendicular to two of these square face assemblies. In the remainder of the data taken the rf electric field was never perpendicular to any entire asgemblies. In some cases it was perpendicular to two of the four segments on two faces (Figures 43,44, and 45 and the [110] data). Presently no mechanisa is risualized to explain this problem based on the orientation of the rf fields relative to these cylinders. It is apparent from studying the data that two processes are interacting to yield the observed data. The process leading to the symetry problems is currently unexplained, but by taking complementary data the RFSE signals can be extracted.

In the raw data presented, the existence of complementing data resulting from sample rotations inside the coils made it possible to examine two sets of data for consistent data for both the [100] and [111] samples. This comparison of data made it possible to reject signal features that seem to result from the relationship botween the coil 
axis and the crystalline axes. It was also possible to elininate the line "A" at 500 Oe in Figure 42 because this feature did not scale wh changing sample thickness when preliminary data for another [100] sample were examined.

The data remaining after this examination have been used to determine FS calipers and will be discussed in a later section.

\section{B. Frequency studies}

Frequency study data were recorded for several samples with [100] and [111] sample normals. The only data shouing the frequency dependence of Equation 16 were obtained using Al 13 (a [100] sample normal). The data recorded for Al 12 showed erratic frequency dependence as will be seen later. These facts will be examined using the ideas discussed in an earlier section relating to frequency study problems. Consider the data for two samples vith [100] sample normals, Al 12 and Al 13. Recall from the discussion of samples with non-uniform thickness that the low field edge of the RPSE signal arises from the thickest part of the sample. From Figure 41 it is then seen that the onset will occur for $t=180$ microns for Al 12 and for $t=164$ microns for $A 113$ (ignoring the isolated point at 167 microns). Thus the corresponding features for Al 13 should be at field values about $10 \%$ higher than their counterparts for Al 12. This is seen to be true for the two traces shown in Figure 46 which were 


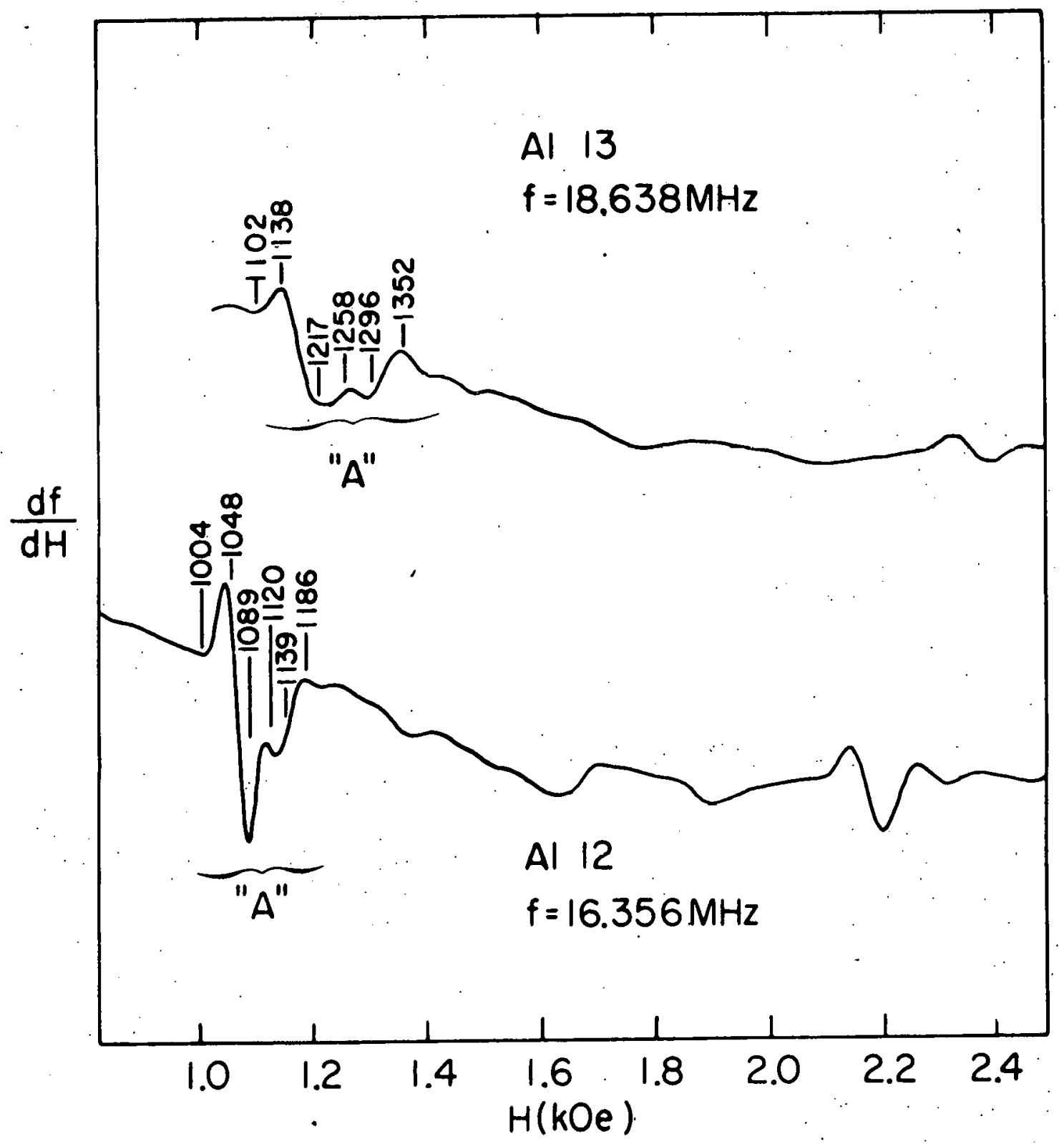

Figure 46. Data traces for Al 12 and Al 13 for the same crystalline direction using oscillator \#2 
taken for the same orientation of the magnetic field relative to the crystal axes.

Next consider the thickness distribution for the two samples as seen in Figure 41. It is seen that Al 12 has a smaller variation in thickness but also a ore erratic thickness distribution than seen for Al 13. The average and the standard deviation of the number of microscope readings per micron for every value of thickness between the minimum and maximum values have been calculated for these samples. The values for $A 112$ and $A 113$ are $1.89 \pm 1.56$ and $1.06 \pm 0.98$ respectively. This means that $A 113$ is more wedge-shaped than Al 12 and the behavior of the RFSE signals should be examined with this in mind. Consider feature "a" on the Al 13 trace in Figure 46 and the simplified signal behavior seen for a wedge-shaped sample in Figure 11b. Notice in Figure $11 \mathrm{~b}$ the first and last features are the same shape as each other but are inverted with respect to each other. This is the general behavior exhibited by feature "A" for Al 13. This trace also shous great structural.similarity to the Gantmahker (60, Figure 19) illustration of RFSE line splitting resulting from a wedge-shaped sample. Next consider feature "A" for $A 12$ and the curve seen in Figure 12 b. Peature "A" for $A I^{\prime} 12$ is seen to show more structure in its central region than its counterpart from Al 13. This is exactly the same beharior as seen when comparing Figure 12b for a 
wary sample with Figure $11 b$ for a wedge-shaped sample. If Al 13 is behaving like a vedge-shaped sample then the upper and lower features of signal "A" should result from the thinnest and thickest portions of the sample and the field values should be scaled accordingly. This can be expressed as

$$
B(\max t) / B(\min t)=(t \min ) /(t \max ) \text {. }
$$

The left hand side of Equation 25 can be evaluated from the RFSE data and the right hand side can be evaluated with the aid of Pigure 41. Prom Figure 46 it is seen that

$$
B(\max t) / B(\min t)=1138 / 1296=0.88
$$

and from Pigure 41 it is seen that

$$
\text { (t } \min ) /(t \max )=136 / 164=0.83 \text {. }
$$

The field values vere chosen as two corresponding features on the line shape of signal "A". The onset of the signals was not used in an effort to minimize any problem caused by the interaction of the two signals in the central region of the resonance. This numerical a greement is satisfactory in light of the unknown interaction of the two signals within feature "A".

Now consider the frequency study data taken for these two samples. Frequency study plots are shown in pigure 47. The data for Al 12 show no systematic frequency dependence. The lines for $A l 12$ have been examined using a linear leastsquares tit to the mathematical form of Equation 16. The 

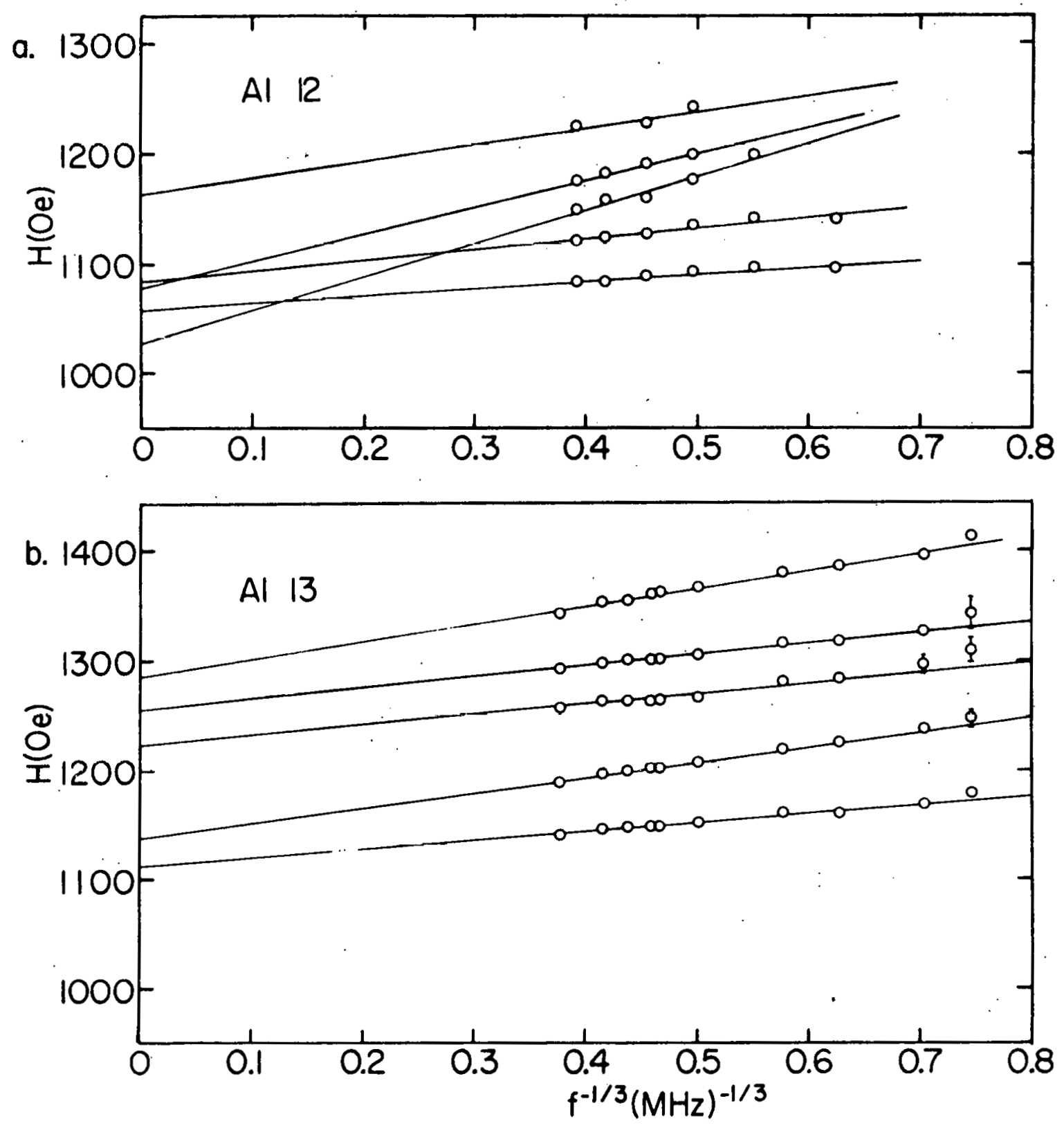

Pigure 47. Frequency stuay plots for Al 12 and al 13 for the same crystalline direction using oscillator \#2 
value of the exponents, $\alpha$, giving the smallest chi-squared for the five lines vere $0.09,0.07,1.61,0.17$, and greater than 2.4 starting with the lowest line of Figure $47 \mathrm{a}$. This behavior is not consistent from line to line and the data have been considered to be of no value for a useful frequenzy study. The data for al 13 examines the frequency dependence of feature "A" on Figure 46. Examine lines (1 and 2) and (4 and 5). These lines correspond to features presumed associated with the RPSE signal arising from the thickness extremes. Line 3 vill be ignored to avoid posibile problems in the central region of the signal where the tro signals might be interacting. The data for lines 1, 2, 4, and 5 were examined for compatibility ith the frequency dependence expressed in Equation (16) using a linear least-squares fit program and the cleveland method for determining proper field values.' Both of these techniques have been discussed in detail in a preceding section.

The linear least-squares examination of the data led to the information found in rable 3. The first tro colums give the line number and the value of the exponent, $\alpha$, corresponding to the swallest value of chi-squared for each line. The exponents for the two lines in each pair are in good agreement. For each pair of lines a value of the exponent was selected to represent the real exponent for the tro lines, $\alpha($ real). These values and the corresponding line 
intercepts and slopes are given in the last three columns of the table. These data for al 13 show good frequency dependence for the signals arising from both sample thickness extrema. This is to be expected since at each extremum for a uedge sample, only the skin depth can change to affect the width of the RFS signal.

Table 3. Al 13: Linear Least squares fit results

\begin{tabular}{|c|c|c|c|c|}
\hline Line & $\begin{array}{l}\text { Best fit } \\
\text { exponent }\end{array}$ & $\begin{array}{c}\text { Selected real } \\
\text { exponent } \\
\alpha(\text { real })\end{array}$ & $\begin{array}{c}\text { Intercept } \\
\text { (Oe) }\end{array}$ & $\begin{array}{c}\text { Slope } \\
\text { Oe }(M H z) 1 / 3\end{array}$ \\
\hline 1 & 0.46 & 0.48 & 1125 & 73 \\
\hline 2 & 0.51 & 0.48 & 1160 & 128 \\
\hline 4 & 0.49 & 0.46 & 1269 & 95 \\
\hline 5 & 0.45 & 0.46 & 1310 & 147 \\
\hline
\end{tabular}

The results of the cleveland technique for finding the proper exponent for each pair of lines are 0.19 for lines 1 and 2 and 0.17 for lines 4 and 5 . The corresponding intercepts for two sets of 1 ines are 1082 oe and 1201 je respectively.

The numerical results for these frequency study data can now be analyzed using the ideas previously dereloped for wedge-shaped samples. The comparisons should be realistic 
because the frequency range for the data generated for the earlier discussion is identical to the frequency range for the Al 13 data. Pirst consider the ratio of the "cleveland best fit exponent", $\alpha(\min )$, to the selected real value of the exponent for each set of lines, $\alpha$ (real). For. Iines 1 and 2 this yields 0.40 and for lines 4 and 5 it yields 0.37 . These values can be used with Pigure 13 to obtain an indication of the relative importance of skin depth broadening and thickness variation broadening for the sample. Prom pigure 13 it is seen that the value of RATIo deduced for lines 1 and 2 is between 0.62 and 0.81 . Similarly for lines 4 and 5 the range for RATIO is 0.70 to 0.91 .

RATIo can also be found directly from the lines of the frequency study as discussed earlier. Recall that Rato is defined as the largest spread in intercepts for a family of lines divided by the largest difference in slopes for the same lines. These values are taken from the lines wen they are plotted on an axis system based on the real value of the exponent for the family of 1 ines. Osing this definition it is seen that for 1 ines 1 and 2 ,

RATIO ( 1 and 2$)=35 / 55=0.64$.

Similarly for lines 4 and 5 ,

$$
\text { RAT IO }(4 \text { and } 5)=41 / 52=0.79 \text {. }
$$

Both of these values are seen to fall in the anticipated range found from Pigure 13. It is also vorth noting that 
based on the values of $\alpha($ min) $/ \alpha($ real) for these tro sets of lines and Figure 13, the value of RATIO (1 and 2) is expected to be less than RATIO (4 and 5). This is seen experimental$1 Y$

Another comparison between the experiment and the theoretical predictions on this matter can be made concerning the amount that the cleveland method reduces the intercept for a family of lines. Using the data on the individual lines from Table 3 and the common intercepts for the two series of lines for their respective values of $\alpha(\mathrm{min})$ it follors that $(A(1, \alpha($ rea 1$))-A($ ave $)(1$ and $2, \alpha(\min )))=1125-1082=43$ De

and $(A(4, \alpha($ real) $)-A(\operatorname{ave})(4$ and $5, \alpha(\min )))=1269-1201=68$ oe. (31) The theoretical predictions can be based on the spread in the intercepts for the two series of lines for their appropriate values of $\alpha($ real).

Intercept spread $(1$ and 2$)=1160-1125=35^{\circ}$ oe (32)

Intercept spread (4 and 5) =1310-1269=41 0e.

The value of (A(real)-A(min)) is expected to be roughly equal to the spread in intercepts for the lines as discussed earlier. Thus it would be expected that for Iines 1 and 2 the intercept will be reduced about 35 oe and for lines 4 and 5 the reduction will be about 41 0e. From these two cases it is seen that the qualitative agreement is good. The ratios of the experimental to theoretical reductions in the intercepts for the tro sets of lines are: 


$$
\begin{aligned}
& \text { Lines } 1 \text { and } 2:(43 / 35)=1.23 \\
& \text { Lines } 4 \text { and } 5:(68 / 41)=1.66
\end{aligned}
$$

In both cases the reductions were larger than expected. The largest reduction was predicted and observed for lines 4 and 5 rather than for lines 1 and 2 .

The theoretical concepts examined earlier for wedgeshaped samples have been examined as they relate to experimental frequency study data. The idea of signal splitting due to a vedge-shaped sample has been substantiated experimentally. It has also been noted that each of these split signals demonstrates the frequency dependence expected from good, flat samples.

The effects of redge-shaped samples on the cleveland method for determining reson ance field values have been compared with the theoretical predictions and good qualitative agreement has been found.

C. Fermi surface Calipers and orbit Assignments

Fermi surface calipers have been determined from the raw RFSE angular data recorded and the thickness of the samples used. Mhere symmetry problems occurred in the angular data, complementary data have been recorded to allow systematic examination of both data sets to determine which features are common to both. These features have then been used to determine FS calipers resulting from RPSE. 
The calipers have been examined using the computer predictions for second-zone calipers and the conputer FS contour overlay plots to determine the origins in $k$-space of the various signals. The angular ranges and dependences for the experimental signals will be compared with those resulting from the NFE computer results.

The caliper data will be presented only on a rectangular coordinate system of caliper versus angle. polar plots have not been used because for the second-zone hole surface of aluminum the caliper dimensions do not occur in a common plane. Thus the calipers do not correspond to FS dimensions in a single plane perpendicular to the normal. This problem in data interpretation has been discussed in nore detail in an earlier section on the RFSE. As a result of this problew for aluminum the calipers have been plotted on rectangular coordinated to facilitate an easy comparison to the signal predictions resulting from the computer calculations.

Comparison of the experimental calipers will be made. with the NFE second-zone hole surface. Recall that the NFE second-zone surface is less complicated than the actual second-zone FS. The difference in the two Fermi surfaces is the rounding of sharp edges on the actual surface resulting from interactions neglected in the NFE model. Estimates of rounding effects will be made and used to adjust the NPE calipers for further comparison with the experimental 
results.

Estimates of rounding can be made using the results of Pippard (42) and Kamm and Bohm (36). These studies yielded information on rounding on two different types of edges on the second-zone PS. Pirst examine the work of Pippard and designate the edge considered as Type I. Figure 48a shous a second-zone cross section in a (100) plane through the center of the BZ. The corner examined by pippard is located at the symetry point $w$ and is indicated in the figure. The rounding estimate made at this corner is also used for the edge of the second-zone fS between the symmetry points 1 and D. This rounding occurs at the intersection of a FS edge common to two hexagonal faces and a square FS face perpendicular to a [010] axis. Otilizing his information (42, Pigure 11) a radius can be deduced for the rounding, $r=0.06 \AA^{-1}$. This leads to a reduction of $0.04 \AA^{-1}$ in the distance from the center of the $\mathrm{BZ}$ to the corner being examined. Edges between square and hexagonal faces on the second-zone FS will be referred to as. Type $I$ edges in the remaining discussion. The remaining second-zone FS edges are comon to two hexagonal faces and will be referred to as rype II edges. Data on a Type I edge can be taken from Kamm and Bohm (36, Figure 4). Their data relate to the rounding of a Type II FS edge where it intersects a square face perpendicular to a [010] axis. 
a.
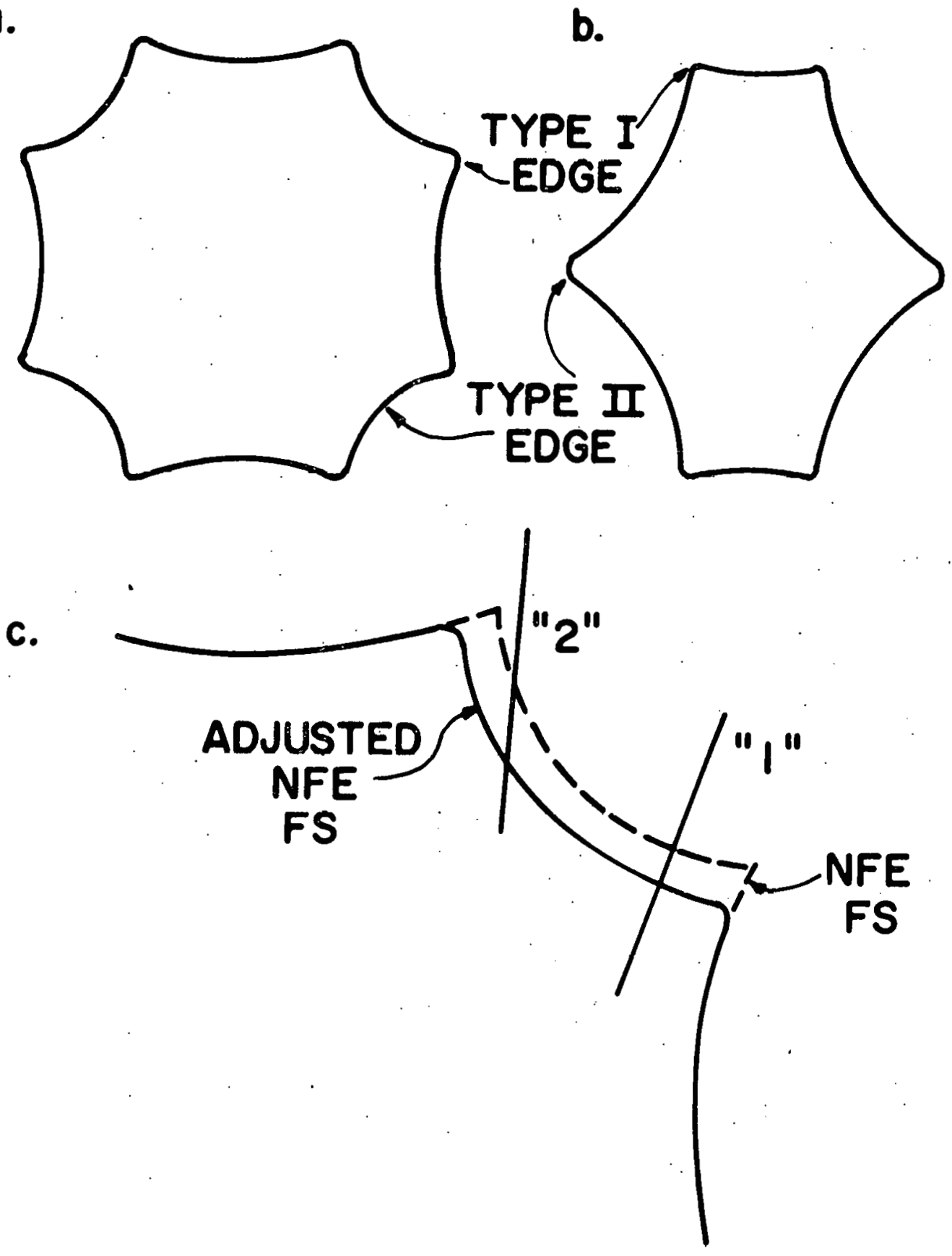

Figure 48. Examples of FS rounding which modifies the NFE second zone FS 
Such an edge is labeled in Pigure $48 \mathrm{~b}$ wich illustrates the intersection of the NFE second-zone PS with a (110) plane through the center of the $\mathrm{BZ}$. The indicated rounding decreases the distance from the zone center to the Fs edge by roughly $0.12 \AA-1$.

Figure 48c illustrates how the caliper reduction can depend on the orientation of the electron orbit on the PS. orbit "1" is perpendicular to the rounded edge and the caliper reduction is minimized. Orbit.n" makes an acute angle with the rounded edge and the caliper reduction is larger than seen for orbit "1".

It should also be noted that electron orbits crossing Type I edges must be considered carefully to see if the caliper reduction is appropriate. Consider an electron following.a real space trajectory with the shape seen in figura 48a. If the sample normal is along a cubic axis the rounding of the Type I edge does almost nothing to reduce the caliper. But if the sample normal passes through the indicated Type I edge, then the entire rounding contributes to the reduction of the FS caliper.

The data will now be discussed for [100], [110], and [111] sample normals in that order. The calipers and orbit assignments vill be discussed in detail and then sumarized for each sample. 


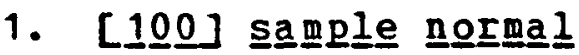

The angular data used vere taken using sample Al 12. Due to the lack of any frequency dependence for this sample, the resonance value of the magnetic field to be used in Equation 9 has been taken at the low-field end of the resonance feature. The low-field end of the resonance occurs for the thickest part of the sample so the thickness used in Equation 9 vas 180 microns as seen in Pigure 41.

Figure $49 a$ contains the calipers determined in this study. Figure $49 \mathrm{~b}$ shows the predicted calipers for this sample for the NFE second-zone model.

From the NFE model caliper "A 1 " is seen to have the values $2.69 \AA^{-1}$ at $0^{\circ}$ and $3.01 \AA^{-1}$ at $20^{\circ}$. The signal results from complete orbits around the second-zone FS as indicated in Pigure 50. These orbits cross two Type I edges in a manner that is not expected to to significantly reduce the calipers. Experimental calipers for "n1" were $2.78 \%-1$ at $0^{\circ}$ and $3.038^{-1}$ at 200 . Beyond 200 the experimental signal became too weak to measure. The loss of signal strength is expected as seen in Figure $49 \mathrm{~b}$ for caliper "A1". Agreement is within $3 \%$ at $00^{\circ}$ and $1 \%$ at $20^{\circ}$.

Caliper "Bl" can be examined at $15^{\circ}$ for the tro figures in a similar manner. The NFE caliper is $2.79 \AA^{-1}$. The hole orbits contributing to this signal are indicated and labeled in Figure 50. Notice that these orbits cross two Type I.I 
o.
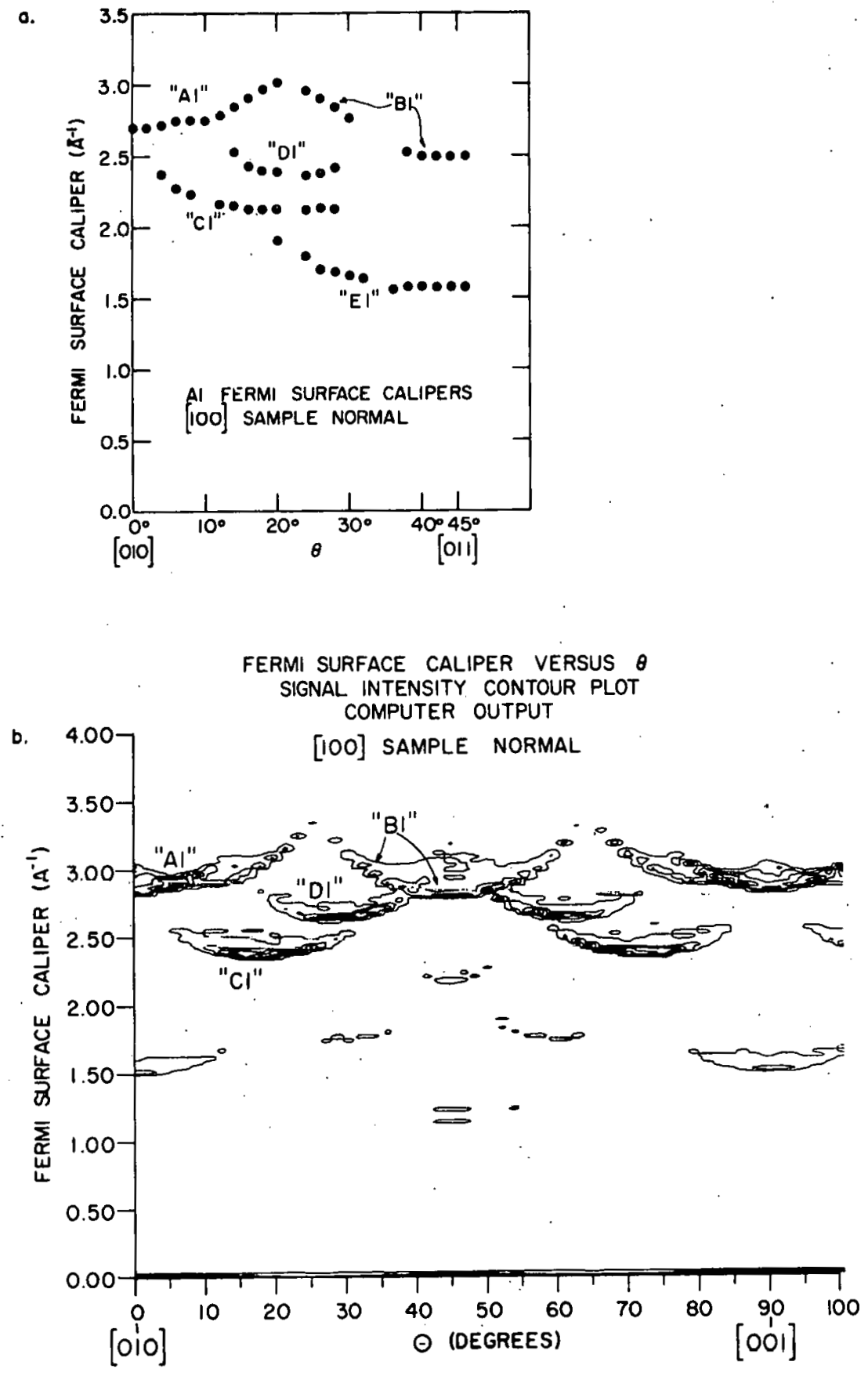

Figure 49. Experimental and theoretical aalipers datermined for a [100] sample normal 


\section{AL SECOND ZONE FERMI SURFACE CROSS SECTION OVERLAY PLOT COMPUTER OUTPUT}

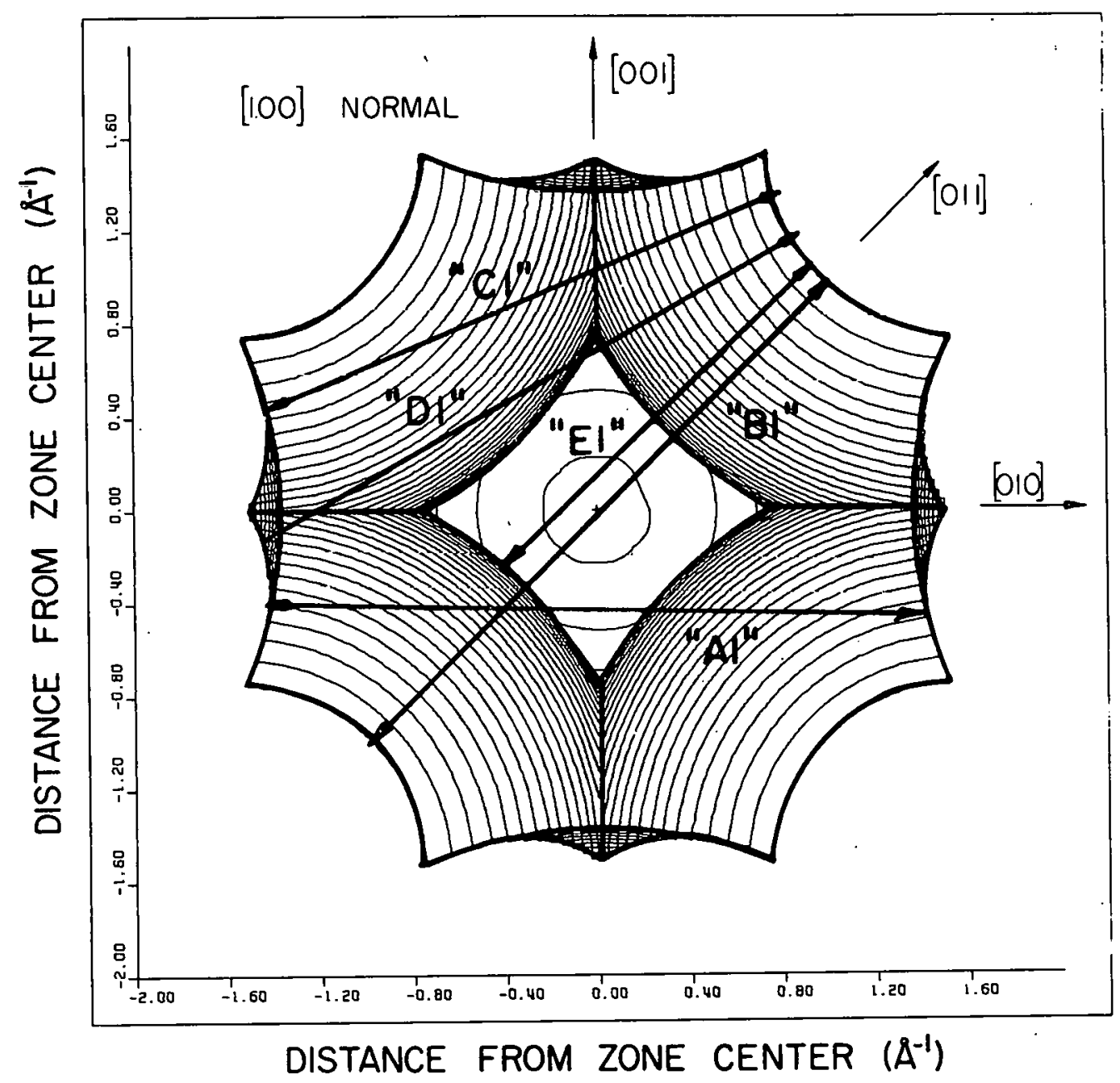

Figure 50. Orbit assignment.s for experimental calipers for a [100] sample normal 
edges. Thus the caliper for this orbit will be reduced by at least two times the value deduced from the data of Kam and Bohm. If the plane of the orbit does not intersect the Type II edge at right angles, then the caliper will be even further reduced due to the geometry involved. This is based on the assumption that the rounding seen by Kam and Bohm applies for the entire edge comon to tro heragonal faces and not just where the edge meets the square PS face. Utilizing the Type II rounding reduces the expected caliper to $2.55 \AA^{-1}$. The experimental value of $2.49 \AA^{-1}$ agrees to within $2 \%$.

It should be noted that the calipers "A1" and "Bin" appear to intersect in the region of $20^{\circ-23^{\circ}}$. Theoretically this is expected to occur at about $25^{\circ}$. The edge rounding just examined vould shift this intersection to a smaller angle as can be seen from Figure 50. Scaling the change in location of the edges on Figure 50 indicates the peak should be shifted about 30 toward smaller angles.

Between $30^{\circ}$ and $38^{\circ}$ the signal associated with caliper "B1" broadens and is not distinct enough to allow a measurement. This is not anticipated from the computer predictions of signal intensity. The veakening may be related to the interaction of signals "Bl" and "Dl" in this angular range.

Caliper "Cl" follows the same angular range on both the predicted and observed data. This caliper results from a signal that does not have an easily selected low-field edge. 
This fact contributes to some of the scatter in this data. The angular dependence can be inferred from the next higher feature on the data trace which is also associated with this signal. Its minima is rather broad and occurs between $16^{\circ}$ and $23^{\circ}$. This is compatible with the minima seen on Figure 24 which is broad and occurs between $15^{\circ}$ and $22^{\circ}$. The electron orbit giving rise to caliper "Cl" is indicated in Figure 50. It is seen that this orbit crosses one Type I edge in a direction that will contribute very little to a caliper reduction. It also crosses a Type II edge which will cause a reduction of about $0.12 \$^{-1}$ in the expected caliper. Therefore, the NFE caliper at the minima is $2.318-1$ and the adjusted NFE value is $2.19 \AA^{-1}$. The experimental value at the minima is $2.12 \%-1$ which agrees to within $3 \%$.

Caliper "D1" on Pigure 49 a falls belov its expected magnitude and also appears shifted to smaller angles than expected. The orbit thought to be contributing to this signal is shown in Figure 50. This orbit crosses the same edge conbination as orbit "Cin so the rounding adjustaents to the NFE caliper is expected to be $0.12 \AA^{-1}$. The resulting NFE and adjusted NFE calipers at the minima $\left(27^{\circ}\right)$ are $2.56 \AA^{-1}$ and $2.44 \AA^{-1}$ respectively. The experimental minima of 2.36 occurs at $24^{\circ}$. The agreement is vithin $3 \%$.

No other signals have been identified in the complementing data sets that result from complete orbits 
around the second-zone hole surface. Calipers near $1.50 \AA^{-1}$ at 00 in Figure $49 \mathrm{~b}$ were not found but these signals are predicted to be much weaker than the signals already discussed. Some signals have been attributed to partial orbits on the second-zone hole surface. Signals of this type have been discussed by Gantmahker (60) and have been predicted in aluminum by Druyvesteyn and smets (123). They occur when an electron on an orbit moves parallel to the sample's surface at points other than extrema on the FS. If the electron orbit is such that it moves parallei to the surface at each surface, it can yield a RPSE signal even though it does not complete a full orbit. The important factor is for the particle's motion to remain near the surface long enough to interact with the applied fields. Examples of three partial orbits are shown in Figure 51. These regions on the Fs can easily be selected in the computer FS contour overlay plots. The electron's velocity at a point on the FS is always perpendicular to the FS at the point, so velocities parallel to the surface of the sample will occur for ps regions where the FS is paraliel to the normal axis. on the contour orerlay plots the normal axis is perpendicular to the page. Thus regions were contour lines are very dense vill correspond to the particle's velocity being almost parallel to the sample's surface. Such a situation exists in Figure 50 on the four sides of the square face centered on the [100] axis, so it is 

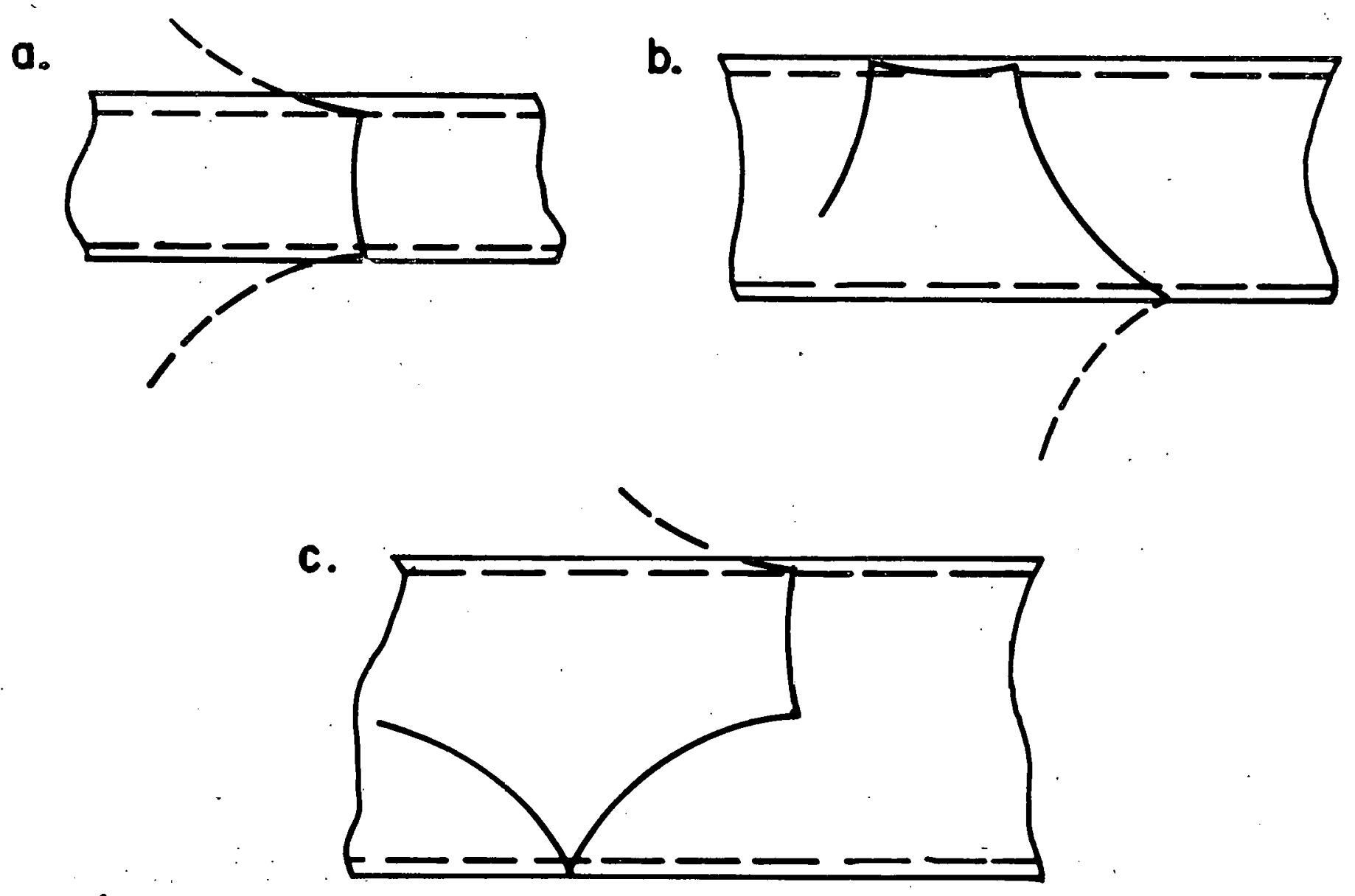

Figure 51. Examples of partial orbits that can contribute to RPSE signals 
possible to use the contour overlay plots to great advantage when evaluating possible contributions from partial orbits. Caliper "E1" on Figure $49 \mathrm{a}$ will be compared to the caliper resulting from the partial orbit "E1" on Figure 50. This orbit is qualitatively sketched in Figure 51c. From the NFE model in Figure 50 it is seen that caliper "E1" should contribute signals over the angular range of 190 to $45^{\circ}$ and it crosses a Type I and a Trpe II edge at the limits of its real space motion. The Type I edge crossing is not expected to cause a caliper reduction due to the geometry involved. Notice that as the angle decreases toward 200 , orbit "E1" begins to include motion across the square face near one of the sharp NFE corners. This corner is the same one studied by Kamm and Bohm so this caliper vill be reduced by a second Type. II correction in this angular region. The Type II edges vill cause a caliper reduction as discussed earlier. The NFE calipers are $2.478^{-1}$ at 190 and $1.868^{-1}$ at $45^{\circ}$. Including rounding effects reduces these to $2.25 \AA^{-1}$ and $1.75 \AA^{-1}$ respectively.

The experimental calipers designated "E1" cover the same angular range but their values fall below the expected values. They vary from $1.90 \AA^{-1}$ at $20^{\circ}$ to $1.56 \AA^{-1}$ at $45^{\circ}$. The experimental values are $15 \%$ and $10 \%$ too small at $20^{\circ}$ and $45^{\circ}$ respectively. The differences in magnitudes are not understood. Effects of rounding have already been included in 
finding the expected values and the caliper differences are much too large to attribute them to further refinements in the rounding picture. If adjustments in the rounding model were made to account for this difference, the agreement for calipers already discussed would be destroyed. So no reasonable adjustments are seen and the differences remain unexplained. For this reason it is considered unlikely that this assignment is correct. But a careful examination of the FS has been made and another explanation has not been found for these data.

A low-field feature exists in the data sets presented in Figures 42 and 43 that also does not agree with a caliper that wight be reasonably expected from the seçond zoné. There is some disagreement between the complementing data sets for angles less than 100. This feature shous the form expected for an orbit crossing the square face on the [ 1.00 ] axis but the caliper observed for $45^{\circ}$ is about $30 \%$ smaller than expected from an examination of the contour overlay plot. Examination of the recorded data for this feature reveals that it does not have a line shape generally seen in the RFSE. In fact it appears to be associated with the background curve for $(d f / d H)$ versus $H$. The feature corresponds to the region where the slope of the background changes from positive to negative. For these reasons it is felt that these features are related to the processes governing the 
background curve and not electron orbits spanning the sample's thickness. Therefore a caliper based on this feature is not shown.

Figures 42 and 43 also show several regions of high signal content for $\theta=45^{\circ} \pm 10^{\circ}$ and magnetic field values of 600 to 750 oe and 900 to 1100 oe. Some of these detailed variations mày result from the small third-zone ps aras but the signals are too broad to allow an adequate treatment of the data to resolve the question.

The lack of symetry seen in Pigures 42 and 43 has made caliper assignnents very difficult except for the strongest signals. The distortions placed on the normal four-fold symmetry have reduced the number of signals considered for caliper evaluation. It is very possible that several valid signals have been ignored because of their interaction with other signals not possessing the proper symetry.

Further complications have ben added resulting from samples that vere not as flat as desired. The signal broadening caused by this has contributed to signal overlap in many regions and reduced the clarity of the angular dependence in such regions.

Caliper values and a comparison of the theoretical and experimental values are sumarized in table 4. 
Table 4. Caliper results for [ 100 ] sample normal

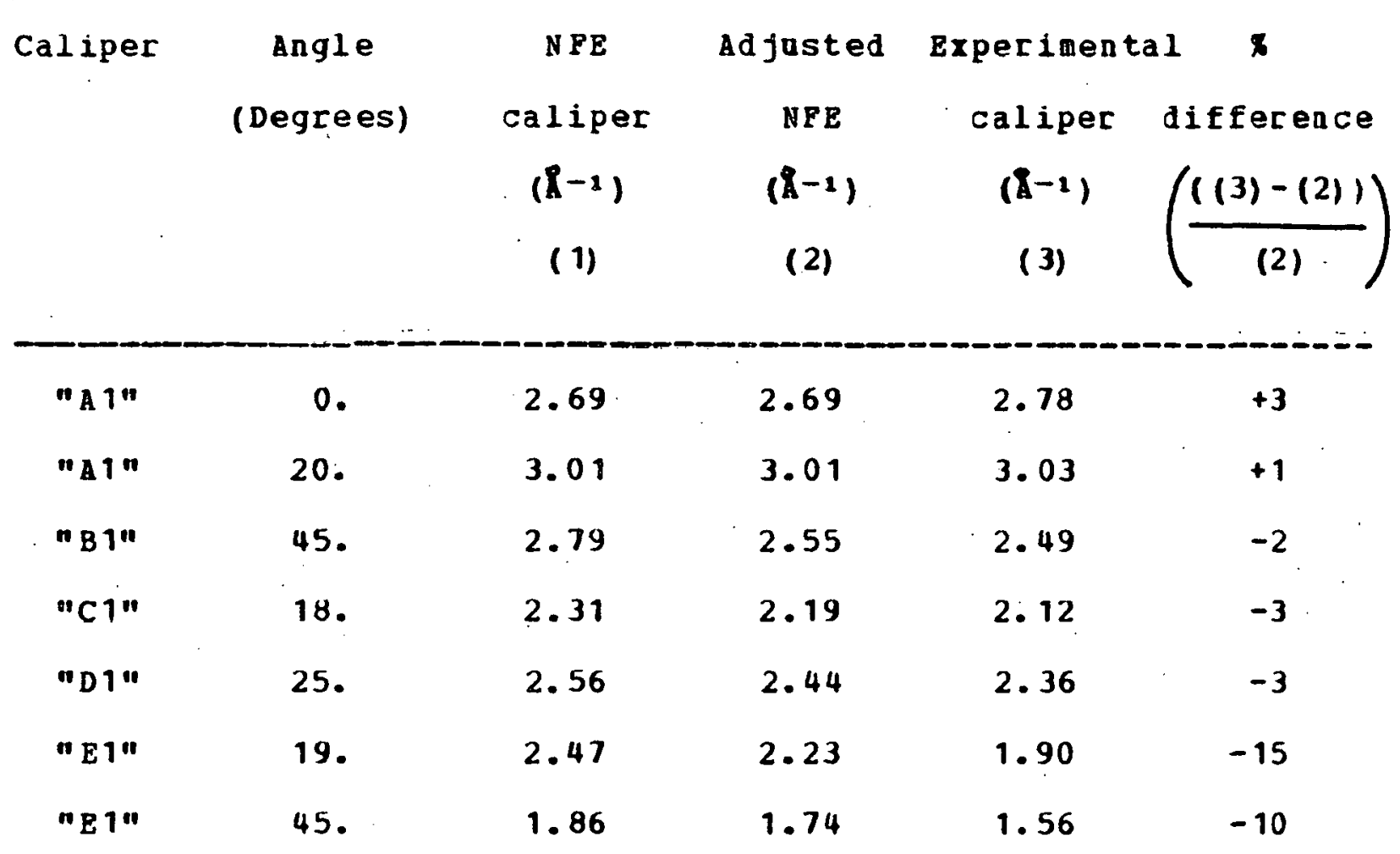

\section{2. [110] sample 므오므므}

FS calipers vere determined using the angular data recorded with sample Al 7. The signals observed were very veak. For this reason the data do not represent all the signals expected for this sample orientation. The data seen have been related to expected signals as seen from the computer predictions for a [110] sample normal.

Due to the veakness of the signals it was very difficult to determine the low-field edge of the signals. For this reason the resulting PS calipers may be slightly larger than 
the actual values. The error could be on the order of 0.10h-1. This would cause a shift to smaller calipers but the angular dependence of the signals relative to each other nould not be altered.

The caliper magnitudes have been calculated using Equation 9. The sample thickness taken from Pigure $41 \mathrm{c}$ is 196 microns. The isolated reading of 198 microns has been neglected.

The angular variations of the calipers are shoun in Figure 52a. They will be compared uith the computer predictions for calipers of the second-zone hole surface as seen earlier in Figures 25 and 28 .

Caliper "\$2" from Pigure 52 a corresponds to the upturned signal shape seen at $0^{\circ}$ and about $2.18-1$ on Figure 52 b. This signal is attributed to the hole orbit indicated in Figure 53. The orbit crosses two Type I edges in a direction that should result in corrections close to the full estiated value of $0.04 \AA^{-1}$ for each edge. The NFE and ajusted NPE calipers are then $2.098^{-1}$ and $2.01 \mathrm{~A}^{-1}$ respectively at 00 . The experimental caliper is $2.08 \AA^{-1}$ and agrees with the adjusted NPE value within $3 \%$.

Prom Pigure 52b it is seen that a smooth extension of this signal intersects a higher caliper signal, "B2n, at about $13^{\circ}$. This is compatible with a smooth axtrapolation of the calipers "A2" and "B2" in Figure 52 . 
a.
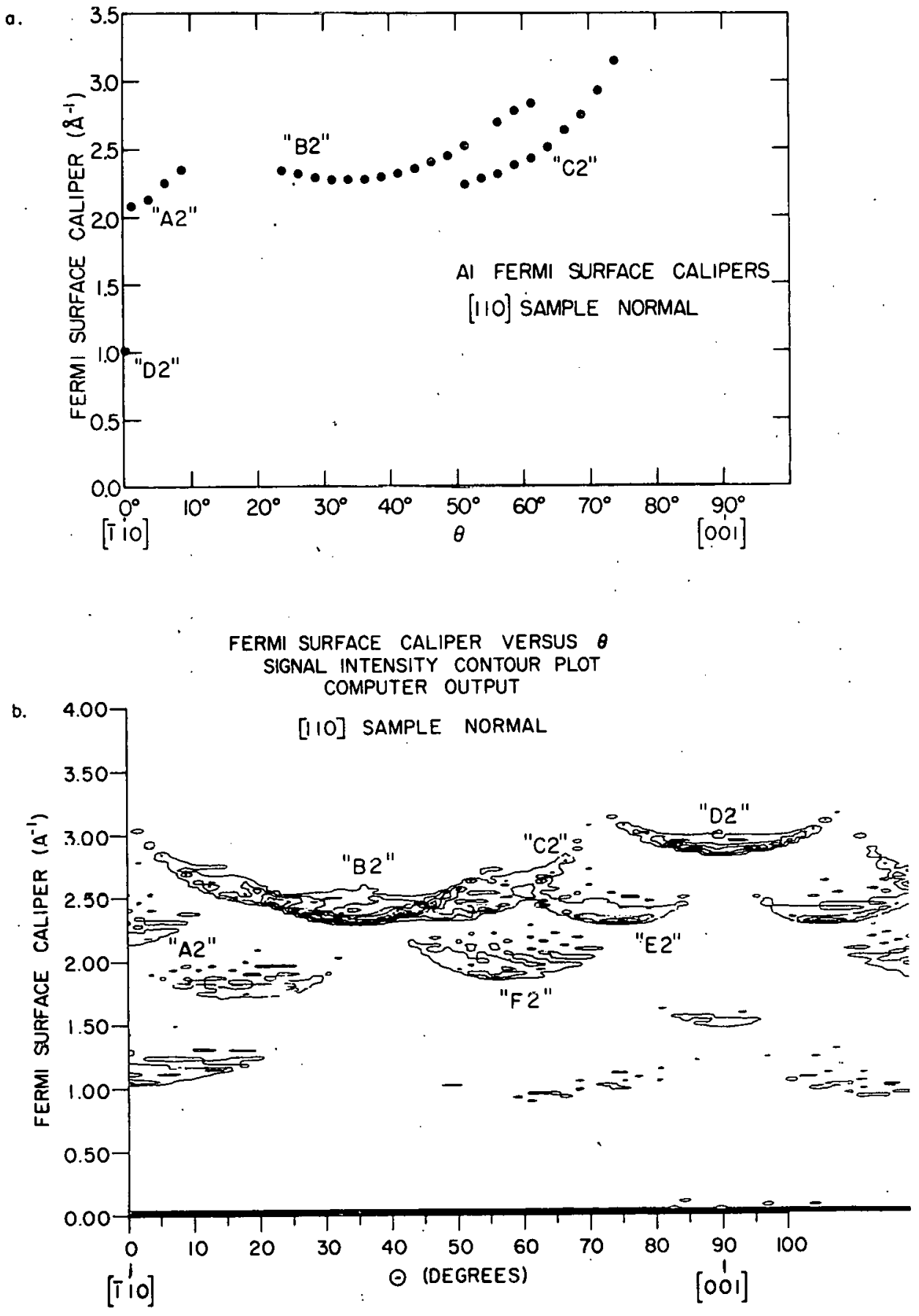

Figure 52. Experimental and theoretical calipers determined for a $[110]$ sample normal 


\section{AL SECOND ZONE FERMI SURFACE CROSS SECTION OVERLAY PLOT COMPUTER OUTPUT}

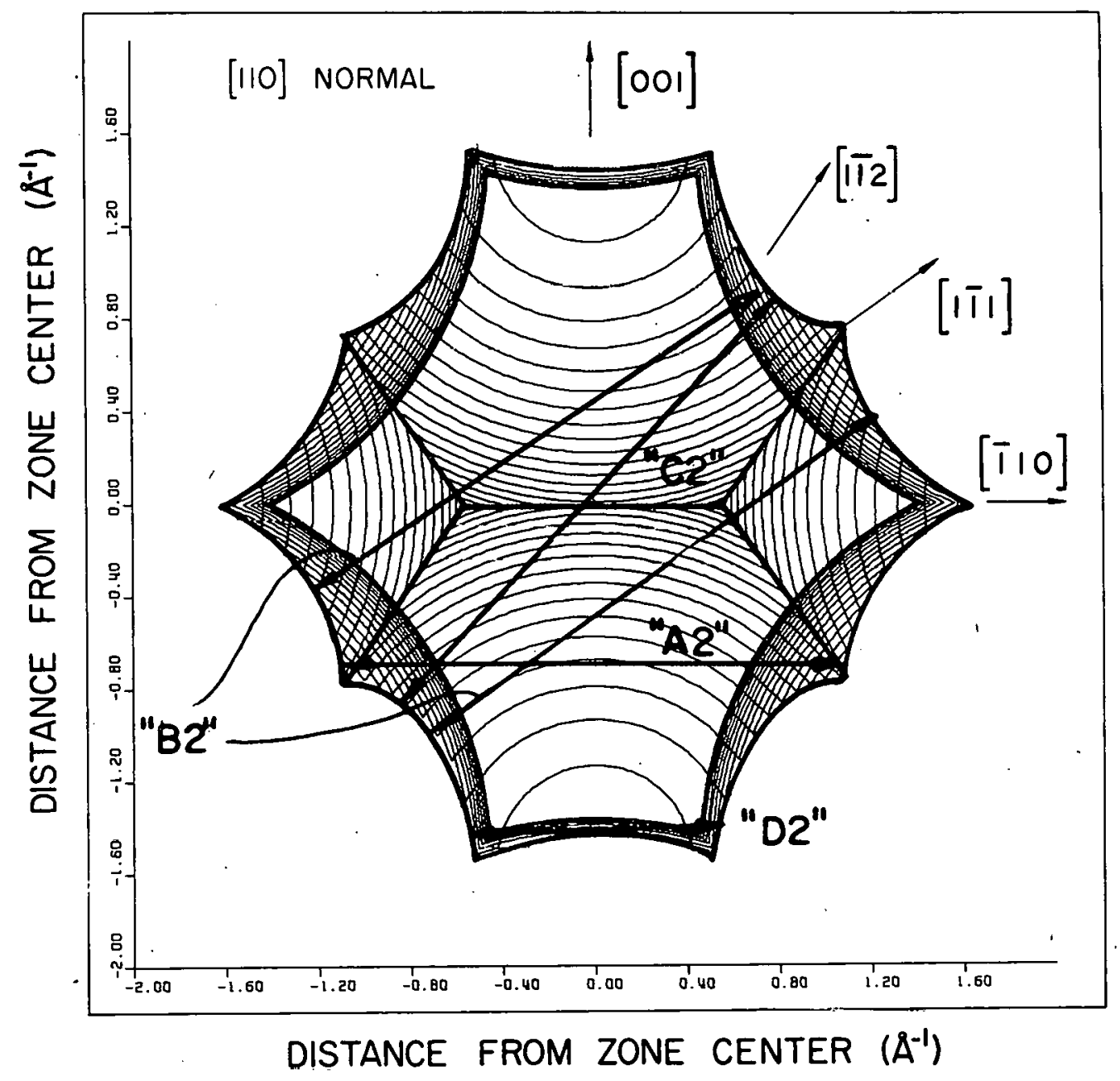

Figure 53. Orbit assignments for experimental aalupers for d [110] sample normal 
Next examine caliper "B2". Prom Figure 52b it is seen that the predicted intensity of this signal is fairly low until an angle of about $20^{\circ}$ is reached. Signal "B2" from the experimental data was not observed for angles less than $24^{\circ}$. From Pigure 53 it is seen that both "B2n orbits cross one Type I edge and one Type II edge. The Type I edge is the same as discussed above and a caliper reduction of $0.04 \mathrm{~A}^{-1}$ is expected. The Type II edge rounding is not very effective at reducing the caliper due to the orbit geometry. The estimated reduction of $0.05 \mathbb{R}^{-1}$ is based on a graphical examination of the edge under consideration. The net correction is then about $0.09 \AA^{-1}$.

The NFE and adjusted NEE calipers for "B2" at $240^{\circ}$ are $2.34 \AA^{-1}$ and $2.25 \$^{-1}$ respectively. The experimental value of 2.36\%-1 agrees to within 5\%. A sinilar examination of NFE, adjusted NFE, and experimental values at the minima for "B2" yield $2.25 \AA^{-1}, 2.16 \AA^{-1}$, and $2.27 \AA^{-1}$ respectively. The agreement is again within 5\%. Both the experimental and theoretical minima for "B2" occur at $34^{\circ} \pm 1^{\circ}$. Based on the NFE second zone it is seen that caliper "B2" rises to $2.65 \AA^{-1}$ near $55^{\circ}$. It then remains constant between $55^{\circ}$ and $63^{\circ}$. The adjusted caliper value is about $2.56 \AA^{-1}$. The experimental data shows signs of flattening out between $55^{n}$ and $61^{\circ}$ but the curve never does become flat. The value at $60^{\circ}$ is $2.83 \AA^{-1}$. The values differ by $11 \%$. 
An examination of caliper "B2" on figure 52 b shows that a lower branch splits a wa from "B2" as $\theta$ increases beyond 430. This signal, "C2n, is then seen to extend upward toward 3.13\%-1 at $72^{\circ}$. Orbit "C2n crosses tro Type II edges in the same manner just considered for "B2". Thus the rounding is expected to reduce the caliper by $0.108^{-1}$ to $3.038-1$ at 720 . Such a signal was observed experimentally. The caliper "c2" extends to roughly $74^{\circ}$ at $3,15 \AA^{-1}$ and agreenent is vithin $4 \%$. The hole orbit yielding signal "c2" is labeled on Figure 53.

A signal for the caliper "D2" on Figure 52 a was observed but an angular study was not made in this field range. This feature corresponds to the expected signal "D2" seen on Figure 52b near $0^{\circ}$ and $1 \AA^{-1}$ and the hole orbit is indicated in Figure 53. The orbit crosses two Type II edges and the reduction in caliper is expected to be the same as found for orbit "C2", 0.108-1. The NFE and adjusted NFE calipers are then $1.00 \AA^{-1}$ and $0.90 \mathbf{R}^{-1}$ respectively. The experimental value of $0.95 \AA^{-2}$ agrees to within $4 \times$ with the adjusted NFE val ue.

Signals "B2" and "F2" on Figure 52 bere not observed even though they are expected to be fairly strong. This is a direct result of the experifental sensitivity for the RPSB being zero for $90^{\circ}$ in Figures $52 \mathrm{a}$ and $52 \mathrm{~b}$.

Recall that due to the veakness of the signals it vas extremely difficult to determine the low-field feature of the 
signals. Por this reason the experimental values for the calipers should probably be slightly swaller than those indicated above.

The agreement seen in the angular behavior is of importance. The comparison of the expected and observed calipers showed maxima, minima, and signal splittings at the proper angular locations. All the signals seen for this sample agreed ith predicted calipers and were due to complete hole orbits around the second-zone hole surface. No signals vere seen that have not been discussed.

Due to the reakness of the signals an attempt vas not made to rotate the sample in the coil and take data on the region near $90^{\circ}$ in Figures, $52 a$ and $52 b$.

Caliper values and a comparison of the theoretical and experimental values are sumarized in rable 5.

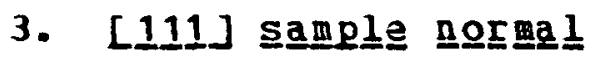

Several [111] samples were used to obtain data. Signals vere observed whose field values varied with sample thicknesss as required by Equation 9. The data to be discussed vere recorded using Al 16. This sample yielded the largest number of signals and the best signal strength of the samples examined. Again complementary data sets (Figures 44 and 45) have been used in an effort to select useful data and to eliminate data whose symmetry does not agree with the FS symetry. Calipers have been calculated using a sample 
Table 5. Caliper results for [110] sample normal

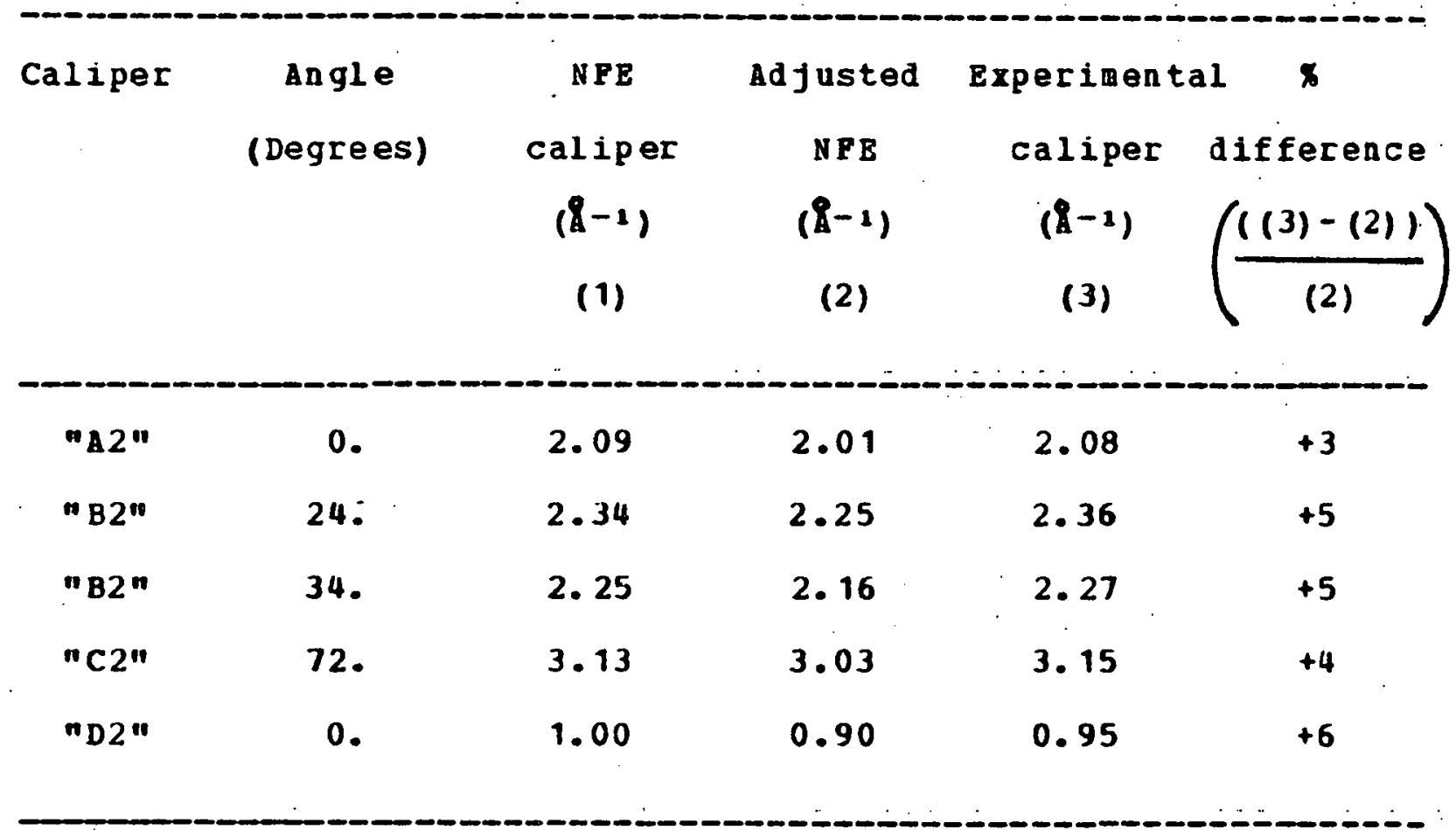

thickness of 120 microns as taken from Figure $41 \mathrm{~d}$.

Caliper "A3" on Figure 54 a has been attributed to holes completing motion across a heragonal second-zone FS face perpendicular to the normal axis. This partial orbit is illustrated and labeled as "a 3" in Figure 55 and is similar to that sketched in Figure $51 \mathrm{~b}$. The caliper varies smoothly over the angular range from $6^{\circ}$ to $30^{\circ}$.

The orbit crosses one. Type II edge but the geonetry involved for this sample decreases the caliper reduction due to rounding on this edge. Prom a graphical examation of a Type II edge for this orientation, the caliper reduction is estimated to be about $0.02 \AA^{-1}$. It also crosses one Type I 
o.
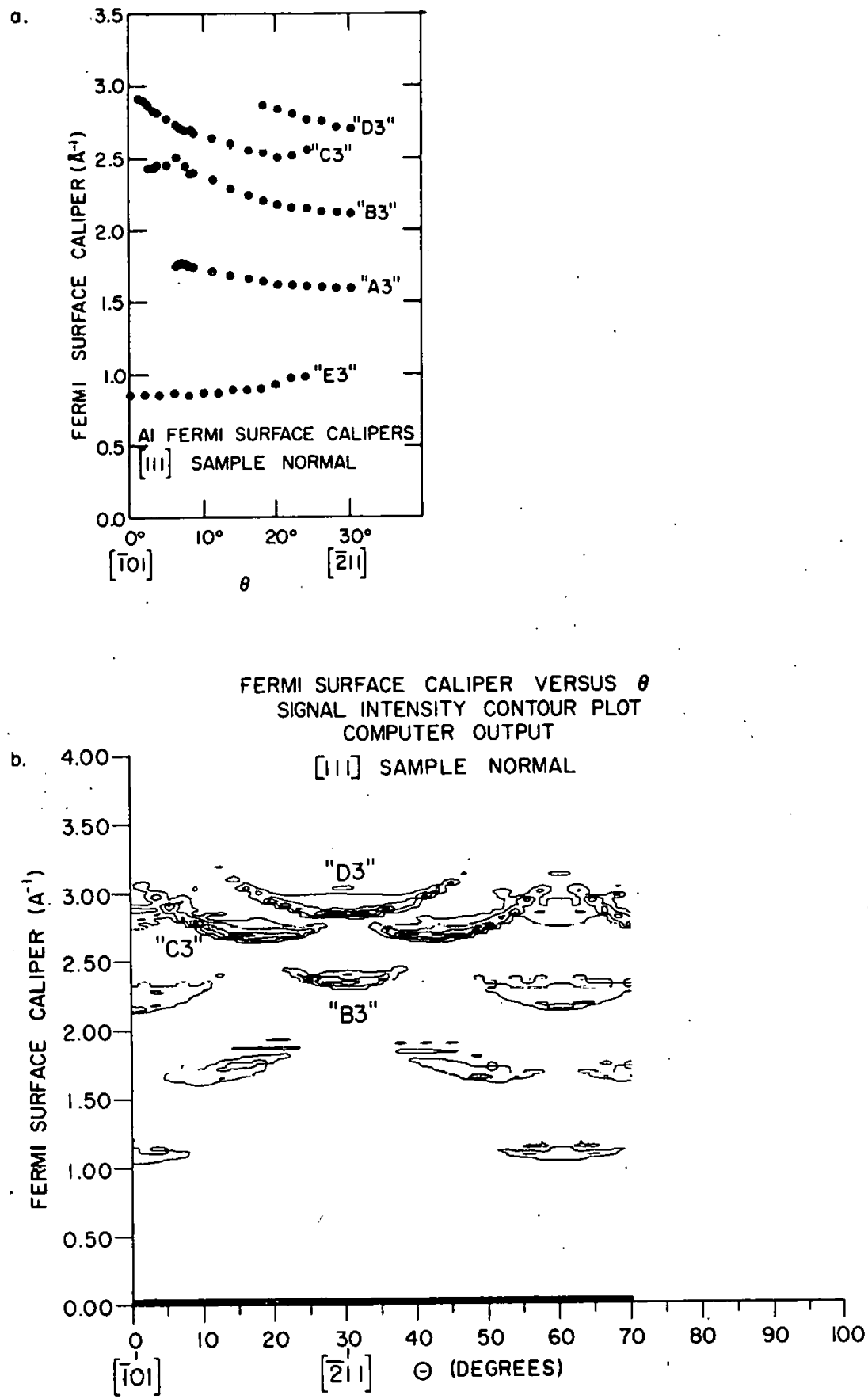

Figure 54. Experimental and theoretical valipers determined for a [111] sample normal 


\section{AL SECOND ZONE FERMI SURFACE CROSS SECTION OVERLAY PLOT COMPUTER OUTPUT}

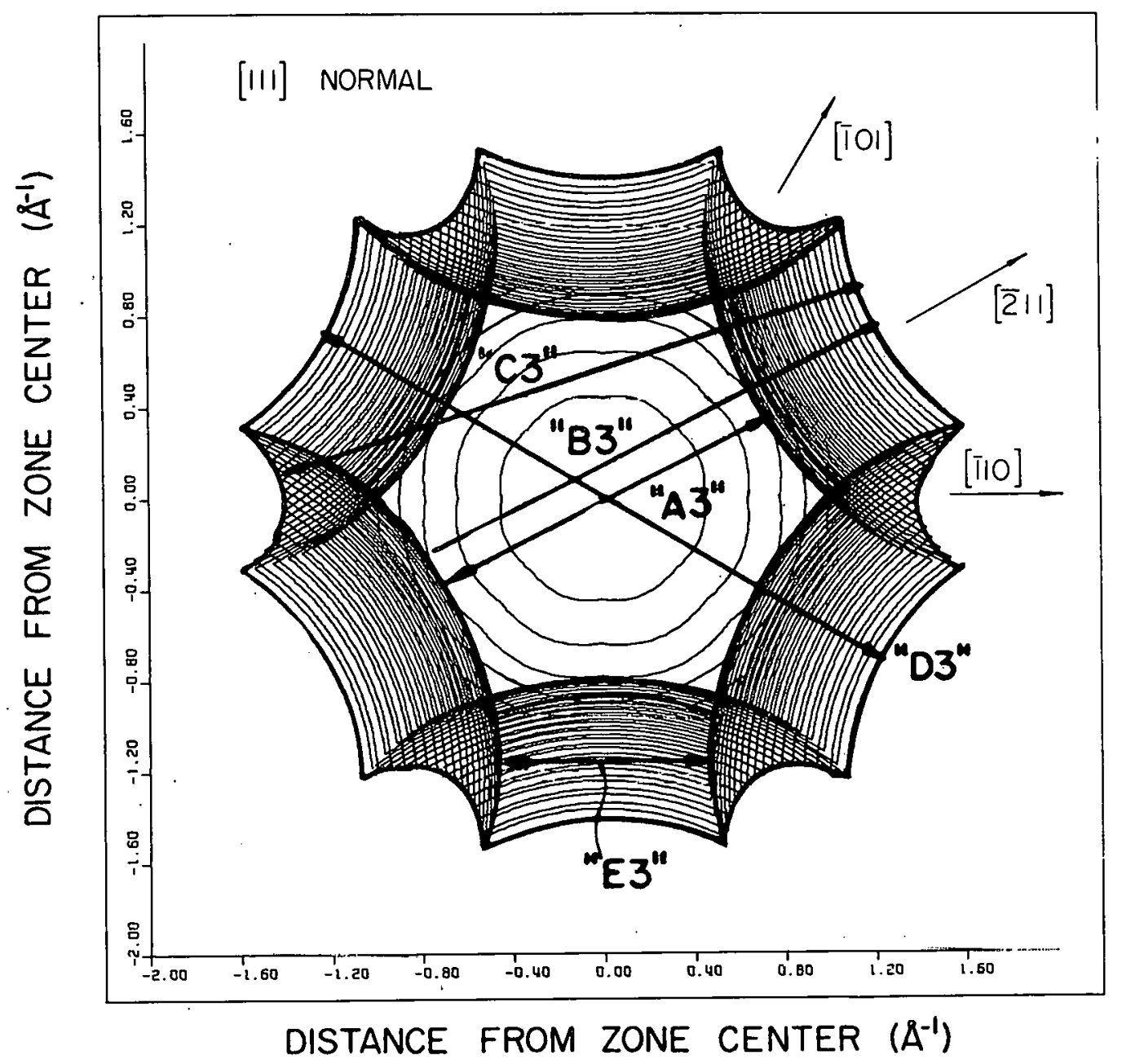

Figure 55. Orbit assignments for experimental zalipers for a [ 111 ] sample normal 
edge but the geodetry involved yields no caliper reduction for the rounding of this edge. The resulting net reduction to the caliper is estimated to be $0.02 \AA^{-1}$. The NFE and adjusted NFE calipers at 300 are then $1.65 \AA^{-1}$ and $1.63 \AA^{-1}$ respectively. The experimental value of $1.59 \AA^{-1}$ is in agreement to within $2 \pi$.

The data also have been compared with the adjusted NFE calipers for this signal at $10^{\circ}$ because both curves are still smoothly varying at $10^{\circ}$. The NFE and adjusted NFE calipers are $1.82 \AA^{-1}$ and $1.808^{-1}$ while the experimental value is 1.728-1. agreenent is within $4 \%$.

For this angular range an inspection of Pigure 55 shows that orbits of type "A3" cross opposing edges of the rS that are almost parallel to each other. Thus many orbits have nearly the same caliper and the signal strength is enhanced. An.examination of the data traces indicates that signal "A3" can be positively identified over the region from $6^{\circ}$ to 300. For less than $6^{\circ}$ the feature broadens and identification is no longer possible. This signal loss could be a direct result of decreasing the number of holes contributing to the signal as the number of orbits of type "a 3 " crossing opposite edges of the hexagonal PS face.

Caliper "B3" in Figure $54 a$ is also due to a partial orbit crossing the hexagonal face of the second-zone fs which is perpendicular to the sample's normal. The orbit crosses 
the hexagonal face and one square face of the second-zone hole surface. It is illustrated in Figure 55. The caliper increases smoothly as the angle changes from $30^{\circ}$ touard 60 were caliper maxima occurs. Examine caliper "B3" in Figure 55. The caliper is seen to reach a maxima at about $6^{\circ}$ as the orbit moves over the point of the FS located between the [ $\overline{2} 11]$ axis and the $[\overline{110}]$ axis. Between $6^{\circ}$ and $30^{\circ}$ the orbit crosses a Type II edge in the same geometry discussed for orbit "A3". so the resulting caliper reduction is estimated to be $0.02 \AA^{-1}$. It also crosses a Type I edge in a manner that ill yield a maximu caliper reduction for that type edge, $0.04 \AA^{-1}$. The net reduction is then $0.06 \AA^{-1}$, but for about $6^{\circ}$ the Type I edge reduction is complicated by a merger with a Type II edge. This corresponds to the rounding encountered at the corner of a square face where a Type II edge intersects two Type I edges. From a graphical method the rounding of this corner as seen at $6^{\circ}$ is expected to be $0.088^{-1}$ and the net reduction will be $0.108^{-1}$. At $30^{\circ}$ the NFE and adjusted NFE calipers are $2.20 \AA^{-1}$ and 2.14\$-1 respectively. The corresponding experimental value is 2.16 1-1 $^{-1}$ resulting in agreement within 1\%. Similarly at 60 the expected values are 2.68\%-1 and 2.58\%-1. The experimental value is $2.50 \AA^{-1}$ and agreement is within $3 \%$. For angles between $6^{\circ}$ and $0^{\circ}$ the orbit crosses the same Type II edge already considered (a reduction of $0.02 \AA^{-1}$ ) and 
another Type II edge that should give almost its full reduction of $0.128^{-1}$. The resulting HFE and adjusted NFB calipers at $0^{\circ}$ are $2.46 \AA^{-1}$ and $2.32 \AA^{-1}$ respectively. The experimental value of $2.428^{-1}$ is taken at $2.5^{\circ}$ due to a lack of signal strength at 00 . agreement is within 4\%. The experimental signal washed out near $0^{\circ}$ and did not result in a caliper value but the caliper is not expected to change significantly in the $2.5^{\circ}$ interval.

Calipers "C3" and "D3" in Figure $54 a$ have been attributed to complete orbits and are expected from the predicted signals of figure $54 \mathrm{~b}$. The orbits contributing to these calipers are indicated on the FS contour overlay plot of Pigure 55. These orbits are of special interest due to the fact that Gantmahker and Krylov (89) did not see similar orbits for their indium sample with a [111] normal axis. They indicated (89, Figure 7) no calipers resulting fron complete orbits for that sample. Orbits similar to nc3n and "D3" should exist for indium as the second-zone hole surface is very similar to that for aluminum. The major difference is the distortion of the indium $\mathrm{Bz}$ as a result of its tetragonal crystal structure.

Caliper "C3" is predicted for angles from 00 to about $25^{\circ}$ (uhere the intensity decreases) with a minima at roughly 180. This caliper will be smaller than predicted due to counding effects, but the reduction will vary with the direc- 
tion of the orbits. For $0^{\circ}$ it crosses two Type I edges and should be reduced by roughly $0.08 \AA^{-1}$. Between $15^{\circ}$ and $25^{\circ}$ the orbit crosses one Type I edge and one Type II edge so the calipers should be reduced by about $0.16 \AA^{-1}$. The resulting NFE and adjusted NFE calipers at $1.3^{\circ}$ are $3.03 \AA^{-1}$ and $2.95 \AA^{-1}$ respectively. The experimental value of $2.91 \%-1$ agrees to within 1\%. The comparison was made at $1.3^{\circ}$ due to the lack of experimental data at $00^{\circ}$. At $20^{\circ}$ the NFE and adjusted NPE calipers are $2.59 \AA^{-1}$ and $2.43 \%-1$ respectively. The experimental value is 2.50 - $^{-1}$ which agrees within $3 \%$.

From Figure 54b it is seen that caliper "D3" is expected to occur for the angular range of $15^{\circ}$ to $30^{\circ}$ but the signals are strongest for angles greater than 200. This orbit crosses two square faces of the second zone fS during its entire range so the caliper reduction is determined by two Type I edges. The geometry involved leads to the expected reduction for each edge being the maximu Type I reduction discussed earlier, $0.04 \mathrm{~A}^{-1}$. Therefore, the net reduction is estimated to be $0.08 \AA^{-1}$. The NFE and adjusted NPB at $20^{\circ}$ are then $2.87 \AA^{-1}$ and $2.79 \AA^{-1}$ respectively. The experimental value of $2.838^{-1}$ agrees to within $1 \%$. At $30^{\circ}$ the three caliper values are $2.78 \AA^{-1}, 2.708^{-1}$, and $2.70 \AA^{-1}$ respective$1 Y$

Caliper "E3" is attributed to a partial orbit across a square face of the second-zone FS. The angular dependence of 
the caliper is shown in Figure $54 a$ and the corresponding hole orbit is indicated on Figure 55. The holes contributing to this signal cross tro Type I edges but the geometry of this case is expected to gield no caliper reduction due to this rounding. The only caliper reduction is expected to occur near $30^{\circ}$ were the effects of the Type II edge counding will round the corners of the square face itself. From a graphical examination this is not expected to affect calipers for angles smaller than $25^{\circ}$, so for the anguiar range of 00 to $24^{\circ}$ there are no corrections to the NPE calipers. At $0^{\circ}$ the NFE and experimental calipers are $0.92 \AA^{-1}$ and $0.86 \AA^{-1}$ respectively. Agreement is within $7 \%$. At $24^{\circ}$ the NPE and experimental values are $1.058-1$ and $0.988^{-1}$ and agreenent is again vithin 7x. The differences seen here in the expected and experimental values can not be removed by simply adjusting the edge rounding. In the geometry involved in this specific case, the calipers are very insensitive to the effects of rounding the edges. The intensity of the signal is expected to decrease near $25^{\circ}$ as the number of electrons that can contribute to the signal decreases as the orbits near the corners on the square face. A corcesponding loss of signal is seen experimentally. This caliper assignment appears to be the only one possible after a thorough examination of the second-zone surface. But the insensitivity of this orbit to rounding effects and the $7 \%$ difference betueen 
observed and expected calipers make it seen unlikely that the assignment is correct.

One additional signal occurs that has not been associated with a hole orbit on the second-zone FS. This signal is seen on Pigures 44 and 45 near 1100 oe at $30^{\circ} \pm 15^{\circ}$. The feature reaches a peak at roughly 1100 oe and $30^{\circ}$. This corresponds to caliper of $1.96 \$-1$ after field corrections have been included. A second-zone orbit has not been identified that would be compatible with the field values or the angular dependence seen in this signal. It is felt that the signal is probably due to a coupling of a third-zone ara orbit and the partial orbit "A3" seen on Pigures $54 a$ and 55. The arm diameter vould then be compatible with estimates made from the de Has-van alphen data of Larson and Gordon (19). The width of the signals for this sample again nakes it very difficult to isolate signals due to different electron orbits. The difficulty in isolating signals from separate orbits in regions like this decreases the chance to resolve regions of this type.

The [111] sample normal calipers are sumarized in Table 6.

\section{Discuㅗ오음}

A comparison of the calipers obtained in this investigation vith previous data is not simply a matter of numerical comparison. As discussed earlier, the RPSE caliper 
Table 6. Caliper results for [111] sample normal

\begin{tabular}{|c|c|c|c|c|c|}
\hline \multirow[t]{3}{*}{ Caliper } & \multirow{3}{*}{$\begin{array}{c}\text { Angle } \\
\text { (Degrees) }\end{array}$} & \multirow{3}{*}{$\begin{array}{c}\text { NFE } \\
\text { caliper } \\
(\mathbb{Q}-1) \\
\text { (1) }\end{array}$} & \multirow{3}{*}{$\begin{array}{c}\text { Adjusted. } \\
\text { RE } \\
(8-1) \\
(2)\end{array}$} & \multirow{3}{*}{$\begin{array}{c}\text { Experimenta } \\
\text { caliper } \\
(8-1) \\
\text { (3) }\end{array}$} & \multirow{3}{*}{$\begin{array}{l}8 \\
\text { difference } \\
\left(\frac{((3)-(2))}{(2)}\right)\end{array}$} \\
\hline & & & & & \\
\hline & & & & & \\
\hline "A 3 " & 0 & 2.13 & 2.05 & - & - \\
\hline nan" & 10 & 1.82 & 1.80 & 1.72 & -4 \\
\hline "A3" & 30. & 1.65 & 1.63 & 1.59 & -2 \\
\hline " B3" & 6. & $2.68^{\circ}$ & 2.62 & 2.50 & -3 \\
\hline " B 3" & 30. & 2.20 & 2.14 & $2: 16$ & +1 \\
\hline "C3n & 1.3 & 3.03 & 2.95 & 2.91 & -1 \\
\hline "C3". & 20. & 2.59 & 2.43 & 2.50 & +3 \\
\hline " D3" & 20 & 2.87 & 2.79 & 2.83 & +1 \\
\hline "E3" & 0 & 0.92 & 0.92 & 0.86 & -7 \\
\hline "E3" & 24 & 1.05 & 1.05 & 0.98 & -7 \\
\hline
\end{tabular}

corresponds to the distances across the shadow of the fS projected on a plane perpendicular to the sample's normal axis. For the second-zone FS of aluminum this means that many calipers do not correspond to a distance across the FS in a single plane perpendicular to the normal axis. Consequently many of the calipers do not correspond to FS dimensions in 1 plane passing through the center of the $\mathrm{BZ}$. 
For this reason the calipers have been compared with calipers derived from the NEE model for the second-zone hole surface. This model has been carefully examined in other studies listed earlier with the only resulting odification being the rounding of sharp edges on the FS. The edge rounding indicated by the studies of Kama and Boha (36) and Pippard (42) have been utilized when comparisons have been made with the NPE FS model. These rounding estinates vere based on the assunption that the rounding of an edge remained the same at every point on that edge. This assumption allowed a phenomenological adjustment to the NFB second-zone calipers.

Before examining the agreement of the present data with the adjusted NEE odel for the second-zone.FS, it is inportant to examine possible sources of experimental ercor in this study.

The accuracy of the experimental FS calipers given in Tables 4, 5, and 6 are lidited by the accuracy of the values of magnetic field and sanple thickness used in Bquation 9. The accuracy of the agnetic field values was subject to two factors. First, for the range of field values used in this study the magnet supply accuracy was limited to \pm 10 oe. Th1s 10 oe uncertainty translates into a caliper uncertainty of $0.02 \AA^{-1}$ to $0.03 \AA^{-1}$ for the thickness ranging from 120 to 196 microns. Thus for the calipers shown in Tables 4, 5, and 
6 this uncertainty ranges from less than 1x to greater than 3\% depending on the sample thickness and the aganitude of the observed calipers.

The second factor of magnetic field uncertainty was related to the accuracy of the selected value of the magnetic field associated ith the onset of the RPSE resonance. When visually selecting the point on a curve where it deriates from the background curve, a finite amount of deviation is needed for detection. This tends to cause field values to be too large. This could contribute to a systematic ercor in the caliper values by making then, too large. This factor was small for sharp features on a RFSE data trace but relatively large for very broad signals. It has already been noted that the data recorded for the [110] sample normal were very veak and it was not possible to determine the onset of the RFSE signal. Instead the first extrema of the resonance was measured for calipers "A2", "B2", and "C2". From the shapes of the data traces it is felt that the onset of the resonance would correspond to calipers smaller than those given in Table 5 by $0.09 \AA^{-1}$ to $0.12 \AA^{-1}$. This correction vould decrease the diffarences seen between the experimental and adjusted NPE caliper values.

signals used to determine the calipers for [100] and [111] normal samples vere much stronger and this problew vas not of major importance. These (df/dH) versus $\mathrm{A}$ traces vere 
recorded twice for each angle to check the reliability of the data and the resulting uncertainty in the calipers is felt to be less than $\pm 0.02 \%-1$ due to mismeasurement of signal

features.

The value of the sample thickness used in Equation 9 resulted from a series of values recorded on a square grid pattern for each sample. For a sample with non-aniform thickness it is very unlikely that a measurement point would coincide with the thickest spot of the sample. In regions not adjacent to the sample edges the changes in thickness occur slowly so it is felt that this could change the thickness by 2 microns. Such a difference would increase the calculated calipers by $1.1 \%$ for the [100] sample, 1.0\% for the [110] sample, and $1.7 \%$ for the [111] sample. yith this must be included the uncertainty of the recorded values taken with the microscope of \pm 2 microns. The net effect of these uncertainties on the caliper error bars is for the [ 100 ] sample $-1.1 \%$ to $+2.2 \%$, for the $[110]$ sample $-1.0 \%$ to $+2.0 \%$. and for the $[111]$ saple $-1.7 x$ to $+3.4 x$.

It should also be noted that the NFE calipers as contained in Figures 24,25 , and 26 introduce sone uncertainty as the values have been grouped in intervals that are $0.03125 \AA^{-1}$ wide. Thus the caliper value assumed for a given interval may differ from the actual value by $\pm 0.03 \&^{-1}$. This must be considered when the agreement between the adjusted 
NFE and the experimental calipers is examined.

The experimental calipers as seen in Figures $49 a, 52 a$, and 54 a exhibit very little scatter from point to point. The uncertainties mentioned above are thus related to the calibration of all the caliper values and not the uncertainty in the calipers as seen from point to point (i.e. systematic errors).

The experimeatal and adjusted NFE second-zone calipers are compatible when error bars are evaluated for the two sets of calipers. For the [110] normal sample it was also necessary to estimate the caliper reduction associated with finding the onset of the BPSE signal. The FS edge rounding estimates used have lead to consistent agreement between the two sets of calipers. The current data indicate that no changes in the adjusted NFE second-zone FS need be considered to obtain the observed angular dependence and magnitude for the PS calipers. 
VII. COHCLUSIONS

The Fermi surface of aluminum has been studied by the BFSE technique using samples with nornai axes along the three major symuetry axes. The resulting FS calipers have been examined and many have been identified with specific hole orbits on the second-zone hole surface. The caliper magnitudes and angular dependence observed are in good agreement with the MPE second-zone.FS when FS edge rounding is taken into account.

In the course of this investigation the use of frequency studies was examined as a useful tool to determine the resonant field values for recorded data. The method employed by cleveland (51). was examined in detail and found to yield the proper results only for perfectly flat samples. Nonuniformity in sample thickness was found to cause the resulting field value and exponent of frequency dependence to be artificially low. This was experimentally substantiated.

The effects of non-uniform sample thickness were also observed on the signals recorded in the RFSE. It was seen that a wedge-shaped sample resulted in signal splitting vhile samples uith a non-unifora but randon thickness give rise to signal broadening and signal distortion. 


\section{LITERATORE CITED}

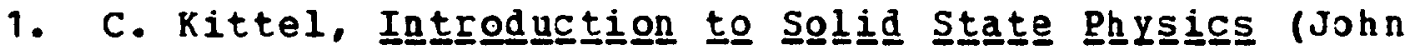
Wiley and Sons, Inc.. New York, 1953).

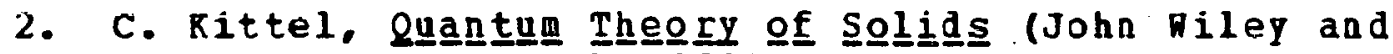
Sons, Inc., New York, 1963).

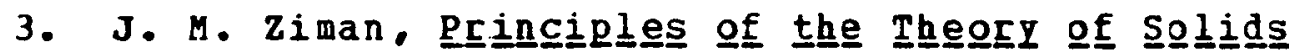
(Cambidge University Press, Cambridge, England, 1965).

4. L. P. Bouchaert, R. Smoluchowski, and E. Wigner, Phys. Rev. 드, 58 (1936).

5. E. M. Gunnersen, Phil. Trans. Roy. Soc. (London) A249, 299 (1957).

6. V. Heine, Proc. RoY. Soc. (London) A24ㅡ. 340 (1957).

7. V. Heine, Proc. Roy. Soc. (London) A2농. 354 (1957).

8. V. Heine, Proc. Roy. Soc. (London) 소농, 361 (1957).

9. T. E. Faber and A. B. Pippard, Proc. Roy. Soc. (London) A231, 336 (1955).

10. D. H. Howling, E. Mondoza, and J. E. Zimmerman, Proc. Roy. Soc. (London) A2 $2 \underline{2}$. 86 (1955).

11. E. Pawcett, Phys. Rev. Letters 3 . 139 (1959).

12. D. N. Langenberg and T. N. Moore, Phys. Rer. Letters $\underline{3}$, 137 (1959).

13. A. A. Harrison, Phys. Rev. 11ㅁ. 555 (1959).

14. H. A. Harrison, Phys. Rev. 118, 1182 (1960).

15. T. M. Moore and F. W. Spong. Phys. Rev. 12ㄷ, 846 (1962).

16. B. Segal, Phys. Rev. 12노, 1797 (1.961).

17. M. G. Priestley, Phil. Mag. I, 1205 (1962).

18. N. H. Ashcroft, Phil. Hag. g, 2055 (1963) .

19. C. O. Larson and h. L. Gordon, Phys. Rev. 15‥ 703 (1967). 
20. N. A. Harrison, Phys. Rer. 118, 1190 (1960).

21. B. Segal, Phys. Rev. 131, 121 (1963).

22. E. C. Snow, Phys. Rev. 158, 683 (1967).

23. F. C. Greisen, Phys. St atus Solidi 25. 753 (1968).

24. J. H. D. Connol1y. Bull. An. Phys. Soc. 14. 359 (1969).

25. J. S. Paulkner, Phys. Rev. 178, 914 (1969).

26. V. Hoffstein and D. S. Boudreaux, Phys. Rev. B2, 3013 $(1970)$.

27. J. R. Anderson and S. S. Lane, Phys. Rev. B 2, 298 (1970).

28. J. P. G. Shepherd, C。 O. Larson, D. Roberts, and R. L.

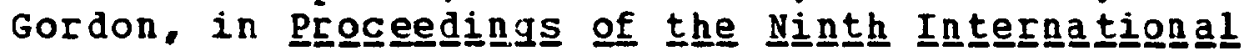

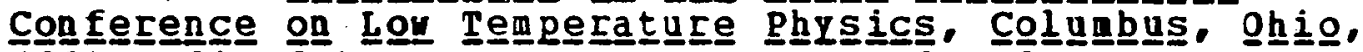
1964, edited by J. G. Daunt, D. V. Bdwards, F. J. Milford, and M. Yaqub (Plenum Press, Inc.. New York, 1965). p. 752 .

29. A. A. Galkin, V. P. Naberezhnykh, and V. A. Mel'nik, Zh. Eksperim i Teor. Fiz. $4 \underline{4} 127$ (1963) iSov. PhYs.JETP 17. 87 (1963) ].

30. V. P. Naberezhnykh and V. P. Tolstoluzhskii, Zh. Eksperim i Teor. Fiz. 46, 18 (1964) [Sov. Phys.-JETP 19. 13 (1964) ].

31. F. D. Spong ayd. A. F. Kip, Phys, Rev. 137, A431 (1965).

32. B. M. Roberts, Phys, Rev, 119, 1889 (1960).

33. A. A. Bezuglyi, A. A. Galkin, and A. I, Pushkin, zh. Eksperim i Teor. Fiz. 4 4. 71 (1963) [Sov. Phys.-JETP 1]. 50 (1963) ].

34. B. R. Jones, Phil. Mag. 9, 217 (1964).

35. K. Fossheim and T. Olsen, Phys. Statas Solidi 6.867 (1964).

36. G. N. Kamm and H. V. Bohm. Phys. Rev. 131, 111 (1963).

37. R. Stedman and G. Nilsson, Phys. Rev. Letters 15, 634 (1965). 
38. J. M. Meymouth and R. Stedman, PhyS. Rev. B 2, 4743 (1970) •

39. R. J. Balcombe, Proc. Roy. Soz. (London) A2 I5, 113 (1963) •

40. K. Porsvoll and I. Holwech, Phil: Mag. 9, 435 (1964).

41. E. S. Borovik and 7. G. Volotskaya, Zh. Eksperim i Teor. Fiz: 48, 1554 (1965) [SOV:Phys.-JETP 21, 1041 (1965) ]:

42. A. B. Pippard, Proc. Roy. Soc. (London) A2 26․, 464 $(1964)$.

43. R. J. Balcombe and R. A. Parker, Phil. llag. 21. 533 (1970) .

44. G. Brandli, P. Cotti, E. M. Fryer, and J. L. Olsen, in

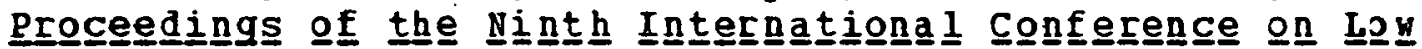

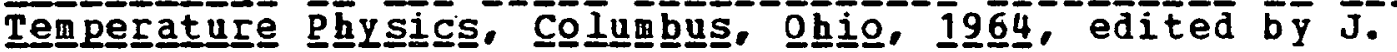
G. Daunt, D. V. Eduards, F. J. Milford, and M. Yaqub (Plenum Press, Inc., Ne York, 1965), p. 827.

45. I. Holwech and R. Bisnes, Phil. Mag. 17, 757 (1968).

46. R. Risnes, Phil. Hag. 21, 591 (1970).

47. E. P. Vol'skii, Zh. Eksperia i Teor. Fiz. 4․6, 123 (1964) [SOF. Phys.-JETP 19.89 (1964)].

48. S. Balibar, B. Perrin, and A. Libchaber, J. Phys. F (Metal Physics) 2. 629 (1972).

49. V. F. Gantmakher, Zh. Eksperim i Teor. Fiz. 4ㄴ., 1416 (1962) [SOV. Phys.-JETP 15. 982. (1962)].

50. I. P. Krylov and V. F. Gantmakher, Zh. Eksperim i Teor. Fiz. 51, 740 (1966) [SOV. Phys.-JETP 24. 492 (1967)].

51. J. R. Cleveland, Ph.D. Thesis, Physics Department, Iowa State University, 1970 (unpublished).

52. B. Perrin, G. Heisbuch, and A. Libchaber, Phys. Rev. B1. 1501 (1970) .

53. A. Fukumoto and M. H. P. Strandberg, Phys. Rev. 15 $\underline{5}$, 685 (1967). 
54. J. R. Cleveland and J. L. Stanford, Phys. Rev. B互 311 (1971).

55. V. V. Boiko and V. A. Gasparov, Zh. Eksperim i Teor. Piz. 61. 1976 (1971) [SOV. Phys.-JETP 3ㄴ. 1054 (1972) ].

56. M. M. P. Matthey and A. R. Devroomen, Phys. Letters 36A. $59(1971)$.

57. E. A. Kaner, Physics 3., 285 (1967).

58. G. E. Juras, Phys. Rev. 18I, 784 (1969).

59. E. S. Takle, Ph.D. Thesis, Physics Department, Iowa State Oniversity, 1971 (unpublished).

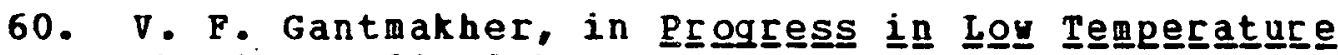
Physics. edited by C. J. Gorter (North-Holland Publishing Company, Amsterdam, The Netherlands, 1965). Vol. V, p. 181 .

61. E. A. Kaner and V. F. Gantmakher, Osp. Fiz. Nauk 94. 193 (1968) [Sov. Phys.-Uspekhi 11, 81 (1968)].

62. M. M. Malsh, Jr., in soliid state Physiçs, edited by J. F. Cochran and R. R. Haering (Gordon and Breach Science Publishers, Inc.. Ney York, 1968), Vol. I, p. 127.

63. J. F. Koch and T. K. Magner, Bull. At: Soc, 11, 170 $(1966)$.

64. R. A. Herrod, C.A. Gage, and R. G. Goodrich, Phys. Rev. BL. 1033 (1971).

65. V. F. Gantmakher and V. T. Dolgopolov, Zh. Eksperim i Teor. Fiz. 60, 2260 (1971) [Snv. Phys.-JETP 33, 1215 (1971) ].

66. V. F. Gantmakher, Zh. Eksperim i Teor. Fiz. Pis'ma'v Redakt. 2. 557 (1965) [Sov. Phys.-JETP Letters 2. 346 (1965) ].

67. V. F. Gantmakher and Yu. S. Leonov, Zh. Eksperim i Teor. Fiz. Pis'ma $v$ Redakt. 8,264 (1968) [Sov. Phys.-JETP Letters $\underline{8}, 162$ (1968)].

68. V. P. Naberezhynkh and A. A. Mar'yakhin, Phys. Status Solidi 2ㅁ. 737 (1967). 
69. R. C. Jones, R. G. Goodrich, and L. M. Palicov, Phys. Rev. 174. 672 (1968).

70. V. P. Naberezhnykh, A. A. Mar'yakhin, and V. L. Mel'nik, Zh. Eksperim i Teor. Fiz. ${ }_{2}$. 617 (1967) [Sov. Phys.-JETP 25, 403 (1967) ].

71. R. G. Goodrich and R. C. Jones, Phys: Rev. 156. 745 (1967) .

7.2. D. A. Boudreau and R. G. Goodrich, PhYS. Rev. B $\underline{3}, 3086$ (1971).

73. V..P. Naberezhngkh and D. T. Tsymbal, Zh. Eksperia i Teor. Fiz. 60. 259 (1971) [Sov. Phys.-JETP $\underline{3}, 142$ (1971) ].

74. S. B. Soffer, R. Huguen in, and P. A. Probst, Journal of Lov Temperature Physics 11, 537 (1973).

75. V. P. Naberezhnykh and D. T. Tsymbal, Zh. Eksperim. i. Teor. Fiz. Pis'ma $v$ Redakt. 5,319 (1967) [ Sov.

Phys.-JETP Letters $5,263(1967)$ ].

76. A. Myers, S. G. Porter, and R. S. Thompson, J. Phys. F (Metal Physics) 2,24 (1972).

77. J. P. D'Haenens, A. Libchaber, C. LaRoche, and J. LeHericy, Phys. Letters 2 읏․ 312 (1968) :

78. B. Perrin and A. Libchaber, in progeceedings of $\underline{\text { the }}$ e Eleventh Internation physics, st. Andreus, scot 1 and. 196, edited by J. F. Allen. D. I. Inlayson, and M. HCCall (University of St. Andrews, St. Andrews, Scotland, 1968), p. 1191.

79. D. M. Sparlin and D. S. Schreiber, in proceedings of

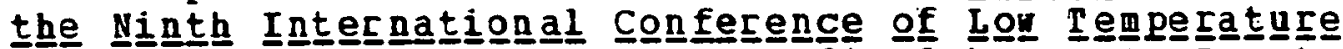

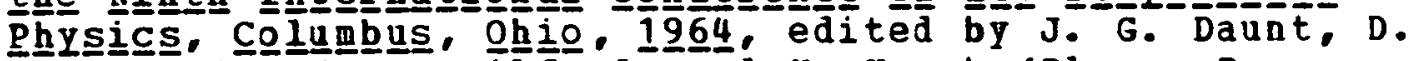
V. Edvards, F. J. Milford, and M. Yaqub (Plenum Press, Inc.. New York, 1965), p. 823 .

80. S. Foner and E. T. McNiff, Jr., Rev. Sci. Instr. 38, 931 (1967).

81. P. H. Haberland C. A. Shiffman, Phys. Rev. Letters 19, 1337 (1967).

82. P. H. Haberland, J. F. Cochran, and C. A. Shiffman, Phys. Rev. 184. 655 (1969). 
83. A. A. Mar'y akhin and V. P. Naberezhnykh, Zh. Eksperim. i Teor. Fiz. Pis'ma Redakt. 3, 205 (1966) [Sov. Phps.-JETP Letters 3,130 (1966) ].

84. J. F. Cochran and C. A. Shiffman, Phys. Rev. 1440, A 1679 (1965).

85. P. H. Haberland, H.S. Thesis, Department of Physics, MIT 1966 (unpublished).

86. A. Fukunoto and M. M. P. Strandberg. Phys. Letters $2 \underline{3}$. 200 (1966).

87. P. H. Haberland, J. F. Cochran, and C. A. Shiffman, Phys. Letters 3 으, 476 (1969).

88. V. F. Gantmakher and I. P. Rrylov. Zh. Eksperia i Teor. Fiz. 4‥ 2111 (1964) [Sor. PhYs.-JETP 2ㅇ. 1418 (1965)].

89. V. F. Gantmakher and I. P. Rrylov, Zh. Eksperim i Teor. Fiz. 49, 1054 (1965) [SOr. Phys.-JETP 22, 734 (1966)]:

90. I. P. Krylov, Zh. Eksperim i Teor. Piz. 5ㄴ. 1738 (1968) [SOv. Phys.-JETP 2]. 934 (1968)].

91. V. S. Tsoi, Zh. Eksperim. i. Teor. Fiz. Pis'ma Redakt. 15. 246 (1972) [Sov. Phys.-JETP Letters 1‥ 171 (1972)].

92. I. P. Krylov, Zh. Eksperim i Teor. Fiz. Pis'ma v Redakt. 1, $116(1965)$ [ Sov. Phys.-JETP Letters 1, 116 (1965) ].

93. P. M. Snyder, J. Phys. P (Metal Physics) 1. 363 (1971).

94. P. Roach, Phys. Rev. B出 1748 (1971).

95. V. V. Boiko, V. A. Gasparov, and I. G. Grerdtsiteli, Zh. Eksperia $i$ Teor. Fiz. 트, 489 (1969) [Sov. PhYS.-JETP 29, 267 (1969) ].

96. J. R. cleveland and J. L. Stanford, Phys. Rer. Letters 24. 1482 (1970).

97. V. V. Boiko, V. A. Gasparov, and I. G. Grerdtsiteli, Zh. Eksperim i Teor. Fiz. Pis'ma v Redakt. 6, 737 (1967) [Sov. Phys.-JETP Letters 6, 212 (1967)].

98. J. F. Koch and T. K. Magner, Phys. Rev. 151, 467 (1966). 
99. P. S. Peercy, R. M. Malsh, Jr., L. R. Rupp, Jr., and P.

H. Schmidt, Phys. Rev. 171. 713 (1968).

100. T. K. Ragner and J. F. Koch, Phys. Rev. 165.885 (1968).

101. T. G. Blaney, Phil. Mag. 2ㅁ, 23 (1969).

102. A. Libchaber, G. Adams, and C. C. Grimes, Phys. Rev. B1, 361 (1970).

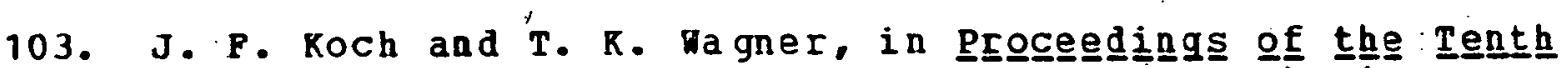

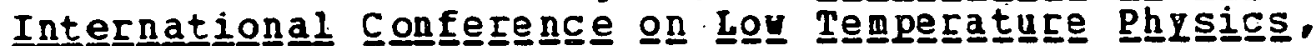
Mosc으. 1966, edited by M. P. Ma1kov (ProizvodstrennoIzdatel'skii kombinat, VINITI, loscow, $1967)$. p. 124 .

104. P. N. Trofimenkoff, Solid State Communications 11. 743 (1972).

105. T. G. Blaney and D. Parsons, J: Phys. C (Solid state) $\underline{3}$. 126 (1970).

106. V. S. Tsoi and V. F. Gantmakher, 2 h. Eksperim i Teor. Fiz. 56, 1232 (1969) [Sov. Phys.-JETP 29, 663 (1969)].

107. P. S. Peercy and M. Malsh, Jr., PhYs. Rev. Letters 17. $741(1966)$.

108. J. 0. Henningsen, Solid state Communications 2, 763 (1969):

109. J. E. Bradfield and J. B. Coon, 1970 Harch Heeting of The American Physical society, Solid state Division. Dallas. Texas, 23-26 March 1970. Abstracted in Bull. An Phys. Soc. 15. 295 (1970).

110. J. E. Bradfield, M. S. Thesis, Department of Physics, University of Houston. 1970 (unpublished).

111. C. A. Gage and R. G. Goodrich, 1970 March Heeting of The American Physical Society. Solid State Division. Dallas, Texas, 23-26 March 1970. Abstracted in Bull. An. Phys. SOC. 15, $295(1970)$.

112. C. A. Gage and R. G. Goodrich, Phys. Rev. B 3,3214 (1971) . 
113. E. S. Takle and J. L. Stanford, J. Phys. P (Metal Physics) 3, 1956 (1973).

114. J. E. Bradfield and J. B. Coon, Phys. Rev. BI, 5072 (1973) .

115. V. F. Gantmakher and E. A. Kaner, Zh. Eksperim i Teor. Fiz. 4ㅇ․ 1572 (1965) [Sov. Phys.-JETP 21, 1053 (1965)].

116. v. F. Gantmakher, Zh. Eksperim i Teor. Fiz, 44. 811 (1963) [SOV. Phys.-JETP 17. 549 (1963)].

117. V. F. Gantmakher and E. A. Kaner, Zh. Eksperim i. Teor. F.12. 4., $1430(1963)$ [S Ov. PhYS.-J.ETP 18, 988 (1964)].

118. V. F. Gantmakher, zh. Eksperim i.Teor. Fiz. 4느, 345 (1962) [SOV. PhYS.-JETP 16, 247 (1962)].

119. M. M. M. P. Matthey and A. R. Devroonen, Solid state Commanications 9.1329 (1971).

120. V. F. Gantmakher and Yu. V. Sharvin, Zh. Eksperim i Teor. Fiz. 48. 1077 (1965) [SOv. Phys.-JETP 21, 720 (1965) ].

121. V. F. Gantmakher, 2h. Eksperim i Teor. Piz. 4ㅌ, 2028 (1964): [SOV. Phys.-JETP 19. 1366 (1964)].

122. H. H. A. Awater and J. S. Lass, J. Phys. F (Hetal Physics) 3 , 1113 (1973).

123. H. F. Druyvesteyn and A.J. Smets, Journal of Low Temperature Physics 2, 619 (1970).

124. M. M. M. R. Matthey, M. A. C. Devillers, and A. R. DeVroomen. Phys. Status Solidi (b) 63. 279 (1974).

125. M. A. C. Devillers, H. M. M. P. Matthey, and A. R. DeVroomen, Phys. Status Solidi (b) 63. 471 (1974).

126. G. P. Hughes and J. R. Merrill, Phys. Rev. Bg, 2423 (1974).

12\%. W. F. Druyvesteyn and A.J. Smets, Phys. Letters 30 A. 415 (1969). 
128. M. M. Walsh, Jr., C. C. Grimes, G. Adams, and L. H. Rupp. Jr.. in proceedings of the Ninth Intergratiogional

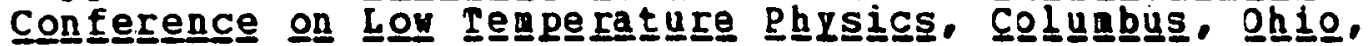
1964, edited by J. G. Daunt, D. V. Bdwards, F. J. Milford, and M. Yaqub (Plenua Press, Inc., New York, 1965), p. 765 .

129. V. V. Boiko and V. A. Gasparov, Zh. Eksperim i Teor. Piz. 61, 2362 (1972) [SOV. Phys.-JETP 3ㄴ., 1266 (1973)].

130. R. S. Thompson and A. Myers, J. Phys. F (Metal Physics) 3. L64, (1973).

131. O. L. Steenhaut and R. G. Goodrich, Phys. Rev. B1. 4511 $(1970)$.

132. E. A. Kaner, Zh. Eksperim i Teor. Fiz. 44, 1036 (1963) [Sov. Phys. -JETP 17. 700 (1963) ].

133. G. E. Juras, Phys. Re . Letters 24. 390 (1970):

134. G. E. Juras, Phys. Rev. B2, 2869 (1970).

135. E. A. Kaner and V. L. Fal'ko, Zh. Eksperim i Teor. Piz. 51. 586 (1966) [SOV. Phys.-JETP 24. 392 (1967)].

136. G. Meisz, Phys. Rev. 149, 504 (1966).

137. R. G. Chambers, Phys. kondens. Haterie 9, 171 (1969) .

138. S. Soffer, J. Low Temperature Physics 9, 551 (1972).

139. E. A. Kaner and V. L. Fal'ko, Zh. Eksperim i Teor. Fiz. 49. 1895 (1965) [Sov. Phys.-JETP 22, 1294 (1966)].

140. J. A. Hoekstra and J. L. Stanford, Phys. Bev. B, 1416 (19.73).

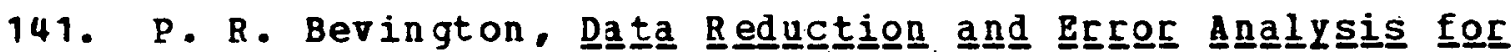
the Phrsical sciences (McGrau-Hili Book Company, New York, 1969).

142. R. R. Symon, Mechanies (Addison-Mesley Pablishing Company, Reading, Massachusetts, 1960).

143. P. S. Peercy, U, M. Halsh, Jr., I. M. Rupp, Jr., and P. H. Schmidt, PhYS. Rev. 171, 713 (1968). 
144. V. F. Gantmahker and I. P. Krylor, Zh. Eksperim i Teor. Piz. 49, 1054 (1965) [Sov. Phys.-JETP 2.2. 734 (1966)].

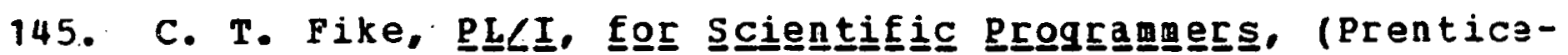
Hal1, Inc., Bnglewood Cliffs, New Jersey. 1970).

146. G. D. Hanchett, Radio Corporation of Anerica publication number ST-3520, 1967.

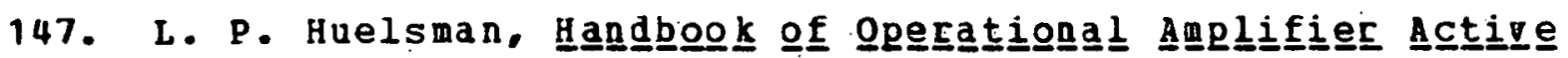
RC Netyorks, (Burr-Brorn Research Corporation, Tucson, Arizona, 1966).

148. S. H. Hui and J. A. Rayne, Journal of Low Temperature Physics 10.635 (1973).

149. F. E. Metz, Ph.D. Thesis, Chemistry Department, Iowa State University, 1960 (unpublished). 
IX. AC RNONLEDGMENTS

I wish to thank professor John L. Stanford for suggesting the topic of this investigation and for his guidance and encouragement during the course of the investigation.

I wish to express my sincere appreciation to Mr. Mark Dakins for his extensive efforts during the formulation and coding of the caliper computer program. His assistance and guidance were instrumental in witing a program with a general usefulness for this calculation.

Timely discussions with Dr. Robert Powell and Dr. Michael Lind have provided valuable ideas and encouragement for solving several technical problems encountered during this study. For their effort and concern I am very grateful. I wish also to express my appreciation to Messrs. Richard Brown, Edward Sexhaner, and George Holland for their advice in preparing mechanical and electrical apparatus used in this study.

To my wife Mary I want to express special appreciation for her encouragement and patience during the course of this investigation and for typing the manuscript.

The financial assistance of the Department of Health, Education, and gelfare in the forn of a NDEA ritle IV Fellowship during part of this investigation is certainly appreciated. 